\title{
ENTWINED MODULES \\ OVER LINEAR CATEGORIES AND GALOIS EXTENSIONS
}

\author{
BY \\ Mamta Balodi, ${ }^{*}$ AbhisheK BanerJeE ${ }^{* *}$ And SAmarpita Ray \\ Department of Mathematics, Indian Institute of Science, Bangalore - 560012, India \\ e-mail: mamta.balodi@gmail.com,abhishekbanerjee1313@gmail.com, \\ ray.samarpita31@gmail.com
}

ABSTRACT

In this paper, we study modules over quotient spaces of certain categorified fiber bundles. These are understood as modules over entwining structures involving a small $K$-linear category $\mathcal{D}$ and a $K$-coalgebra $C$. We obtain Frobenius and separability conditions for functors on entwined modules. We also introduce the notion of a $C$-Galois extension $\mathcal{E} \subseteq \mathcal{D}$ of categories. Under suitable conditions, we show that entwined modules over a $C$-Galois extension may be described as modules over the subcategory $\mathcal{E}$ of $C$-coinvariants of $\mathcal{D}$.

\section{Introduction}

The purpose of this paper is to study a theory of modules over quotient spaces of certain categorified fiber bundles. Suppose that $X$ is an affine scheme over a field $K$ and let $G$ be an affine algebraic group scheme with a free action $\sigma: X \times G \longrightarrow X$ on $X$. Let $Y$ be the quotient given by the coequalizer

$$
X \times G \underset{p r}{\stackrel{\sigma}{\longrightarrow}} X \stackrel{p}{\longrightarrow} Y .
$$

If $X \longrightarrow Y$ is faithfully flat and the canonical map can : $X \times G \longrightarrow X \times_{Y} X$ is an isomorphism, then $X$ is said to be (see, for instance, [24], [28]) a principal fiber bundle over $Y$ with group $G$.

* M.B. was also supported by SERB NPDF grant PDF/2017/000229.

** A.B. was also supported by SERB Matrics fellowship MTR/2017/000112.

Received June 21, 2019 
The algebraic counterpart of (1.1) consists of an algebra $A$, a Hopf algebra $H$ and a coaction $\rho: A \longrightarrow A \otimes H$ that makes $A$ into a right $H$-comodule algebra. Let

$$
B:=A^{c o H}=\left\{a \in A \mid \rho(a)=a \otimes 1_{H}\right\}
$$

be the algebra of coinvariants of $A$, i.e., $B$ is given by the equalizer

$$
B \longrightarrow A \underset{\text { in }}{\stackrel{\rho}{\longrightarrow}} A \otimes H \text {. }
$$

In this case, there is a canonical map can : $A \otimes_{B} A \longrightarrow A \otimes H$ determined by setting $\operatorname{can}(x \otimes y)=x \cdot \rho(y)$. If the Hopf algebra $H$ has bijective antipode, $B \longrightarrow A$ is a faithfully flat extension and can : $A \otimes_{B} A \longrightarrow A \otimes H$ is an isomorphism, it was shown by Schneider [28] that modules over $B$ may be recovered as the category of " $(A, H)$-Hopf modules."

We work with a small $K$-linear category $\mathcal{D}$, a $K$-coalgebra $C$ and an "entwining structure" $\psi$ consisting of a collection of morphisms

$$
\psi=\left\{\psi_{X Y}: C \otimes \operatorname{Hom}_{\mathcal{D}}(X, Y) \longrightarrow \operatorname{Hom}_{\mathcal{D}}(X, Y) \otimes C\right\}_{(X, Y) \in \mathrm{Ob}(\mathcal{D})^{2}}
$$

satisfying conditions that we lay out in Section 2. We consider the category $\mathscr{M}(\psi)_{\mathcal{D}}^{C}$ of modules over the entwining structure $(\mathcal{D}, C, \psi)$ (see Definition 2.2). These may be seen as modules over a "categorical quotient space" of $\mathcal{D}$ with respect to the coalgebra $C$ and the entwining $\psi$.

The notion of a $C$-Galois extension $\mathcal{E} \subseteq \mathcal{D}$ of categories is introduced in Section 4. Additionally, a $C$-Galois extension gives rise to a canonical entwining structure on $\mathcal{D}$. Under certain conditions, we show that modules over the category $\mathcal{E}$ of $C$-coinvariants of $\mathcal{D}$ may be described as modules over the canonical entwining structure.

Entwining structures for algebras were introduced by Brzeziński and Majid in [7] and it was realized in Brzeziński [3] that entwined modules provide a unifying formalism for studying diverse concepts such as relative Hopf modules, Doi-Hopf and Yetter-Drinfeld modules as well as coalgebra Galois extensions. In fact, the study of entwining structures for algebras and entwined modules over them is well developed in the literature and we refer the reader, for instance, to [1], [3] [5], [9], [10], [12], [21], [27] for more on this subject.

Our notion of modules over an entwining structure $(\mathcal{D}, C, \psi)$ builds on the analogy of Mitchell [22] which says that a small $K$-linear category should be seen as a " $K$-algebra with several objects." In particular, the category $\mathscr{M}(\psi)_{\mathcal{D}}^{C}$ 
also generalizes the "relative $(\mathcal{D}, H)$-Hopf modules" studied in our previous work in [2], where $H$ is a Hopf algebra and $\mathcal{D}$ is an $H$-comodule category in the sense of Cibils and Solotar [16]. In other words, $\mathcal{D}$ is a small $K$-linear category whose morphism spaces are equipped with a coaction of $H$ that is compatible with composition. When $\mathcal{D}$ has a single object, it reduces to an ordinary $\mathrm{H}$ comodule algebra and the relative $(\mathcal{D}, H)$-Hopf modules reduce to the usual notion of relative Hopf modules (see Takeuchi [30]).

For Doi-Hopf modules, Frobenius and separability conditions were studied extensively in a series of papers [13], [14], [15]. Later, Brzeziński studied Frobenius and Maschke type theorems for entwined modules in [4]. In this paper, we proceed in a manner analogous to the unified approach of Brzeziński, Caenepeel, Militaru and Zhu [8] for studying Frobenius and separability conditions for entwined modules over $(\mathcal{D}, C, \psi)$.

The idea is as follows: the "categorical quotient space" of $\mathcal{D}$ with respect to $C$ and $\psi$ may be thought of as a subcategory of $\mathcal{D}$ and $\mathscr{M}(\psi)_{\mathcal{D}}^{C}$ plays the role of modules over this subcategory. Although this "subcategory" of $\mathcal{D}$ need not exist in an explicit sense, we would like to study the properties of this extension of categories. In particular, we would like to know if it behaves like a separable, split or Frobenius extension of small $K$-linear categories. For this, we turn to a pair of functors

$$
\mathscr{F}: \mathscr{M}(\psi)_{\mathcal{D}}^{C} \longrightarrow \operatorname{Mod}-\mathcal{D}, \quad \mathscr{G}: \operatorname{Mod}-\mathcal{D} \longrightarrow \mathscr{M}(\psi)_{\mathcal{D}}^{C}
$$

Here $\mathscr{F}$ is the left adjoint and behaves like an "extension of scalars" whereas its right adjoint $\mathscr{G}$ behaves like a "restriction of scalars." We recall here (see [8, Theorem 1.2]) that in the classical case of an extension $R \longrightarrow S$ of rings inducing the pair of adjoint functors $\operatorname{Mod}-R \stackrel{G}{\stackrel{G}{\leftrightarrows}} \operatorname{Mod}-S$ given by extension and restriction of scalars, we have:

$R \longrightarrow S$ is split extension $\Leftrightarrow$ Left adjoint $F: \operatorname{Mod}-R \longrightarrow \operatorname{Mod}-S$ is separable,

$R \longrightarrow S$ is separable extension $\Leftrightarrow$ Right adjoint $G: \operatorname{Mod}-S \longrightarrow \operatorname{Mod}-R$ is separable,

$R \longrightarrow S$ is Frobenius extension $\Leftrightarrow(F, G)$ is Frobenius pair of functors.

It is therefore natural to study criteria for the separability of the functors $\mathscr{F}$ and $\mathscr{G}$ as well as conditions for $(\mathscr{F}, \mathscr{G})$ to be a Frobenius pair of functors. 
In this paper, we will always use the following convention: for $f \in \operatorname{Hom}_{\mathcal{D}}(Y, X)$ and $c \in C$, we write

$$
\psi_{Y X}(c \otimes f)=f_{\psi} \otimes c^{\psi} \in \operatorname{Hom}_{\mathcal{D}}(Y, X) \otimes C
$$

with the summation omitted. We write $h: \mathcal{D}^{o p} \otimes \mathcal{D} \longrightarrow$ Vect $_{K}$ for the canonical $\mathcal{D}$-D-bimodule $h(Y, X)=\operatorname{Hom}_{\mathcal{D}}(Y, X)$. The entwining structure makes $h \otimes C$ into a $\mathcal{D}$ - $\mathcal{D}$-bimodule by setting

$$
(h \otimes C)(Y, X):=\operatorname{Hom}_{\mathcal{D}}(Y, X) \otimes C, \quad((h \otimes C)(\phi))(f \otimes c):=\phi^{\prime \prime} f \phi_{\psi}^{\prime} \otimes c^{\psi},
$$

for any $(Y, X) \in \mathrm{Ob}\left(\mathcal{D}^{o p} \otimes \mathcal{D}\right), \phi:=\left(\phi^{\prime}, \phi^{\prime \prime}\right) \in \operatorname{Hom}_{\mathcal{D}^{o p} \otimes \mathcal{D}}\left((Y, X),\left(Y^{\prime}, X^{\prime}\right)\right)$, $f \in \operatorname{Hom}_{\mathcal{D}}(Y, X)$ and $c \in C$. We consider a collection

$$
\theta:=\left\{\theta_{X}: C \otimes C \longrightarrow \operatorname{End}_{\mathcal{D}}(X)\right\}_{X \in \mathrm{Ob}(\mathcal{D})}
$$

of $K$-linear maps satisfying the following conditions:

$$
\begin{gathered}
\left(\theta_{X}(c \otimes d)\right) \circ f=f_{\psi_{\psi}} \circ \theta_{Y}\left(c^{\psi} \otimes d^{\psi}\right), \\
\theta_{X}\left(c \otimes d_{1}\right) \otimes d_{2}=\left(\theta_{X}\left(c_{2} \otimes d\right)\right)_{\psi} \otimes c_{1}{ }^{\psi},
\end{gathered}
$$

for any $f \in \operatorname{Hom}_{\mathcal{D}}(Y, X)$. Let $V_{1}$ be the $K$-space consisting of all such $\theta$. Our first result gives conditions for the functors $\mathscr{F}$ and $\mathscr{G}$ to be separable.

Theorem A (see 3.7, 3.8, 3.10 and 3.11): Let $\mathcal{D}$ be a small $K$-linear category, $\left(C, \Delta_{C}, \varepsilon_{C}\right)$ be a $K$-coalgebra and let $(\mathcal{D}, C, \psi)$ be a right-right entwining structure.

(a) Let $V=\operatorname{Nat}\left(\mathscr{G} \mathscr{F}, 1_{\mathscr{M}(\psi)_{\mathcal{D}}^{C}}\right)$ be the space of natural transformations

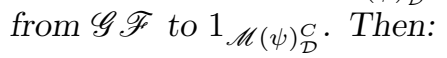

(1) There is an isomorphism $V \cong V_{1}$ of $K$-vector spaces.

(2) The functor $\mathscr{F}$ is separable if and only if there exists $\theta \in V_{1}$ such that

$$
\theta_{X} \circ \Delta_{C}=\varepsilon_{C} \cdot \operatorname{id}_{X} \quad \forall X \in \mathrm{Ob}(\mathcal{D}) .
$$

(b) Let $W=\operatorname{Nat}\left(1_{\mathrm{Mod}-\mathcal{D}}, \mathscr{F} \mathscr{G}\right)$ be the space of natural transformations from $1_{\text {Mod-D }}$ to $\mathscr{F} \mathscr{G}$. Then:

(1) There is an isomorphism of $K$-vector spaces from $W$ to

$$
W_{1}=\operatorname{Nat}(h, h \otimes C) .
$$

(2) The functor $\mathscr{G}$ is separable if and only if there exists $\eta \in W_{1}$ such that

$$
\left(\operatorname{id}_{h} \otimes \varepsilon_{C}\right) \eta=\mathrm{id}_{h} .
$$


The next result gives conditions for $(\mathscr{F}, \mathscr{G})$ to be a Frobenius pair.

Theorem B (see 3.14): Let $\mathcal{D}$ be a small $K$-linear category, $\left(C, \Delta_{C}, \varepsilon_{C}\right)$ be a $K$-coalgebra and let $(\mathcal{D}, C, \psi)$ be a right-right entwining structure. Then, $(\mathscr{F}, \mathscr{G})$ is a Frobenius pair if and only if there exist $\theta \in V_{1}$ and $\eta \in W_{1}$ such that the following conditions hold:

$$
\varepsilon_{C}(d) f=\sum \hat{f} \circ \theta_{X}\left(c_{f} \otimes d\right), \quad \varepsilon_{C}(d) f=\sum \hat{f}_{\psi} \circ \theta_{X}\left(d^{\psi} \otimes c_{f}\right),
$$

for any $f \in \operatorname{Hom}_{\mathcal{D}}(X, Y), d \in C$ and $\eta(X, Y)(f)=\sum \hat{f} \otimes c_{f}$.

More generally, the $\mathcal{D}$ - $\mathcal{D}$-bimodule $h \otimes C$ may be treated as a functor

$$
h \otimes C: \mathcal{D} \longrightarrow \mathscr{M}(\psi)_{\mathcal{D}}^{C}
$$

by setting (see Lemma 2.4)

$$
(h \otimes C)(Y):=\operatorname{Hom}_{\mathcal{D}}(-, Y) \otimes C, \quad(h \otimes C)(f)(Z)(g \otimes c):=f g \otimes c,
$$

for $f \in \operatorname{Hom}_{\mathcal{D}}(Y, X)$ and $g \otimes c \in \operatorname{Hom}_{\mathcal{D}}(Z, Y) \otimes C$. Additionally, let $C$ be a finite-dimensional coalgebra and let $C^{*}=\operatorname{Hom}(C, K)$ be the linear dual of $C$. Then, we show that there is a functor $C^{*} \otimes h: \mathcal{D} \longrightarrow \mathscr{M}(\psi)_{\mathcal{D}}^{C}$.

Theorem $\mathrm{C}$ (see 3.19): Let $(\mathcal{D}, C, \psi)$ be an entwining structure and let $C$ be a finite-dimensional coalgebra. Then, the following statements are equivalent:

(i) $(\mathscr{F}, \mathscr{G})$ is a Frobenius pair.

(ii) $C^{*} \otimes h$ and $h \otimes C$ are isomorphic as functors from $\mathcal{D}$ to $\mathscr{M}(\psi)_{\mathcal{D}}^{C}$.

In the final part of this paper, we study coalgebra Galois extensions of categories in a manner analogous to Brzeziński [3], Brzeziński and Hajac [6] and Caenepeel [11]. For this, we suppose that every morphism space $\operatorname{Hom}_{\mathcal{D}}(X, Y)$ carries the structure of a $C$-comodule

$$
\rho_{X Y}: \operatorname{Hom}_{\mathcal{D}}(X, Y) \longrightarrow \operatorname{Hom}_{\mathcal{D}}(X, Y) \otimes C, \quad f \mapsto \sum f_{0} \otimes f_{1} .
$$

This allows us to define a category $\mathcal{E}$ of $C$-coinvariants of $\mathcal{D}$ (see Definition 4.5). Further, we say that $\mathcal{D}$ is a $C$-Galois extension of $\mathcal{E}$ if the canonical map

$$
\operatorname{can}_{X}: h \otimes_{\mathcal{E}} \operatorname{Hom}_{\mathcal{D}}(X,-) \longrightarrow \operatorname{Hom}_{\mathcal{D}}(X,-) \otimes C
$$

is an isomorphism for each $X \in \mathrm{Ob}(\mathcal{D})$ (see Definition 4.7). We show that a $C$-Galois extension leads to a canonical entwining structure. 
Theorem D (see 4.9): Let $\mathcal{D}$ be a $C$-Galois extension of $\mathcal{E}$. Then, there exists a unique right-right entwining structure $(\mathcal{D}, C, \psi)$ which makes $\operatorname{Hom}_{\mathcal{D}}(-, Y)$ an object in $\mathscr{M}(\psi)_{\mathcal{D}}^{C}$ for every $Y \in \operatorname{Ob}(\mathcal{D})$ with its canonical $\mathcal{D}$-module structure and right $C$-coactions $\left\{\rho_{X Y}\right\}_{X \in \mathrm{Ob}(\mathcal{D})}$.

Conversely, under suitable conditions, an entwining structure $(\mathcal{D}, C, \psi)$ may be used to express $\mathcal{D}$ as a $C$-Galois extension. In that case, the category $\mathscr{M}(\psi)_{\mathcal{D}}^{C}$ reduces to the category of modules over the $C$-coinvariants of $\mathcal{D}$.

Theorem E (see 4.12 and 4.21): Let $C$ be a $K$-coalgebra and $\mathcal{D}$ be a small $K$ linear category such that $\operatorname{Hom}_{\mathcal{D}}(X, Y)$ has a right $C$-comodule structure $\rho_{X Y}$ for every $X, Y \in \mathrm{Ob}(\mathcal{D})$. Let $\mathcal{E}$ be the subcategory of $C$-coinvariants of $\mathcal{D}$. If there exists a convolution invertible collection

$$
\Phi=\left\{\Phi_{X Y}: C \longrightarrow \operatorname{Hom}_{\mathcal{D}}(X, Y)\right\}_{X, Y \in \mathrm{Ob}(\mathcal{D})}
$$

of right $C$-comodule maps, then the following are equivalent:

(i) $\mathcal{D}$ is a $C$-Galois extension of $\mathcal{E}$.

(ii) There exists a right-right entwining structure $(\mathcal{D}, C, \psi)$ such that $\operatorname{Hom}_{\mathcal{D}}(-, Y)$ is an object in $\mathscr{M}(\psi)_{\mathcal{D}}^{C}$ for every $Y \in \operatorname{Ob}(\mathcal{D})$ with its canonical $\mathcal{D}$-module structure and right $C$-coactions $\left\{\rho_{X Y}\right\}_{X \in \mathrm{Ob}(\mathcal{D})}$.

(iii) For any $f \in \operatorname{Hom}_{\mathcal{D}}(X, Y)$, the morphism $\sum f_{0} \circ \Phi_{Z X}^{\prime}\left(f_{1}\right) \in \operatorname{Hom}_{\mathcal{E}}(Z, Y)$ for every $Z \in \mathrm{Ob}(\mathcal{D})$, where $\Phi^{\prime}$ is the convolution inverse of $\Phi$.

In this case, the categories $\mathscr{M}(\psi)_{\mathcal{D}}^{C}$ and Mod-E $\mathcal{E}$ are equivalent.

Notations. Throughout the paper, $K$ is a field, $C$ is a $K$-coalgebra with comultiplication $\Delta_{C}$ and counit $\varepsilon_{C}$. We shall use Sweedler's notation for the coproduct $\Delta_{C}(c)=c_{1} \otimes c_{2}$, and for a coaction

$$
\rho_{M}: M \longrightarrow M \otimes C, \quad \rho_{M}(m)=m_{0} \otimes m_{1}
$$

with the summation omitted. We denote by $C^{*}$ the linear dual of $C$. Sometimes when the coaction is clear from context, we will omit the subscript.

\section{Entwining structures}

In this section, we introduce a categorical generalization of entwining structures and entwined modules. We prove that the category of entwined modules is a Grothendieck category. We begin by recalling the definition of modules over a category (see, for instance, $[29,23])$. 
Definition 2.1: A right module over a small $K$-linear category $\mathcal{D}$ is a $K$-linear functor $\mathcal{D}^{o p} \longrightarrow$ Vect $_{K}$, where Vect $_{K}$ denotes the category of $K$-vector spaces. Similarly, a left module over $\mathcal{D}$ is a $K$-linear functor $\mathcal{D} \longrightarrow \operatorname{Vect}_{K}$. The category of all right (resp. left) modules over $\mathcal{D}$ will be denoted by $\operatorname{Mod}-\mathcal{D}$ (resp. $\mathcal{D}$-Mod).

For each $X \in \operatorname{Ob}(\mathcal{D})$, the representable functors

$$
\mathbf{h}_{X}:=\operatorname{Hom}_{\mathcal{D}}(-, X) \quad \text { and } \quad X \mathbf{h}:=\operatorname{Hom}_{\mathcal{D}}(X,-)
$$

are examples of right and left modules over $\mathcal{D}$ respectively. Unless otherwise mentioned, by a $\mathcal{D}$-module we will always mean a right $\mathcal{D}$-module.

Let $C$ be a $K$-coalgebra and let $\mathcal{D}$ be a small $K$-linear category. Suppose that we have a collection of $K$-linear maps

$$
\psi=\left\{\psi_{X Y}: C \otimes \operatorname{Hom}_{\mathcal{D}}(X, Y) \longrightarrow \operatorname{Hom}_{\mathcal{D}}(X, Y) \otimes C\right\}_{(X, Y) \in \operatorname{Ob}(\mathcal{D})^{2} .}
$$

We use the notation $\psi_{X Y}(c \otimes f)=f_{\psi} \otimes c^{\psi}$ for $c \in C$ and $f \in \operatorname{Hom}_{\mathcal{D}}(X, Y)$. We will say that the tuple $(\mathcal{D}, C, \psi)$ is a (right-right) entwining structure if the following conditions hold:

$$
\begin{aligned}
(g f)_{\psi} \otimes c^{\psi} & =g_{\psi} f_{\psi} \otimes c^{\psi \psi}, \\
\varepsilon_{C}\left(c^{\psi}\right)\left(f_{\psi}\right) & =\varepsilon_{C}(c) f, \\
f_{\psi} \otimes \Delta_{C}\left(c^{\psi}\right) & =f_{\psi_{\psi}} \otimes c_{1}{ }^{\psi} \otimes c_{2}{ }^{\psi}, \\
\psi_{X X}\left(c \otimes \mathrm{id}_{X}\right) & =\operatorname{id}_{X} \otimes c,
\end{aligned}
$$

for each $f \in \operatorname{Hom}_{\mathcal{D}}(X, Y), g \in \operatorname{Hom}_{\mathcal{D}}(Y, Z)$ and $c \in C$. Throughout this paper, $(\mathcal{D}, C, \psi)$ will always be an entwining structure. A morphism between entwining structures $\left(\mathcal{D}^{\prime}, C^{\prime}, \psi^{\prime}\right)$ and $(\mathcal{D}, C, \psi)$ is a pair $(\mathscr{F}, \sigma)$ where $\mathscr{F}: \mathcal{D}^{\prime} \longrightarrow \mathcal{D}$ is a functor and $\sigma: C^{\prime} \longrightarrow C$ is a counital coalgebra map such that

$$
\mathscr{F}\left(f^{\prime}{ }_{\psi^{\prime}}\right) \otimes \sigma\left(c^{\prime \psi^{\prime}}\right)=\mathscr{F}\left(f^{\prime}\right)_{\psi} \otimes \sigma\left(c^{\prime}\right)^{\psi}
$$

for any $c^{\prime} \otimes f^{\prime} \in C^{\prime} \otimes \operatorname{Hom}_{\mathcal{D}^{\prime}}\left(X^{\prime}, Y^{\prime}\right)$ where $X^{\prime}, Y^{\prime} \in \operatorname{Ob}\left(\mathcal{D}^{\prime}\right)$.

Definition 2.2: Let $\mathcal{M}$ be a right $\mathcal{D}$-module with a given right $C$-comodule structure $\rho_{\mathcal{M}(Y)}: \mathcal{M}(Y) \longrightarrow \mathcal{M}(Y) \otimes C$ on $\mathcal{M}(Y)$ for each $Y \in \mathrm{Ob}(\mathcal{D})$. Then, $\mathcal{M}$ is said to be an entwined module over $(\mathcal{D}, C, \psi)$ if the following compatibility condition holds:

$$
\rho_{\mathcal{M}(Y)}(\mathcal{M}(f)(m))=(\mathcal{M}(f)(m))_{0} \otimes(\mathcal{M}(f)(m))_{1}=\mathcal{M}\left(f_{\psi}\right)\left(m_{0}\right) \otimes m_{1}{ }^{\psi}
$$


for every $f \in \operatorname{Hom}_{\mathcal{D}}(Y, X)$ and $m \in \mathcal{M}(X)$. We denote by $\mathscr{M}(\psi)_{\mathcal{D}}^{C}$ the category whose objects are entwined modules over $(\mathcal{D}, C, \psi)$ and whose morphisms are given by

$$
\begin{array}{r}
\operatorname{Hom}_{\mathscr{M}(\psi)_{\mathcal{D}}^{C}}(\mathcal{M}, \mathcal{N}):=\left\{\eta \in \operatorname{Hom}_{\mathrm{Mod}-\mathcal{D}}(\mathcal{M}, \mathcal{N}) \mid \eta(X): \mathcal{M}(X) \longrightarrow \mathcal{N}(X)\right. \\
\text { is } C \text {-colinear } \forall X \in \mathrm{Ob}(\mathcal{D})\}
\end{array}
$$

We now give an important example of entwining structures.

Example 2.3: Let $\mathcal{D}$ be a right co- $H$-category (see [16] or the description in [2, Definition 2.4]) and $C$ be a right $H$-module coalgebra. Then, the triple $(\mathcal{D}, C, \psi)$ is an entwining structure, where $\psi$ is given by

$$
\begin{aligned}
\psi_{X Y}: C \otimes \operatorname{Hom}_{\mathcal{D}}(X, Y) \longrightarrow C \otimes \operatorname{Hom}_{\mathcal{D}}(X, Y) \otimes H \stackrel{\cong}{\longrightarrow} \operatorname{Hom}_{\mathcal{D}}(X, Y) \otimes C \otimes H \\
\longrightarrow \operatorname{Hom}_{\mathcal{D}}(X, Y) \otimes C .
\end{aligned}
$$

Explicitly, we have

$$
\psi_{X Y}(c \otimes f):=f_{0} \otimes c f_{1}
$$

for any $f \in \operatorname{Hom}_{\mathcal{D}}(X, Y)$ and $c \in C$. In this case, an entwined module is precisely a right $\mathcal{D}$-module with a given right $C$-comodule structure on $\mathcal{M}(X)$ for each $X \in \mathrm{Ob}(\mathcal{D})$ and satisfying the following compatibility condition:

$$
(\mathcal{M}(f)(m))_{0} \otimes(\mathcal{M}(f)(m))_{1}=\mathcal{M}\left(f_{0}\right)\left(m_{0}\right) \otimes m_{1} f_{1}
$$

We will refer to these modules as (right-right) Doi-Hopf modules and their category will be denoted by $\mathscr{M}_{\mathcal{D}}^{C}$. If $\mathcal{D}$ is a right co- $H$-category with a single object, i.e., an $H$-comodule algebra, then $\mathscr{M}_{\mathcal{D}}^{C}$ recovers the classical notion of Doi-Hopf modules (see [18]). In the particular case where $C=H$, the right-right Doi-Hopf modules have been referred to as relative Hopf modules in $[2, \S 5]$.

Lemma 2.4: Let $(\mathcal{D}, C, \psi)$ be an entwining structure and let $\mathcal{M}$ be a right $\mathcal{D}$-module. Then, we may obtain an object $\mathcal{M} \otimes C \in \mathscr{M}(\psi)_{\mathcal{D}}^{C}$ by setting

$$
\begin{aligned}
(\mathcal{M} \otimes C)(X) & :=\mathcal{M}(X) \otimes C \\
(\mathcal{M} \otimes C)(f)(m \otimes c) & :=\mathcal{M}\left(f_{\psi}\right)(m) \otimes c^{\psi}
\end{aligned}
$$

for $X \in O b(\mathcal{D}), \quad f \in \operatorname{Hom}_{\mathcal{D}}(Y, X)$ and $m \otimes c \in \mathcal{M}(X) \otimes C$. In fact, this determines a functor from $\operatorname{Mod}-\mathcal{D}$ to $\mathscr{M}(\psi)_{\mathcal{D}}^{C}$. 
Proof. The fact that $\mathcal{M} \otimes C$ is a right $\mathcal{D}$-module follows from (2.1). For each $X \in \operatorname{Ob}(\mathcal{D})$, it may be verified that $\mathcal{M}(X) \otimes C$ has a right $C$-comodule structure given by

$$
\pi_{\mathcal{M}(X) \otimes C}^{r}(m \otimes c):=\left(\operatorname{id}_{\mathcal{M}(X)} \otimes \Delta_{C}\right)(m \otimes c)=m \otimes c_{1} \otimes c_{2} .
$$

It remains to check the compatibility condition in (2.5). By definition, we have

$$
\begin{aligned}
\left(\mathcal{M}\left(f_{\psi}\right)(m) \otimes c^{\psi}\right)_{0} \otimes & \left(\mathcal{M}\left(f_{\psi}\right)(m) \otimes c^{\psi}\right)_{1} \\
& =\mathcal{M}\left(f_{\psi}\right)(m) \otimes\left(c^{\psi}\right)_{1} \otimes\left(c^{\psi}\right)_{2} \\
& =\mathcal{M}\left(f_{\psi_{\psi}}\right)(m) \otimes c_{1}{ }^{\psi} \otimes c_{2}{ }^{\psi} \\
& =(\mathcal{M} \otimes C)\left(f_{\psi}\right)\left(m \otimes c_{1}\right) \otimes c_{2}{ }^{\psi} .
\end{aligned}
$$

Lemma 2.5: Let $(\mathcal{D}, C, \psi)$ be an entwining structure and $N$ be a right $C$-comodule. Then, for each $X \in \mathrm{Ob}(\mathcal{D})$ we may obtain an object $N \otimes \mathbf{h}_{X} \in \mathscr{M}(\psi)_{\mathcal{D}}^{C}$ by setting

$$
\begin{aligned}
\left(N \otimes \mathbf{h}_{X}\right)(Y) & :=N \otimes \mathbf{h}_{X}(Y), \\
\left(N \otimes \mathbf{h}_{X}\right)(f)(n \otimes g) & :=n \otimes g f,
\end{aligned}
$$

for $Y \in \operatorname{Ob}(\mathcal{D}), f \in \operatorname{Hom}_{\mathcal{D}}(Z, Y), n \otimes g \in N \otimes \mathbf{h}_{X}(Y)$. In fact, this determines a functor from Comod $-C$ to $\mathscr{M}(\psi)_{\mathcal{D}}^{C}$.

Proof. By definition, it follows that $N \otimes \mathbf{h}_{X}$ is a right $\mathcal{D}$-module. Further, for each $Y \in \mathrm{Ob}(\mathcal{D})$, we define a $K$-linear map

$$
\sigma_{N \otimes \mathbf{h}_{X}(Y)}^{r}: N \otimes \mathbf{h}_{X}(Y) \longrightarrow N \otimes \mathbf{h}_{X}(Y) \otimes C
$$

as follows:

$$
\sigma_{N \otimes \mathbf{h}_{X}(Y)}^{r}(n \otimes g):=n_{0} \otimes g_{\psi} \otimes n_{1}{ }^{\psi} .
$$

We now verify that the map defined in (2.9) makes $N \otimes \mathbf{h}_{X}(Y)$ a right $C$-comodule. We have

$$
\begin{aligned}
\left(\sigma^{r} \otimes \operatorname{id}_{C}\right) \sigma^{r}(n \otimes g) & =\left(\sigma^{r} \otimes \operatorname{id}_{C}\right)\left(n_{0} \otimes g_{\psi} \otimes n_{1}{ }^{\psi}\right) \\
& =n_{0} \otimes g_{\psi} \otimes n_{1}{ } \otimes n_{2}{ }^{\psi} \\
& =n_{0} \otimes g_{\psi} \otimes \Delta_{C}\left(n_{1}{ }^{\psi}\right) \\
& =\left(\operatorname{id}_{N \otimes \mathbf{h}_{X}(Y)} \otimes \Delta_{C}\right) \sigma^{r}(n \otimes g) .
\end{aligned}
$$


Moreover, using (2.2) we have

$$
\begin{aligned}
\left(\operatorname{id}_{N \otimes \mathbf{h}_{X}(Y)} \otimes \varepsilon_{C}\right) \sigma^{r}(n \otimes g) & =\left(\operatorname{id}_{N \otimes \mathbf{h}_{X}(Z)} \otimes \varepsilon_{C}\right)\left(n_{0} \otimes g_{\psi} \otimes n_{1}{ }^{\psi}\right) \\
& =n_{0} \otimes \varepsilon_{C}\left(n_{1} \psi\right) g_{\psi}=n_{0} \otimes \varepsilon_{C}\left(n_{1}\right) g=n \otimes g .
\end{aligned}
$$

It remains to verify the condition in (2.5). We have

$$
\begin{aligned}
\left(\left(N \otimes \mathbf{h}_{X}\right)(f)(n \otimes g)\right)_{0} \otimes & \left(\left(N \otimes \mathbf{h}_{X}\right)(f)(n \otimes g)\right)_{1} \\
& =n_{0} \otimes(g f)_{\psi} \otimes n_{1}{ }^{\psi} \\
& =n_{0} \otimes g_{\psi} f_{\psi} \otimes n_{1} \psi^{\psi} \\
& =\left(N \otimes \mathbf{h}_{X}\right)\left(f_{\psi}\right)\left(n_{0} \otimes g_{\psi}\right) \otimes n_{1}{ }^{\psi}{ }^{\psi} \\
& =\left(N \otimes \mathbf{h}_{X}\right)\left(f_{\psi}\right)\left((n \otimes g)_{0}\right) \otimes(n \otimes g)_{1}{ }^{\psi}
\end{aligned}
$$

It follows from Lemma 2.4 and Lemma 2.5 that both $\mathbf{h}_{Y} \otimes C$ and $C \otimes \mathbf{h}_{Y}$ are objects in $\mathscr{M}(\psi)_{\mathcal{D}}^{C}$ for every $Y \in \operatorname{Ob}(\mathcal{D})$.

Lemma 2.6: Let $(\mathcal{D}, C, \psi)$ be an entwining structure. Then, for each $Y \in \operatorname{Ob}(\mathcal{D})$, we get a morphism $\Psi_{Y}: C \otimes \mathbf{h}_{Y} \longrightarrow \mathbf{h}_{Y} \otimes C$ in $\mathscr{M}(\psi)_{\mathcal{D}}^{C}$ given by $\Psi_{Y}(X):=\psi_{X Y}$.

Proof. First we verify that $\Psi_{Y}$ is a morphism of right $\mathcal{D}$-modules. For any $f \in \operatorname{Hom}_{\mathcal{D}}\left(X^{\prime}, X\right), g \in \operatorname{Hom}_{\mathcal{D}}(X, Y)$ and $c \in C$, we have

$$
\begin{aligned}
\left(\left(\mathbf{h}_{Y} \otimes C\right)(f)\right) \psi_{X Y}(c \otimes g) & =\left(\mathbf{h}_{Y} \otimes C\right)(f)\left(g_{\psi} \otimes c^{\psi}\right)=g_{\psi} f_{\psi} \otimes c^{\psi^{\psi}}=(g f)_{\psi} \otimes c^{\psi} \\
& =\psi_{X^{\prime} Y}(c \otimes g f)=\psi_{X^{\prime} Y}\left(C \otimes \mathbf{h}_{Y}\right)(f)(c \otimes g) .
\end{aligned}
$$

Next, we will show that $\Psi_{Y}(X)$ is $C$-colinear for every $X \in \mathrm{Ob}(\mathcal{D})$. We have

$$
\begin{aligned}
\left(\psi_{X Y}(c \otimes g)\right)_{0} \otimes & \left(\psi_{X Y}(c \otimes g)\right)_{1} \\
& =g_{\psi} \otimes \Delta_{C}\left(c^{\psi}\right) \\
& =g_{\psi} \otimes c_{1} \psi \otimes c_{2}{ }^{\psi} \\
& =\left(\psi_{X Y} \otimes \mathrm{id}\right)(c \otimes g)_{0} \otimes(c \otimes g)_{1}
\end{aligned}
$$

We now recall from $[22, \S 3]$ and $[23]$ the notion of a finitely generated module over a category. Given $\mathcal{M} \in \operatorname{Mod}-\mathcal{D}$, we set

$$
\mathrm{el}(\mathcal{M}):=\coprod_{X \in \mathrm{Ob}(\mathcal{D})} \mathcal{M}(X)
$$

to be the collection of all elements of $\mathcal{M}$. Since $\mathcal{D}$ is small, we note that el $(\mathcal{M})$ is a set. If $m \in \operatorname{el}(\mathcal{M})$ is such that $m \in \mathcal{M}(X)$, we will write $|m|=X$. 
Definition 2.7: Let $\mathcal{D}$ be a small preadditive category and let $\mathcal{M}$ be a right $\mathcal{D}$-module. For each $m \in \operatorname{el}(\mathcal{M})$, we consider the corresponding morphism $\eta_{m}: \mathbf{h}_{|m|} \longrightarrow \mathcal{M}$. A family of elements $\left\{m_{i} \in \operatorname{el}(\mathcal{M})\right\}_{i \in I}$ is said to be a generating set for $\mathcal{M}$ if the induced morphism

$$
\eta: \bigoplus_{i \in I} \mathbf{h}_{\left|m_{i}\right|} \longrightarrow \mathcal{M}, \quad\left(0, \ldots, 0, \operatorname{id}_{\left|m_{i}\right|}, 0, \ldots, 0\right) \mapsto m_{i}
$$

is an epimorphism in Mod-D. In other words, every element $m \in \operatorname{el}(\mathcal{M})$ may be expressed as a sum $m=\sum_{i \in I} \mathcal{M}\left(f_{i}\right)\left(m_{i}\right)$, where each $f_{i} \in \operatorname{Hom}_{\mathcal{D}}\left(|m|,\left|m_{i}\right|\right)$ and all but finitely many $\left\{f_{i}\right\}_{i \in I}$ are zero.

Lemma 2.8: Let $(\mathcal{D}, C, \psi)$ be an entwining structure and let $\mathcal{M}$ be an entwined module. We consider an element $m \in \operatorname{el}(\mathcal{M})$. Then, there exists a finite-dimensional $C$-subcomodule $V_{m}$ of $\mathcal{M}(|m|)$ containing $m$ and a morphism $\eta_{m}: V_{m} \otimes \mathbf{h}_{|m|} \longrightarrow \mathcal{M}$ in $\mathscr{M}(\psi)_{\mathcal{D}}^{C}$ such that $\eta_{m}(|m|)\left(m \otimes \operatorname{id}_{|m|}\right)=m$.

Proof. By [17, Theorem 2.1.7], we know that there exists a finite-dimensional $C$ subcomodule $V_{m} \subseteq \mathcal{M}(|m|)$ such that $m \in V_{m}$. Now, we consider the $\mathcal{D}$-module morphism $\eta_{m}: V_{m} \otimes \mathbf{h}_{|m|} \longrightarrow \mathcal{M}$ defined by setting $\eta_{m}(Y)(v \otimes f):=\mathcal{M}(f)(v)$ for any $Y \in \operatorname{Ob}(\mathcal{D}), f \in \operatorname{Hom}_{\mathcal{D}}(Y,|m|)$ and $v \in V_{m}$. We also have

$$
\begin{aligned}
\rho_{\mathcal{M}(Y)}\left(\eta_{m}(Y)(v \otimes f)\right) & =\rho_{\mathcal{M}(Y)}(\mathcal{M}(f)(v)) \\
& =\mathcal{M}\left(f_{\psi}\right)\left(v_{0}\right) \otimes v_{1}^{\psi}=\eta_{m}(Y)\left(v_{0} \otimes f_{\psi}\right) \otimes v_{1}^{\psi} \\
& =\left(\eta_{m}(Y) \otimes \operatorname{id}_{C}\right)\left(\rho_{V_{m} \otimes \mathbf{h}_{|m|}(Y)}(v \otimes f)\right)
\end{aligned}
$$

This shows that $\eta_{m}(Y)$ is $C$-colinear for each $Y \in \operatorname{Ob}(\mathcal{D})$. Hence, $\eta_{m}$ is a morphism in $\mathscr{M}(\psi)_{\mathcal{D}}^{C}$ such that $\eta_{m}(|m|)\left(m \otimes \operatorname{id}_{|m|}\right)=m$.

Proposition 2.9: Let $(\mathcal{D}, C, \psi)$ be an entwining structure. Then, the category $\mathscr{M}(\psi)_{\mathcal{D}}^{C}$ of entwined modules is a Grothendieck category.

Proof. Given a morphism $\eta: \mathcal{M} \longrightarrow \mathcal{N}$ in $\mathscr{M}(\psi)_{\mathcal{D}}^{C}$, let $\operatorname{Ker}(\eta)$ and $\operatorname{Coker}(\eta)$ be respectively the kernel and cokernel in $\operatorname{Mod}-\mathcal{D}$. Since Comod-C is an abelian category, we know that $\operatorname{Ker}(\eta)(X), \operatorname{Coker}(\eta)(X) \in \operatorname{Comod}-C$ for each $X \in \mathrm{Ob}(\mathcal{D})$. It is easily seen that $\operatorname{Ker}(\eta)$ and $\operatorname{Coker}(\eta)$ satisfy the compatibility condition in $(2.5)$, i.e., $\operatorname{Ker}(\eta)$, $\operatorname{Coker}(\eta) \in \mathscr{M}(\psi)_{\mathcal{D}}^{C}$. Since limits and colimits in $\mathscr{M}(\psi)_{\mathcal{D}}^{C}$ are obtained from those in Mod-D and Comod-C, it is clear that $\mathscr{M}(\psi)_{\mathcal{D}}^{C}$ is a cocomplete abelian category satisfying (AB5). 
By Lemma 2.8, there is an epimorphism

$$
\bigoplus_{m \in \operatorname{el}(\mathcal{M})} \eta_{m}: \bigoplus_{m \in \operatorname{el}(\mathcal{M})} V_{m} \otimes \mathbf{h}_{|m|} \longrightarrow \mathcal{M}
$$

for any $\mathcal{M} \in \mathscr{M}(\psi)_{\mathcal{D}}^{C}$. As such, the collection $\left\{V \otimes \mathbf{h}_{X}\right\}$, where $X$ ranges over all objects in $\mathcal{D}$ and $V$ ranges over all (isomorphism classes of) finitedimensional $C$-comodules gives a set of generators for $\mathscr{M}(\psi)_{\mathcal{D}}^{C}$ in the sense of [20, Proposition 1.9.1].

Corollary 2.10: The category $\mathscr{M}_{\mathcal{D}}^{C}$ of Doi-Hopf modules is a Grothendieck category.

\section{Separability and Frobenius conditions}

Let $\mathscr{F}: \mathscr{M}(\psi)_{\mathcal{D}}^{C} \longrightarrow$ Mod-D be the forgetful functor. The next result shows that the functor $\mathscr{F}$ has a right adjoint.

Lemma 3.1: The forgetful functor $\mathscr{F}: \mathscr{M}(\psi)_{\mathcal{D}}^{C} \longrightarrow$ Mod-D has a right adjoint $\mathscr{G}: \operatorname{Mod}-\mathcal{D} \longrightarrow \mathscr{M}(\psi)_{\mathcal{D}}^{C}$ given by

$$
\mathscr{G}(\mathcal{N}):=\mathcal{N} \otimes C
$$

for each $\mathcal{N} \in \operatorname{Mod}-\mathcal{D}$.

Proof. From Lemma 2.4, we know that $\mathscr{G}(\mathcal{N})=\mathcal{N} \otimes C \in \mathscr{M}(\psi)_{\mathcal{D}}^{C}$ for each $\mathcal{N} \in \operatorname{Mod}-\mathcal{D}$. We define $\alpha: \operatorname{Hom}_{\mathscr{M}(\psi)_{\mathcal{D}}^{C}}(\mathcal{M}, \mathscr{G}(\mathcal{N})) \longrightarrow \operatorname{Hom}_{\text {Mod-D }}(\mathscr{F}(\mathcal{M}), \mathcal{N})$ by setting

$$
\alpha(\xi)(X)(m):=\left(\operatorname{id}_{\mathcal{N}(X)} \otimes \varepsilon_{C}\right)(\xi(X)(m))
$$

for each $\xi: \mathcal{M} \longrightarrow \mathcal{N} \otimes C$ in $\mathscr{M}(\psi)_{\mathcal{D}}^{C}, X \in \mathrm{Ob}(\mathcal{D})$ and $m \in \mathcal{M}(X)$.

We also define $\beta: \operatorname{Hom}_{\text {Mod -D }}(\mathscr{F}(\mathcal{M}), \mathcal{N}) \longrightarrow \operatorname{Hom}_{\mathscr{M}(\psi)_{\mathcal{D}}^{C}}(\mathcal{M}, \mathscr{G}(\mathcal{N}))$ by setting

$$
\beta(\eta)(X)(m):=\eta(X)\left(m_{0}\right) \otimes m_{1}
$$

for each $\eta: \mathcal{M} \longrightarrow \mathcal{N}$ in $\operatorname{Mod}-\mathcal{D}, X \in \mathrm{Ob}(\mathcal{D})$ and $m \in \mathcal{M}(X)$. First we check that $\alpha(\xi)$ and $\beta(\eta)$ are morphisms in $\operatorname{Mod}-\mathcal{D}$ and $\mathscr{M}(\psi)_{\mathcal{D}}^{C}$ respectively. Using the fact that $\operatorname{id}_{\mathcal{N}} \otimes \varepsilon_{C}: \mathcal{N} \otimes C \longrightarrow \mathcal{N}$ and $\xi$ are right $\mathcal{D}$-module morphisms, for 
any $f \in \operatorname{Hom}_{\mathcal{D}}(Y, X)$, we have

$$
\begin{aligned}
\mathcal{N}(f)(\alpha(\xi)(X)(m)) & =\mathcal{N}(f)\left(\left(\operatorname{id}_{\mathcal{N}(X)} \otimes \varepsilon_{C}\right)(\xi(X)(m))\right) \\
& =\left(\operatorname{id}_{\mathcal{N}(Y)} \otimes \varepsilon_{C}\right)\left(\mathcal{N}(f) \otimes \operatorname{id}_{C}\right)(\xi(X)(m)) \\
& =\left(\operatorname{id}_{\mathcal{N}(Y)} \otimes \varepsilon_{C}\right)(\xi(Y) \mathcal{M}(f)(m)) \\
& =\alpha(\xi)(Y)(\mathcal{M}(f)(m)) .
\end{aligned}
$$

We also have

$$
\begin{aligned}
(\mathcal{N} \otimes C)(f)(\beta(\eta)(X)(m)) \\
=(\mathcal{N} \otimes C)(f)\left(\eta(X)\left(m_{0}\right) \otimes m_{1}\right) \\
=\mathcal{N}\left(f_{\psi}\right) \eta(X)\left(m_{0}\right) \otimes m_{1} \psi \\
=\eta(Y) \mathcal{M}\left(f_{\psi}\right)\left(m_{0}\right) \otimes m_{1} \psi \\
=\eta(Y)\left((\mathcal{M}(f)(m))_{0}\right) \otimes(\mathcal{M}(f)(m))_{1} \\
=\beta(\eta)(Y)(\mathcal{M}(f)(m))
\end{aligned}
$$

Moreover, it is easy to see that $\beta(\eta)(X)$ is $C$-colinear for each $X \in \mathrm{Ob}(\mathcal{D})$. We now verify that $\alpha$ and $\beta$ are inverses to each other:

$$
\begin{aligned}
& \beta(\alpha(\xi))(X)(m) \\
& =\alpha(\xi)(X)\left(m_{0}\right) \otimes m_{1} \\
& =\left(\operatorname{id}_{\mathcal{N}(X)} \otimes \varepsilon_{C}\right)\left(\xi(X)\left(m_{0}\right)\right) \otimes m_{1} \\
& =\left(\operatorname{id}_{\mathcal{N}(X)} \otimes \varepsilon_{C} \otimes \operatorname{id}_{C}\right)\left(\xi(X) \otimes \operatorname{id}_{C}\right) \rho_{\mathcal{M}(X)}(m) \\
& =\left(\operatorname{id}_{\mathcal{N}(X)} \otimes \varepsilon_{C} \otimes \operatorname{id}_{C}\right) \pi_{\mathcal{N}(X) \otimes C}^{r}(\xi(X)(m)) \quad(\xi(X) \text { is } C \text {-colinear }) \\
& =\xi(X)(m)
\end{aligned}
$$

Further, we have $\alpha(\beta(\eta))(X)(m)=\eta(X)\left(m_{0}\right) \varepsilon_{C}\left(m_{1}\right)=\eta(X)(m)$. This proves the result.

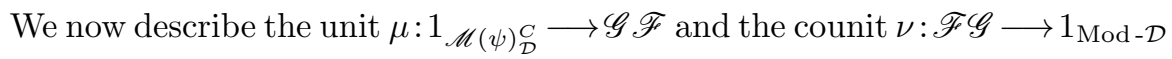
of the adjunction in Lemma 3.1:

$$
\begin{aligned}
\mu(\mathcal{M}): \mathcal{M} \longrightarrow \mathcal{M} \otimes C & \mu(\mathcal{M})(X)(m)=m_{0} \otimes m_{1}, \\
\nu(\mathcal{N})=\operatorname{id}_{\mathcal{N}} \otimes \varepsilon_{C}: \mathcal{N} \otimes C \longrightarrow \mathcal{N} & \nu(\mathcal{N})(X)(n \otimes c)=\varepsilon_{C}(c) n,
\end{aligned}
$$

for each $\mathcal{M} \in \mathscr{M}(\psi)_{\mathcal{D}}^{C}, \mathcal{N} \in \operatorname{Mod}-\mathcal{D}, X \in \operatorname{Ob}(\mathcal{D})$. 
We recall that a functor $F: \mathcal{A} \longrightarrow \mathcal{B}$ between arbitrary categories is said to be separable if the natural transformation

$$
\eta: \operatorname{Hom}_{\mathcal{A}}(-,-) \longrightarrow \operatorname{Hom}_{\mathcal{B}}(F(-), F(-))
$$

induced by $F$ is a split monomorphism (see [25], [26, §1]). The following result provides a characterization of separable functors.

Theorem $3.2([26$, Theorem 1.2]): Let $F: \mathcal{A} \longrightarrow \mathcal{B}$ be a functor which has a right adjoint $G: \mathcal{B} \longrightarrow \mathcal{A}$. Let $\mu$ and $\nu$ be the unit and counit of this adjunction respectively. Then:

(i) $F$ is separable if and only if there exists $v \in \operatorname{Nat}\left(G F, 1_{\mathcal{A}}\right)$ such that $v \circ \mu=1_{\mathcal{A}}$, the identity natural transformation on $\mathcal{A}$.

(ii) $G$ is separable if and only if there exists $\zeta \in \operatorname{Nat}\left(1_{\mathcal{B}}, F G\right)$ such that $\nu \circ \zeta=1_{\mathcal{B}}$, the identity natural transformation on $\mathcal{B}$.

3.1. Separability Conditions. Let $(\mathcal{D}, C, \psi)$ be an entwining structure. We now investigate the separability of the forgetful functor $\mathscr{F}: \mathscr{M}(\psi)_{\mathcal{D}}^{C} \longrightarrow$ Mod-D . Since $\mathscr{F}$ has a right adjoint $\mathscr{G}$, it follows from Theorem 3.2 that the functor $\mathscr{F}$ is separable if and only if there exists a natural transformation $v: \mathscr{G} \mathscr{F} \longrightarrow 1_{\mathscr{M}(\psi)_{\mathcal{D}}^{C}}$ such that $v \circ \mu=1_{\mathscr{M}(\psi)_{\mathcal{D}}^{C}}$, where $\mu$ is the unit of the adjunction as explained in (3.1). Throughout Section 3,

$$
V:=\operatorname{Nat}\left(\mathscr{G} \mathscr{F}, 1_{\mathscr{M}(\psi)_{\mathcal{D}}^{C}}\right)
$$

will denote the $K$-space of all natural transformations from $\mathscr{G} \mathscr{F}$ to $1_{\mathscr{M}(\psi)_{\mathcal{D}}^{C}}$. We will shortly give another useful interpretation of $V$. We start by proving a few preparatory results required for this.

We recall from Lemma 2.4 and Lemma 2.5 that both $\mathbf{h}_{Y} \otimes C$ and $C \otimes \mathbf{h}_{Y}$ are objects in $\mathscr{M}(\psi)_{\mathcal{D}}^{C}$ for every $Y \in \operatorname{Ob}(\mathcal{D})$. We define a functor $\mathbf{h} \otimes C: \mathcal{D} \longrightarrow \mathscr{M}(\psi)_{\mathcal{D}}^{C}$ as

$$
\begin{gathered}
(\mathbf{h} \otimes C)(Y):=\mathbf{h}_{Y} \otimes C, \\
(\mathbf{h} \otimes C)(f)(Z)(g \otimes c):=f g \otimes c,
\end{gathered}
$$

for $f \in \operatorname{Hom}_{\mathcal{D}}(Y, X), g \in \mathbf{h}_{Y}(Z)$ and $c \in C$. Similarly, we may also obtain a functor $\mathbf{h} \otimes C \otimes C: \mathcal{D} \longrightarrow \mathscr{M}(\psi)_{\mathcal{D}}^{C}$. 
Lemma 3.3: Let $f \in \operatorname{Hom}_{\mathcal{D}}(Y, X)$. For any $v \in V$ and $c, d \in C$, we have

$$
\begin{aligned}
\left(\left(\mathrm{id}_{\mathbf{h}_{X}} \otimes \varepsilon_{C}\right)\right. & \left.v\left(\mathbf{h}_{X} \otimes C\right)\right)(Y)(f \otimes c \otimes d) \\
= & f \circ\left(\left(\varepsilon_{C} \otimes \operatorname{id}_{\mathbf{h}_{Y}}\right) v\left(C \otimes \mathbf{h}_{Y}\right)\right)(Y)\left(c \otimes \mathrm{id}_{Y} \otimes d\right) .
\end{aligned}
$$

In particular, we have

$$
\begin{aligned}
\left(\left(\mathrm{id}_{\mathbf{h}_{X}} \otimes \varepsilon_{C}\right) v\left(\mathbf{h}_{X} \otimes C\right)\right)(X)\left(\mathrm{id}_{X} \otimes c \otimes d\right) & \\
= & \left(\left(\varepsilon_{C} \otimes \mathrm{id}_{\mathbf{h}_{X}}\right) v\left(C \otimes \mathbf{h}_{X}\right)\right)(X)\left(c \otimes \mathrm{id}_{X} \otimes d\right) .
\end{aligned}
$$

Proof. A morphism $f: Y \longrightarrow X$ in $\mathcal{D}$ induces morphisms $\mathbf{h}_{Y} \otimes C \longrightarrow \mathbf{h}_{X} \otimes C$ and $\mathbf{h}_{Y} \otimes C \otimes C \longrightarrow \mathbf{h}_{X} \otimes C \otimes C$ in $\mathscr{M}(\psi)_{\mathcal{D}}^{C}$ as explained in (3.4). Since $v: \mathscr{G} \mathscr{F} \longrightarrow 1_{\mathscr{M}(\psi)_{\mathcal{D}}^{C}}$ is a natural transformation, it follows that the following diagram commutes:

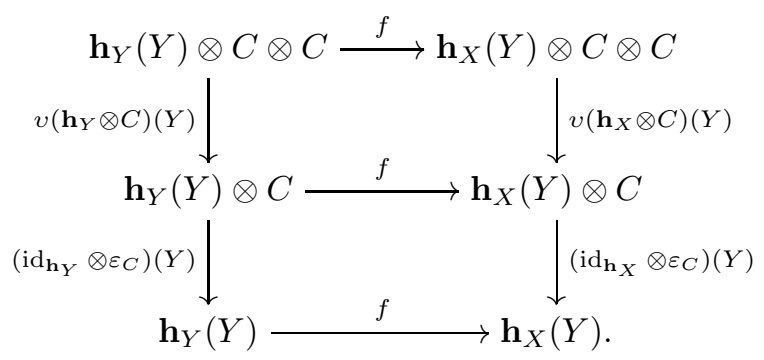

Thus, we have

$$
\begin{aligned}
f \circ\left(\left(\mathrm{id}_{\mathbf{h}_{Y}} \otimes \varepsilon_{C}\right) v\left(\mathbf{h}_{Y} \otimes C\right)\right)(Y)\left(\operatorname{id}_{Y} \otimes c \otimes d\right) \\
=\left(\left(\operatorname{id}_{\mathbf{h}_{X}} \otimes \varepsilon_{C}\right) v\left(\mathbf{h}_{X} \otimes C\right)\right)(Y)(f \otimes c \otimes d) .
\end{aligned}
$$

We now consider the morphism $\Psi_{Y}: C \otimes \mathbf{h}_{Y} \longrightarrow \mathbf{h}_{Y} \otimes C$ in $\mathscr{M}(\psi)_{\mathcal{D}}^{C}$ given by $\Psi_{Y}(X):=\psi_{X Y}$ as in Lemma 2.6. Then, using the naturality of $v: \mathscr{G} \mathscr{F} \longrightarrow 1_{\mathscr{M}(\psi)_{\mathcal{D}}^{C}}$ and $(2.2)$ we have the following commutative diagram:

$$
\begin{array}{ccc}
C \otimes \mathbf{h}_{Y}(Y) \otimes C \stackrel{\psi_{Y Y} \otimes \operatorname{id}_{C}}{\longrightarrow} & \mathbf{h}_{Y}(Y) \otimes C \otimes C \\
v\left(C \otimes \mathbf{h}_{Y}\right)(Y) \downarrow & \downarrow v\left(\mathbf{h}_{Y} \otimes C\right)(Y) \\
C \otimes \mathbf{h}_{Y}(Y) & \stackrel{\psi_{Y Y}}{\longrightarrow} & \mathbf{h}_{Y}(Y) \otimes C \\
\left(\varepsilon_{C} \otimes \operatorname{id}_{\mathbf{h}_{Y}}\right)(Y) \downarrow & \downarrow\left(\mathrm{id}_{\left.\mathbf{h}_{Y} \otimes \varepsilon_{C}\right)(Y)}\right. \\
\mathbf{h}_{Y}(Y) & \stackrel{\operatorname{id}_{\mathbf{h}_{Y}}(Y)}{\longrightarrow} & \mathbf{h}_{Y}(Y) .
\end{array}
$$


Using the fact that $\psi_{Y Y}\left(c \otimes \operatorname{id}_{Y}\right)=\operatorname{id}_{Y} \otimes c$, we now have

$$
\begin{aligned}
\left(\left(\mathrm{id}_{\mathbf{h}_{Y}} \otimes \varepsilon_{C}\right) v\left(\mathbf{h}_{Y} \otimes C\right)\right)(Y)\left(\mathrm{id}_{Y} \otimes c \otimes d\right) \\
=\left(\left(\varepsilon_{C} \otimes \mathrm{id}_{\mathbf{h}_{Y}}\right) v\left(C \otimes \mathbf{h}_{Y}\right)\right)(Y)\left(c \otimes \mathrm{id}_{Y} \otimes d\right) .
\end{aligned}
$$

Combining (3.7) and (3.8), we have

$$
\begin{aligned}
\left(\left(\operatorname{id}_{\mathbf{h}_{X}} \otimes \varepsilon_{C}\right)\right. & \left.v\left(\mathbf{h}_{X} \otimes C\right)\right)(Y)(f \otimes c \otimes d) \\
= & f \circ\left(\left(\varepsilon_{C} \otimes \operatorname{id}_{\mathbf{h}_{Y}}\right) v\left(C \otimes \mathbf{h}_{Y}\right)\right)(Y)\left(c \otimes \operatorname{id}_{Y} \otimes d\right) .
\end{aligned}
$$

By putting $Y=X$ and taking $f=\operatorname{id}_{X}$, the result of (3.6) is clear from (3.9).

Lemma 3.4: For any $v \in V$ and $Y \in \mathrm{Ob}(\mathcal{D})$, we have

$$
v\left(C \otimes C \otimes \mathbf{h}_{Y}\right)=\operatorname{id}_{C} \otimes v\left(C \otimes \mathbf{h}_{Y}\right)
$$

as a morphism of $\mathcal{D}$-modules.

Proof. For each $d \in C$, we define $\eta_{d}: C \otimes \mathbf{h}_{Y} \longrightarrow C \otimes C \otimes \mathbf{h}_{Y}$ by

$$
\eta_{d}(X)(c \otimes g):=d \otimes c \otimes g
$$

for each $X \in \operatorname{Ob}(\mathcal{D}), g \in \mathbf{h}_{Y}(X)$ and $c \in C$. It may be easily verified that $\eta_{d}$ is a morphism of right $\mathcal{D}$-modules. We now verify that

$$
\eta_{d}(X): C \otimes \mathbf{h}_{Y}(X) \longrightarrow C \otimes C \otimes \mathbf{h}_{Y}(X)
$$

is right $C$-colinear. We have

$$
\begin{aligned}
\sigma_{C \otimes C \otimes \mathbf{h}_{Y}(X)}^{r} & \left(\eta_{d}(X)(c \otimes g)\right) \\
& =\sigma_{C \otimes C \otimes \mathbf{h}_{Y}(X)}^{r}(d \otimes c \otimes g)=(d \otimes c)_{0} \otimes g_{\psi} \otimes(d \otimes c)_{1}{ }^{\psi} \\
& =d \otimes c_{1} \otimes g_{\psi} \otimes c_{2}{ }^{\psi}=\left(\eta_{d}(X) \otimes \operatorname{id}_{C}\right)\left(c_{1} \otimes g_{\psi} \otimes c_{2}{ }^{\psi}\right) \\
& =\left(\eta_{d}(X) \otimes \operatorname{id}_{C}\right) \sigma_{C \otimes \mathbf{h}_{Y}(X)}^{r}(c \otimes g) .
\end{aligned}
$$

Thus, $\eta_{d}: C \otimes \mathbf{h}_{Y} \longrightarrow C \otimes C \otimes \mathbf{h}_{Y}$ is a morphism in $\mathscr{M}(\psi)_{\mathcal{D}}^{C}$. Therefore, using the naturality of $v$, we have the following commutative diagram:

$$
\begin{array}{ccc}
C \otimes \mathbf{h}_{Y}(X) \otimes C & \stackrel{v\left(C \otimes \mathbf{h}_{Y}\right)(X)}{\longrightarrow} & C \otimes \mathbf{h}_{Y}(X) \\
\eta_{d}(X) \otimes \operatorname{id}_{C} \downarrow & \downarrow \eta_{d}(X) \\
C \otimes C \otimes \mathbf{h}_{Y}(X) \otimes C \stackrel{v\left(C \otimes C \otimes \mathbf{h}_{Y}\right)(X)}{\longrightarrow} & C \otimes C \otimes \mathbf{h}_{Y}(X) .
\end{array}
$$


Thus, for any $g \in \operatorname{Hom}_{\mathcal{D}}(X, Y)$ and $c, c^{\prime} \in C$, we get

$$
\begin{aligned}
v\left(C \otimes C \otimes \mathbf{h}_{Y}\right) & (X)\left(d \otimes c \otimes g \otimes c^{\prime}\right) \\
& =\left(v\left(C \otimes C \otimes \mathbf{h}_{Y}\right)\left(\eta_{d} \otimes \operatorname{id}_{C}\right)\right)(X)\left(c \otimes g \otimes c^{\prime}\right) \\
& =\left(\eta_{d} \circ v\left(C \otimes \mathbf{h}_{Y}\right)\right)(X)\left(c \otimes g \otimes c^{\prime}\right) \\
& =d \otimes v\left(C \otimes \mathbf{h}_{Y}\right)(X)\left(c \otimes g \otimes c^{\prime}\right) \\
& =\left(\operatorname{id}_{C} \otimes v\left(C \otimes \mathbf{h}_{Y}\right)\right)(X)\left(d \otimes c \otimes g \otimes c^{\prime}\right) .
\end{aligned}
$$

The result follows.

We now proceed to give another interpretation of $V=\operatorname{Nat}\left(\mathscr{G} \mathscr{F}, 1_{\mathscr{M}(\psi)_{\mathcal{D}}^{C}}\right)$. We consider a collection $\theta:=\left\{\theta_{X}: C \otimes C \longrightarrow \operatorname{End}_{\mathcal{D}}(X)\right\}_{X \in \mathrm{Ob}(\mathcal{D})}$ of $K$-linear maps satisfying the following conditions:

$$
\begin{aligned}
\left(\theta_{X}(c \otimes d)\right) \circ f & =f_{\psi_{\psi}} \circ \theta_{Y}\left(c^{\psi} \otimes d^{\psi}\right), \\
\theta_{X}\left(c \otimes d_{1}\right) \otimes d_{2} & =\left(\theta_{X}\left(c_{2} \otimes d\right)\right)_{\psi} \otimes c_{1}{ }^{\psi},
\end{aligned}
$$

for any $f \in \operatorname{Hom}_{\mathcal{D}}(Y, X)$. Let $V_{1}$ be the $K$-space consisting of all such $\theta$.

Proposition 3.5: Let $v \in V=\operatorname{Nat}\left(\mathscr{G} \mathscr{F}, 1_{\mathscr{M}(\psi)_{\mathcal{D}}^{C}}\right)$. For each $X \in \operatorname{Ob}(\mathcal{D})$, we define a $K$-linear map

$\theta_{X}: C \otimes C \longrightarrow \operatorname{End}_{\mathcal{D}}(X), \quad c \otimes d \mapsto\left(\left(\operatorname{id}_{\mathbf{h}_{X}} \otimes \varepsilon_{C}\right) v\left(\mathbf{h}_{X} \otimes C\right)\right)(X)\left(\operatorname{id}_{X} \otimes c \otimes d\right)$. Then, $\theta:=\left\{\theta_{X}\right\}_{X \in \mathrm{Ob}(\mathcal{D})}$ is an element in $V_{1}$.

Proof. Since $\operatorname{id}_{\mathbf{h}_{X}} \otimes \varepsilon_{C}: \mathbf{h}_{X} \otimes C \longrightarrow \mathbf{h}_{X}$ is a morphism of right $\mathcal{D}$-modules, we have

$$
\left(\theta_{X}(c \otimes d)\right) \circ f=\left(\operatorname{id}_{\mathbf{h}_{X}} \otimes \varepsilon_{C}\right)(Y)\left(v\left(\mathbf{h}_{X} \otimes C\right)(X)\left(\operatorname{id}_{X} \otimes c \otimes d\right) \cdot f\right)
$$

for $f \in \operatorname{Hom}_{\mathcal{D}}(Y, X)$ and $c, d \in C$. Since $v\left(\mathbf{h}_{X} \otimes C\right): \mathbf{h}_{X} \otimes C \otimes C \longrightarrow \mathbf{h}_{X} \otimes C$ is a morphism of right $\mathcal{D}$-modules, we also have

$$
\begin{aligned}
\left(v\left(\mathbf{h}_{X} \otimes C\right)(X)\right. & \left.\left(\operatorname{id}_{X} \otimes c \otimes d\right)\right) \cdot f \\
& =v\left(\mathbf{h}_{X} \otimes C\right)(Y)\left(\left(\operatorname{id}_{X} \otimes c \otimes d\right) \cdot f\right) \\
& =v\left(\mathbf{h}_{X} \otimes C\right)(Y)\left(\left(\mathbf{h}_{X} \otimes C \otimes C\right)(f)\left(\operatorname{id}_{X} \otimes c \otimes d\right)\right) \\
& =v\left(\mathbf{h}_{X} \otimes C\right)(Y)\left(\left(\mathbf{h}_{X} \otimes C\right)\left(f_{\psi}\right)\left(\operatorname{id}_{X} \otimes c\right) \otimes d^{\psi}\right) \\
& =v\left(\mathbf{h}_{X} \otimes C\right)(Y)\left(\mathbf{h}_{X}\left(f_{\psi_{\psi}}\right)\left(\operatorname{id}_{X}\right) \otimes c^{\psi} \otimes d^{\psi}\right) \\
& =v\left(\mathbf{h}_{X} \otimes C\right)(Y)\left(f_{\psi_{\psi}} \otimes c^{\psi} \otimes d^{\psi}\right) .
\end{aligned}
$$


The morphism $f_{\psi_{\psi}}: Y \longrightarrow X$ in $\mathcal{D}$ induces morphisms $\mathbf{h}_{Y} \otimes C \longrightarrow \mathbf{h}_{X} \otimes C$ and $\mathbf{h}_{Y} \otimes C \otimes C \longrightarrow \mathbf{h}_{X} \otimes C \otimes C$ in $\mathscr{M}(\psi)_{\mathcal{D}}^{C}$. Therefore, we have

$$
\begin{aligned}
\left(\theta_{X}(c \otimes d)\right) \circ f & =\left(\operatorname{id}_{\mathbf{h}_{X}} \otimes \varepsilon_{C}\right)(Y)\left(v\left(\mathbf{h}_{X} \otimes C\right)(X)\left(\operatorname{id}_{X} \otimes c \otimes d\right) \cdot f\right) \\
& =\left(\left(\operatorname{id}_{\mathbf{h}_{X}} \otimes \varepsilon_{C}\right) v\left(\mathbf{h}_{X} \otimes C\right)\right)(Y)\left(f_{\psi_{\psi}} \otimes c^{\psi} \otimes d^{\psi}\right) \\
& =f_{\psi_{\psi}} \circ\left(\left(\operatorname{id}_{\mathbf{h}_{Y}} \otimes \varepsilon_{C}\right) v\left(\mathbf{h}_{Y} \otimes C\right)\right)(Y)\left(\operatorname{id}_{Y} \otimes c^{\psi} \otimes d^{\psi}\right) \\
& =f_{\psi_{\psi}} \circ \theta_{Y}\left(c^{\psi} \otimes d^{\psi}\right) .
\end{aligned}
$$

This proves (3.11). We now verify that $\theta$ satisfies (3.12). Using Lemma 2.5, we know that $C \otimes \mathbf{h}_{Y}$ and $C \otimes C \otimes \mathbf{h}_{Y}$ belong to $\mathscr{M}(\psi)_{\mathcal{D}}^{C}$ for each $Y \in \operatorname{Ob}(\mathcal{D})$. For each $X \in \operatorname{Ob}(\mathcal{D})$, it may be easily seen that $C \otimes \mathbf{h}_{Y}(X)$ is also a left $C$-comodule with coaction given by

$$
\rho_{Y}^{l}(X):=\Delta_{C} \otimes \operatorname{id}_{\mathbf{h}_{Y}(X)} .
$$

Moreover, it may be easily verified that the following diagram commutes:

$$
\begin{array}{cr}
C \otimes \mathbf{h}_{Y}(X) \quad \stackrel{\rho_{Y}^{l}(X)}{\longrightarrow} & C \otimes C \otimes \mathbf{h}_{Y}(X) \\
\sigma_{C \otimes \mathbf{h}_{Y}(X)}^{r} \downarrow & \downarrow \sigma_{C \otimes C \otimes \mathbf{h}_{Y}(X)}^{r} \\
C \otimes \mathbf{h}_{Y}(X) \otimes C \stackrel{\rho_{Y}^{l}(X) \otimes \mathrm{id}}{\longrightarrow} C \otimes C \otimes \mathbf{h}_{Y}(X) \otimes C .
\end{array}
$$

This shows that $\rho_{Y}^{l}(X)$ is a morphism of right $C$-comodules. Further, for any $g \in \operatorname{Hom}_{\mathcal{D}}\left(X, X^{\prime}\right)$, we have the following commutative diagram:

$$
\begin{gathered}
C \otimes \mathbf{h}_{Y}\left(X^{\prime}\right) \stackrel{\rho_{Y}^{l}\left(X^{\prime}\right)}{\longrightarrow} C \otimes C \otimes \mathbf{h}_{Y}\left(X^{\prime}\right) \\
\qquad\left(C \otimes \mathbf{h}_{Y}\right)(g) \downarrow \\
C \otimes \mathbf{h}_{Y}(X) \stackrel{\rho_{Y}^{l}(X)}{\longrightarrow} C \otimes C \otimes \mathbf{h}_{Y}(X) .
\end{gathered}
$$

Thus, $\rho_{Y}^{l}: C \otimes \mathbf{h}_{Y} \longrightarrow C \otimes C \otimes \mathbf{h}_{Y}$ is a morphism of right $\mathcal{D}$-modules. This shows that $\rho_{Y}^{l}$ is a morphism in the category $\mathscr{M}(\psi)_{\mathcal{D}}^{C}$. Therefore, using the naturality of $v$ and Lemma 3.4, we have the following commutative diagram:

$$
\begin{array}{cc}
C \otimes \mathbf{h}_{Y}(X) \otimes C & C \otimes \mathbf{h}_{Y}(X) \\
\rho_{Y}^{l}(X) \otimes \mathrm{id}_{C} \downarrow & \downarrow \rho_{Y}^{l}(X) \\
C \otimes C \otimes \mathbf{h}_{Y}(X) \otimes C & \stackrel{v\left(C \otimes C \otimes \mathbf{h}_{Y}\right)(X)=\mathrm{id}_{C} \otimes v\left(C \otimes \mathbf{h}_{Y}\right)(X)}{\longrightarrow} C \otimes C \otimes \mathbf{h}_{Y}(X) .
\end{array}
$$


For any $c \otimes \operatorname{id}_{X} \otimes d \in C \otimes \mathbf{h}_{X}(X) \otimes C$, we set

$$
a_{i} \otimes f_{i}:=v\left(C \otimes \mathbf{h}_{X}\right)(X)\left(c \otimes \operatorname{id}_{X} \otimes d\right),
$$

Then, we have

$$
\rho_{X}^{l}(X)\left(a_{i} \otimes f_{i}\right)=a_{i 1} \otimes a_{i 2} \otimes f_{i}=\left(\operatorname{id}_{C} \otimes v\left(C \otimes \mathbf{h}_{X}\right)(X)\right)\left(c_{1} \otimes c_{2} \otimes \operatorname{id}_{X} \otimes d\right) .
$$

Now applying the map $\operatorname{id}_{C} \otimes \varepsilon_{C} \otimes \mathrm{id}_{\mathbf{h}_{X}}$ to both sides, we get

$$
\begin{aligned}
a_{i} \otimes f_{i} & =c_{1} \otimes\left(\left(\varepsilon_{C} \otimes \mathrm{id}_{\mathbf{h}_{X}}\right) v\left(C \otimes \mathbf{h}_{X}\right)\right)(X)\left(c_{2} \otimes \mathrm{id}_{X} \otimes d\right) \\
& =c_{1} \otimes\left(\left(\operatorname{id}_{\mathbf{h}_{X}} \otimes \varepsilon_{C}\right) v\left(\mathbf{h}_{X} \otimes C\right)\right)(X)\left(\mathrm{id}_{X} \otimes c_{2} \otimes d\right) \quad \text { (by Lemma 3.3) } \\
& =c_{1} \otimes \theta_{X}\left(c_{2} \otimes d\right) .
\end{aligned}
$$

Therefore, we have

$$
\psi\left(a_{i} \otimes f_{i}\right)=\left(\theta_{X}\left(c_{2} \otimes d\right)\right)_{\psi} \otimes c_{1}^{\psi} .
$$

Since $v\left(C \otimes \mathbf{h}_{Y}\right)(X)$ is a morphism of right $C$-comodules, we also have the following commutative diagram:

$$
\begin{array}{crc}
C \otimes \mathbf{h}_{Y}(X) \otimes C & \stackrel{v\left(C \otimes \mathbf{h}_{Y}\right)(X)}{\longrightarrow} & C \otimes \mathbf{h}_{Y}(X) \\
\pi_{C \otimes \mathbf{h}_{Y}(X) \otimes C} \downarrow & \downarrow \sigma_{C \otimes \mathbf{h}_{Y}(X)}^{r} \\
C \otimes \mathbf{h}_{Y}(X) \otimes C \otimes C \stackrel{v\left(C \otimes \mathbf{h}_{Y}\right)(X) \otimes \mathrm{id}_{C}}{\longrightarrow} & C \otimes \mathbf{h}_{Y}(X) \otimes C
\end{array}
$$

Thus, we have

$$
\begin{aligned}
\sigma_{C \otimes \mathbf{h}_{X}(X)}^{r}\left(a_{i} \otimes f_{i}\right) & =a_{i 1} \otimes f_{i \psi} \otimes a_{i 2} \psi \\
& =\left(v\left(C \otimes \mathbf{h}_{X}\right)(X) \otimes \operatorname{id}_{C}\right)\left(c \otimes \operatorname{id}_{X} \otimes d_{1} \otimes d_{2}\right) .
\end{aligned}
$$

Now, applying the map $\varepsilon_{C} \otimes \operatorname{id}_{\mathbf{h}_{X}} \otimes \mathrm{id}_{C}$ to both sides, we get

$$
\begin{aligned}
\varepsilon_{C}\left(a_{i 1}\right) & \left(f_{i \psi} \otimes a_{i 2}^{\psi}\right) \\
& =\left(\left(\varepsilon_{C} \otimes \mathrm{id}_{\mathbf{h}_{X}}\right) v\left(C \otimes \mathbf{h}_{X}\right)\right)(X)\left(c \otimes \mathrm{id}_{X} \otimes d_{1}\right) \otimes d_{2} \\
& =\left(\left(\operatorname{id}_{\mathbf{h}_{X}} \otimes \varepsilon_{C}\right) v\left(\mathbf{h}_{X} \otimes C\right)\right)(X)\left(\mathrm{id}_{X} \otimes c \otimes d_{1}\right) \otimes d_{2} \quad \text { (by Lemma 3.3). }
\end{aligned}
$$

Therefore,

$$
\psi\left(a_{i} \otimes f_{i}\right)=\theta_{X}\left(c \otimes d_{1}\right) \otimes d_{2} .
$$

It now follows from (3.15) and (3.16) that $\theta$ satisfies (3.12). 
Proposition 3.6: Let $\theta \in V_{1}$. Then, we have an element $v \in \operatorname{Nat}\left(\mathscr{G} \mathscr{F}, 1_{\mathscr{M}(\psi)_{\mathcal{D}}^{C}}\right)$ defined by

$$
v(\mathcal{M}): \mathcal{M} \otimes C \longrightarrow \mathcal{M}, \quad m \otimes c \mapsto \mathcal{M}\left(\theta_{X}\left(m_{1} \otimes c\right)\right)\left(m_{0}\right),
$$

for $\mathcal{M} \in \operatorname{Ob}\left(\mathscr{M}(\psi)_{\mathcal{D}}^{C}\right), X \in \operatorname{Ob}(\mathcal{D}), m \in \mathcal{M}(X)$ and $c \in C$.

Proof. We need to verify that $v(\mathcal{M}): \mathcal{M} \otimes C \longrightarrow \mathcal{M}$ is a morphism in $\mathscr{M}(\psi)_{\mathcal{D}}^{C}$ and that $v$ is indeed a natural transformation. We first verify that $v(\mathcal{M})$ is a morphism of right $\mathcal{D}$-modules. Let $f \in \operatorname{Hom}_{\mathcal{D}}(Y, X)$. Then, we have

$$
\begin{aligned}
\mathcal{M}(f) & (v(\mathcal{M})(X))(m \otimes c) \\
& =\mathcal{M}(f) \mathcal{M}\left(\theta_{X}\left(m_{1} \otimes c\right)\right)\left(m_{0}\right) \\
& =\mathcal{M}\left(\left(\theta_{X}\left(m_{1} \otimes c\right)\right) \circ f\right)\left(m_{0}\right) \\
& =\mathcal{M}\left(f_{\psi_{\psi}} \circ \theta_{Y}\left(m_{1}^{\psi} \otimes c^{\psi}\right)\right)\left(m_{0}\right) \\
& =\mathcal{M}\left(\theta_{Y}\left(m_{1}^{\psi} \otimes c^{\psi}\right)\right) \mathcal{M}\left(f_{\psi_{\psi}}\right)\left(m_{0}\right) \\
& =\mathcal{M}\left(\theta_{Y}\left(\left(\mathcal{M}\left(f_{\psi}\right)(m)\right)_{1} \otimes c^{\psi}\right)\right)\left(\mathcal{M}\left(f_{\psi}\right)(m)\right)_{0} \\
& =v(\mathcal{M})(Y)\left(\mathcal{M}\left(f_{\psi}\right)(m) \otimes c^{\psi}\right) \\
& =v(\mathcal{M})(Y)(\mathcal{M} \otimes C)(f)(m \otimes c) .
\end{aligned}
$$

We now verify that $v(\mathcal{M})(X): \mathcal{M}(X) \otimes C \longrightarrow \mathcal{M}(X)$ is a morphism of right $C$-comodules for every $X \in \mathrm{Ob}(\mathcal{D})$. For each $m \otimes c \in \mathcal{M}(X) \otimes C$, we have

$$
\begin{aligned}
(v(\mathcal{M}) & \left.(X) \otimes \operatorname{id}_{C}\right) \pi^{r}(m \otimes c) \\
& =v(\mathcal{M})(X)\left(m \otimes c_{1}\right) \otimes c_{2} \\
& =\mathcal{M}\left(\theta_{X}\left(m_{1} \otimes c_{1}\right)\right)\left(m_{0}\right) \otimes c_{2} \\
& =\mathcal{M}\left(\left(\theta_{X}\left(\left(m_{1}\right)_{2} \otimes c\right)\right)_{\psi}\right)\left(m_{0}\right) \otimes\left(m_{1}\right)_{1}{ }^{\psi} \\
& =\mathcal{M}\left(\left(\theta_{X}\left(m_{1} \otimes c\right)\right)_{\psi}\right)\left(m_{0}\right)_{0} \otimes\left(m_{0}\right)_{1}{ }^{\psi} \\
& =\rho_{\mathcal{M}(X)}\left(\mathcal{M}\left(\theta_{X}\left(m_{1} \otimes c\right)\right)\left(m_{0}\right)\right) \\
& =\rho_{\mathcal{M}(X)}(v(\mathcal{M})(X)(m \otimes c)) .
\end{aligned}
$$

It remains to show that $v: \mathscr{G} \mathscr{F} \longrightarrow 1_{\mathscr{M}(\psi)_{\mathcal{D}}^{C}}$ is a natural transformation. Let $\eta: \mathcal{M} \longrightarrow \mathcal{N}$ be a morphism in $\mathscr{M}(\psi)_{\mathcal{D}}^{C}$. Then, for every $X \in \operatorname{Ob}(\mathcal{D})$ 
and $m \otimes c \in \mathcal{M}(X) \otimes C$, we have

$$
\begin{aligned}
(v(\mathcal{N})(\eta & \left.\left.\otimes \operatorname{id}_{C}\right)\right)(X)(m \otimes c) \\
& =v(\mathcal{N})(X)(\eta(X)(m) \otimes c) \\
& =\mathcal{N}\left(\theta_{X}\left((\eta(X)(m))_{1} \otimes c\right)\right)(\eta(X)(m))_{0} \\
& =\mathcal{N}\left(\theta_{X}\left(m_{1} \otimes c\right)\right) \eta(X)\left(m_{0}\right) \\
& =\eta(X) \mathcal{M}\left(\theta_{X}\left(m_{1} \otimes c\right)\right)\left(m_{0}\right) \\
& =\eta(X) v(\mathcal{M})(X)(m \otimes c) .
\end{aligned}
$$

This proves the result.

Proposition 3.7: The $K$-spaces $V=\operatorname{Nat}\left(\mathscr{G} \mathscr{F}, 1_{\mathscr{M}(\psi)_{\mathcal{D}}^{C}}\right)$ and $V_{1}$ are isomorphic.

Proof. We define $\alpha: V \longrightarrow V_{1}$ by setting $\alpha(v)=\theta$, where $\theta$ is the collection of $K$-linear maps $\left\{\theta_{X}: C \otimes C \longrightarrow \operatorname{End}_{\mathcal{D}}(X)\right\}_{X \in \mathrm{Ob}(\mathcal{D})}$ defined by

$$
\theta_{X}(c \otimes d):=\left(\left(\operatorname{id}_{\mathbf{h}_{X}} \otimes \varepsilon_{C}\right) v\left(\mathbf{h}_{X} \otimes C\right)\right)(X)\left(\operatorname{id}_{X} \otimes c \otimes d\right)
$$

for $c, d \in C$. Then, $\alpha$ is a well-defined map by Proposition 3.5. We also define $\beta: V_{1} \longrightarrow V$ by setting $\beta(\theta)=v$, where $v: \mathscr{G} \mathscr{F} \longrightarrow 1_{\mathscr{M}(\psi)_{\mathcal{D}}^{C}}$ is defined by

$$
v(\mathcal{M}): \mathcal{M} \otimes C \longrightarrow \mathcal{M}, \quad m \otimes c \mapsto \mathcal{M}\left(\theta_{X}\left(m_{1} \otimes c\right)\right)\left(m_{0}\right)
$$

for $\mathcal{M} \in \mathrm{Ob}\left(\mathscr{M}(\psi)_{\mathcal{D}}^{C}\right), X \in \mathrm{Ob}(\mathcal{D}), m \otimes c \in \mathcal{M}(X) \otimes C$. By Proposition 3.6, $\beta$ is well-defined. We will now verify that $\alpha$ and $\beta$ are inverses of each other. Let $\theta \in V_{1}$. Then, for any $X, Y \in \mathrm{Ob}(\mathcal{D})$, we have

$$
\begin{aligned}
& (\alpha \beta(\theta))_{X}(c \otimes d) \\
& \quad=\left(\operatorname{id}_{\mathbf{h}_{X}} \otimes \varepsilon_{C}\right)(X)\left(\beta(\theta)\left(\mathbf{h}_{X} \otimes C\right)(X)\left(\mathrm{id}_{X} \otimes c \otimes d\right)\right) \\
& \quad=\left(\operatorname{id}_{\mathbf{h}_{X}} \otimes \varepsilon_{C}\right)(X)\left(\mathbf{h}_{X} \otimes C\right)\left(\theta_{X}\left(\left(\operatorname{id}_{X} \otimes c\right)_{1} \otimes d\right)\right)\left(\operatorname{id}_{X} \otimes c\right)_{0} \\
& \quad=\left(\operatorname{id}_{\mathbf{h}_{X}} \otimes \varepsilon_{C}\right)(X)\left(\mathbf{h}_{X} \otimes C\right)\left(\theta_{X}\left(c_{2} \otimes d\right)\right)\left(\operatorname{id}_{X} \otimes c_{1}\right) \\
& \quad=\left(\operatorname{id}_{\mathbf{h}_{X}} \otimes \varepsilon_{C}\right)(X)\left(\mathbf{h}_{X}\left(\left(\theta_{X}\left(c_{2} \otimes d\right)\right)_{\psi}\right)\left(\mathrm{id}_{X}\right) \otimes c_{1} \psi\right) \\
& \quad=\left(\operatorname{id}_{\mathbf{h}_{X}} \otimes \varepsilon_{C}\right)(X)\left(\mathbf{h}_{X}\left(\theta_{X}\left(c \otimes d_{1}\right)\right)\left(\mathrm{id}_{X}\right) \otimes d_{2}\right) \\
& \quad=\left(\theta_{X}\left(c \otimes d_{1}\right)\right) \varepsilon_{C}\left(d_{2}\right)=\theta_{X}(c \otimes d) .
\end{aligned}
$$

This proves that $(\alpha \beta(\theta))_{X}=\theta_{X}$ for all $X \in \mathrm{Ob}(\mathcal{D})$. Therefore, $(\alpha \beta)(\theta)=\theta$. For any $v \in V$, we now verify that $(\beta \alpha)(v)=v$. We set $\theta=\alpha(v)$. Then, by 
definition we have

$$
\begin{aligned}
(\beta \alpha)(v)(\mathcal{M}) & (X)(m \otimes c) \\
& =((\beta(\theta))(\mathcal{M}))(X)(m \otimes c) \\
& =\mathcal{M}\left(\theta_{X}\left(m_{1} \otimes c\right)\right)\left(m_{0}\right) \\
& =\mathcal{M}\left(\left(\operatorname{id}_{\mathbf{h}_{X}} \otimes \varepsilon_{C}\right)(X) v\left(\mathbf{h}_{X} \otimes C\right)(X)\left(\operatorname{id}_{X} \otimes m_{1} \otimes c\right)\right)\left(m_{0}\right) .
\end{aligned}
$$

For any $m^{\prime} \in \mathcal{M}(X)$, it may be easily verified that $\eta_{m^{\prime}}: \mathbf{h}_{X} \longrightarrow \mathcal{M}$ defined by

$$
\eta_{m^{\prime}}(Y)(f):=\mathcal{M}(f)\left(m^{\prime}\right)
$$

for each $f \in \operatorname{Hom}_{\mathcal{D}}(Y, X)$ is a morphism in Mod - D. By Lemma 2.4, this induces the morphism $\eta_{m^{\prime}} \otimes \operatorname{id}_{C}: \mathbf{h}_{X} \otimes C \longrightarrow \mathcal{M} \otimes C$ in $\mathscr{M}(\psi)_{\mathcal{D}}^{C}$ defined by

$$
\left(\eta_{m^{\prime}} \otimes \operatorname{id}_{C}\right)(Y)(f \otimes c):=\mathcal{M}(f)\left(m^{\prime}\right) \otimes c
$$

for $f \in \operatorname{Hom}_{\mathcal{D}}(Y, X)$ and $c \in C$. Since $v$ is a natural transformation, it follows easily that the following diagram commutes:

$$
\begin{array}{ccc}
\mathbf{h}_{X}(X) \otimes C \otimes C & \stackrel{\eta_{m^{\prime}}(X) \otimes \operatorname{id}_{C} \otimes \operatorname{id}_{C}}{\longrightarrow} & \mathcal{M}(X) \otimes C \otimes C \\
v\left(\mathbf{h}_{X} \otimes C\right)(X) \downarrow & & \downarrow v(\mathcal{M} \otimes C)(X) \\
\mathbf{h}_{X}(X) \otimes C & \stackrel{\eta_{m^{\prime}}(X) \otimes \operatorname{id}_{C}}{\longrightarrow} & \mathcal{M}(X) \otimes C \\
\left(\mathrm{id}_{\mathbf{h}_{X}} \otimes \varepsilon_{C}\right)(X) \downarrow & & \downarrow\left(\mathrm{id} \mathcal{M} \otimes \varepsilon_{C}\right)(X) \\
\mathbf{h}_{X}(X) & \stackrel{\eta_{m^{\prime}}(X)}{\longrightarrow} & \mathcal{M}(X) .
\end{array}
$$

In particular, we have

$$
\begin{aligned}
\mathcal{M}\left(\left(\left(\mathrm{id}_{\mathbf{h}_{X}} \otimes \varepsilon_{C}\right)\right.\right. & \left.\left.v\left(\mathbf{h}_{X} \otimes C\right)\right)(X)\left(\operatorname{id}_{X} \otimes m_{1} \otimes c\right)\right)\left(m_{0}\right) \\
= & \left(\left(\operatorname{id}_{\mathcal{M}} \otimes \varepsilon_{C}\right) v(\mathcal{M} \otimes C)\right)(X)\left(m_{0} \otimes m_{1} \otimes c\right) .
\end{aligned}
$$

The comodule structure on entwined modules determines a morphism in $\mathscr{M}(\psi)_{\mathcal{D}}^{C}$ as follows. We define $\tilde{\rho}: \mathcal{M} \longrightarrow \mathcal{M} \otimes C$ given by

$$
\tilde{\rho}(X):=\rho_{\mathcal{M}(X)}: \mathcal{M}(X) \longrightarrow \mathcal{M}(X) \otimes C
$$

for any $\mathcal{M} \in \operatorname{Ob}\left(\mathscr{M}(\psi)_{\mathcal{D}}^{C}\right)$ and $X \in \mathrm{Ob}(\mathcal{D})$. We first verify that $\tilde{\rho}$ is a morphism of right $\mathcal{D}$-modules. For any $f \in \operatorname{Hom}_{\mathcal{D}}(Y, X)$ and $m \in \mathcal{M}(X)$, we have

$$
\begin{aligned}
(\mathcal{M} \otimes C)(f)(\tilde{\rho}(X)(m)) & =(\mathcal{M} \otimes C)(f)\left(m_{0} \otimes m_{1}\right) \\
& =\mathcal{M}\left(f_{\psi}\right)\left(m_{0}\right) \otimes m_{1} \psi \\
& =\rho_{\mathcal{M}(Y)}(\mathcal{M}(f)(m))=\tilde{\rho}(Y)(\mathcal{M}(f)(m))
\end{aligned}
$$


It may be verified easily that $\tilde{\rho}(X): \mathcal{M}(X) \longrightarrow \mathcal{M}(X) \otimes C$ is right $C$-colinear. Thus, $\tilde{\rho}: \mathcal{M} \longrightarrow \mathcal{M} \otimes C$ is a morphism in $\mathscr{M}(\psi)_{\mathcal{D}}^{C}$. Therefore, we have the following commutative diagram:

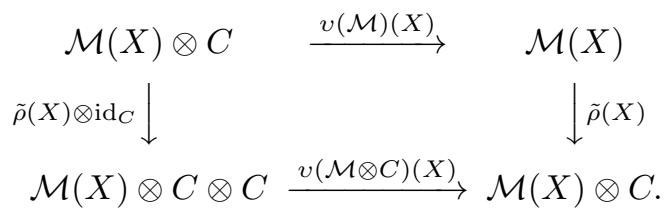

Thus, we get

$$
\begin{aligned}
v(\mathcal{M} \otimes C)(X)\left(\left(\tilde{\rho}(X) \otimes \operatorname{id}_{C}\right)(m \otimes c)\right) & =v(\mathcal{M} \otimes C)(X)\left(m_{0} \otimes m_{1} \otimes c\right) \\
& =\tilde{\rho}(X)(v(\mathcal{M})(X)(m \otimes c)) \\
& =\rho_{\mathcal{M}(X)}(v(\mathcal{M})(X)(m \otimes c)) .
\end{aligned}
$$

Now applying $\operatorname{id}_{\mathcal{M}(X)} \otimes \varepsilon_{C}$ on both sides, we obtain

$$
\begin{aligned}
(\beta \alpha)(v)(\mathcal{M})(X)(m \otimes c) & =\left(\operatorname{id}_{\mathcal{M}} \otimes \varepsilon_{C}\right)(X) v(\mathcal{M} \otimes C)(X)\left(m_{0} \otimes m_{1} \otimes c\right) \\
& =v(\mathcal{M})(X)(m \otimes c) .
\end{aligned}
$$

Theorem 3.8: Let $\mathscr{F}: \mathscr{M}(\psi)_{\mathcal{D}}^{C} \longrightarrow$ Mod-D be the forgetful functor and

$$
\mathscr{G}: \operatorname{Mod}-\mathcal{D} \longrightarrow \mathscr{M}(\psi)_{\mathcal{D}}^{C}, \quad \mathcal{N} \mapsto \mathcal{N} \otimes C
$$

be its right adjoint. Then, $\mathscr{F}$ is separable if and only if there exists $\theta \in V_{1}$ such that

$$
\theta_{X} \circ \Delta_{C}=\varepsilon_{C} \cdot \operatorname{id}_{X} \quad \forall X \in \operatorname{Ob}(\mathcal{D}) .
$$

Proof. We first recall from (3.1) that the unit of the adjunction is given by

$$
\mu(\mathcal{M}): \mathcal{M} \longrightarrow \mathcal{M} \otimes C, \quad \mu(\mathcal{M})(X)(m)=m_{0} \otimes m_{1},
$$

for $\mathcal{M} \in \operatorname{Ob}\left(\mathscr{M}(\psi)_{\mathcal{D}}^{C}\right)$ and $m \in \mathcal{M}(X)$. Suppose that $\mathscr{F}$ is separable. Then, by Theorem 3.2, there exists $v \in V$ such that $v \circ \mu=1_{\mathscr{M}(\psi)_{\mathcal{D}}^{C}}$. Therefore, using Proposition 3.7, corresponding to $v \in V$ we can obtain an element $\theta \in V_{1}$ given by

$$
\theta_{X}(c \otimes d)=\left(\left(\mathrm{id}_{\mathbf{h}_{X}} \otimes \varepsilon_{C}\right) v\left(\mathbf{h}_{X} \otimes C\right)\right)(X)\left(\mathrm{id}_{X} \otimes c \otimes d\right)
$$


for each $c, d \in C$. Moreover, we have

$$
\begin{aligned}
\left(\theta_{X} \circ \Delta_{C}\right)(c) & =\left(\left(\operatorname{id}_{\mathbf{h}_{X}} \otimes \varepsilon_{C}\right) v\left(\mathbf{h}_{X} \otimes C\right)\right)(X)\left(\operatorname{id}_{X} \otimes c_{1} \otimes c_{2}\right) \\
& =\left(\left(\operatorname{id}_{\mathbf{h}_{X}} \otimes \varepsilon_{C}\right) v\left(\mathbf{h}_{X} \otimes C\right)\right)(X)\left(\mu\left(\mathbf{h}_{X} \otimes C\right)(X)\right)\left(\operatorname{id}_{X} \otimes c\right) \\
& =\left(\left(\operatorname{id}_{\mathbf{h}_{X}} \otimes \varepsilon_{C}\right)\left(\operatorname{id}_{\mathbf{h}_{X} \otimes C}\right)\right)(X)\left(\operatorname{id}_{X} \otimes c\right) \\
& =\left(\operatorname{id}_{\mathbf{h}_{X}}(X) \otimes \varepsilon_{C}\right)\left(\operatorname{id}_{X} \otimes c\right)=\varepsilon_{C}(c) \operatorname{id}_{X}
\end{aligned}
$$

for any $c \in C$. Conversely, suppose that $\theta \in V_{1}$ is such that

$$
\theta_{X} \circ \Delta_{C}=\varepsilon_{C} \cdot \operatorname{id}_{X}
$$

for every $X \in \operatorname{Ob}(\mathcal{D})$. Corresponding to $\theta \in V_{1}$ there exists $v \in V$ defined by

$$
v(\mathcal{M}): \mathcal{M} \otimes C \longrightarrow \mathcal{M}, \quad m \otimes c \mapsto \mathcal{M}\left(\theta_{X}\left(m_{1} \otimes c\right)\right)\left(m_{0}\right),
$$

for $\mathcal{M} \in \operatorname{Ob}\left(\mathscr{M}(\psi)_{\mathcal{D}}^{C}\right), X \in \operatorname{Ob}(\mathcal{D}), m \in \mathcal{M}(X)$ and $c \in C$. Further, we have

$$
\begin{aligned}
(v \circ \mu)(\mathcal{M})(X)(m) & =v(\mathcal{M})(X)(\mu(\mathcal{M})(X)(m)) \\
& =v(\mathcal{M})(X)\left(m_{0} \otimes m_{1}\right) \\
& =\mathcal{M}\left(\theta_{X}\left(\left(m_{0}\right)_{1} \otimes m_{1}\right)\right)\left(m_{0}\right)_{0} \\
& =\mathcal{M}\left(\theta_{X}\left(\left(m_{1}\right)_{1} \otimes\left(m_{1}\right)_{2}\right)\right)\left(m_{0}\right) \\
& =\mathcal{M}\left(\left(\theta_{X} \circ \Delta_{C}\right)\left(m_{1}\right)\right)\left(m_{0}\right) \\
& =\mathcal{M}\left(\left(\operatorname{id}_{X}\right) \varepsilon_{C}\left(m_{1}\right)\right)\left(m_{0}\right)=m .
\end{aligned}
$$

This shows that $v \circ \mu=1_{\mathscr{M}(\psi)_{\mathcal{D}}^{C}}$. Hence, $\mathscr{F}$ is separable by Theorem 3.2.

Next we investigate the separability of the functor $\mathscr{G}: \operatorname{Mod}-\mathcal{D} \longrightarrow \mathscr{M}(\psi)_{\mathcal{D}}^{C}$ given by

$$
\mathscr{G}(\mathcal{N})=\mathcal{N} \otimes C
$$

for any $\mathcal{N} \in \operatorname{Mod}-\mathcal{D}$. Since $\mathscr{G}$ is a right adjoint of $\mathscr{F}$, it follows from Theorem 3.2 that the functor $\mathscr{G}$ is separable if and only if there exists a natural transformation $\omega: 1_{\mathrm{Mod}-\mathcal{D}} \longrightarrow \mathscr{F} \mathscr{G}$ such that

$$
\nu \circ \omega=1_{\mathrm{Mod}-\mathcal{D}}
$$

where $\nu$ is the counit of the adjunction as explained in (3.2). We set

$$
W:=\operatorname{Nat}\left(1_{\mathrm{Mod}-\mathcal{D}}, \mathscr{F} \mathscr{G}\right)
$$

and proceed to give another interpretation of $W$. 
We define $h: \mathcal{D}^{o p} \otimes \mathcal{D} \longrightarrow$ Vect $_{K}$ as

$$
h(X, Y):=\operatorname{Hom}_{\mathcal{D}}(X, Y), \quad(h(\phi))(f):=\phi^{\prime \prime} f \phi^{\prime},
$$

for any $(X, Y) \in \operatorname{Ob}\left(\mathcal{D}^{o p} \otimes \mathcal{D}\right), \phi:=\left(\phi^{\prime}, \phi^{\prime \prime}\right) \in \operatorname{Hom}_{\mathcal{D}^{o p} \otimes \mathcal{D}}\left((X, Y),\left(X^{\prime}, Y^{\prime}\right)\right)$ and $f \in \operatorname{Hom}_{\mathcal{D}}(X, Y)$. Similarly, we define the functor $h \otimes C: \mathcal{D}^{o p} \otimes \mathcal{D} \longrightarrow \operatorname{Vect}_{K}$ as

$$
\begin{aligned}
(h \otimes C)(X, Y) & :=\operatorname{Hom}_{\mathcal{D}}(X, Y) \otimes C, \\
((h \otimes C)(\phi))(f \otimes c) & :=\phi^{\prime \prime} f \phi_{\psi}^{\prime} \otimes c^{\psi}
\end{aligned}
$$

for any $(X, Y) \in \operatorname{Ob}\left(\mathcal{D}^{o p} \otimes \mathcal{D}\right), \phi:=\left(\phi^{\prime}, \phi^{\prime \prime}\right) \in \operatorname{Hom}_{\mathcal{D}}{ }^{o p} \otimes \mathcal{D}\left((X, Y),\left(X^{\prime}, Y^{\prime}\right)\right)$, $f \in \operatorname{Hom}_{\mathcal{D}}(X, Y)$ and $c \in C$. By slight abuse of notation, we will make no distinction between functors $\mathcal{D}^{o p} \otimes \mathcal{D} \longrightarrow$ Vect $_{K}$ and functors $\mathcal{D} \longrightarrow \operatorname{Mod}-\mathcal{D}$. We observe that $h \otimes C: \mathcal{D}^{o p} \otimes \mathcal{D} \longrightarrow$ Vect $_{K}$ corresponds to $\mathscr{F} \circ(\mathbf{h} \otimes C)$ when viewed as a functor from $\mathcal{D} \longrightarrow \operatorname{Mod}-\mathcal{D}$.

Given a natural transformation $\eta: h \longrightarrow h \otimes C$, it is easy to see that

$$
\eta(-, Y): \mathbf{h}_{Y}=\operatorname{Hom}_{\mathcal{D}}(-, Y) \longrightarrow \mathbf{h}_{Y} \otimes C=\operatorname{Hom}_{\mathcal{D}}(-, Y) \otimes C
$$

is a morphism of right $\mathcal{D}$-modules for each $Y \in \mathrm{Ob}(\mathcal{D})$. Similarly, for each $X \in \mathrm{Ob}(\mathcal{D})$,

$$
\eta(X,-):{ }_{X} \mathbf{h}=\operatorname{Hom}_{\mathcal{D}}(X,-) \longrightarrow{ }_{X} \mathbf{h} \otimes C=\operatorname{Hom}_{\mathcal{D}}(X,-) \otimes C
$$

is a morphism of left $\mathcal{D}$-modules.

Throughout the rest of this section, we set $W_{1}:=\operatorname{Nat}(h, h \otimes C)$, the $K$-space consisting of all natural transformations between the functors $h$ and $h \otimes C$.

Lemma 3.9: Let $\eta \in W_{1}$. We set $\eta(X, X)\left(\mathrm{id}_{X}\right)=\sum a_{X} \otimes c_{X}$ for each $X \in \mathrm{Ob}(\mathcal{D})$ and $\eta(Y, Z)(g):=\sum \hat{g} \otimes c_{g}$ for any $g \in \operatorname{Hom}_{\mathcal{D}}(Y, Z)$. Then,

$$
\eta(Y, Z)(g)=\sum \hat{g} \otimes c_{g}=\sum a_{Z} g_{\psi} \otimes c_{Z} \psi=\sum g a_{Y} \otimes c_{Y} .
$$

Proof. Since $\eta(-, Z): \mathbf{h}_{Z} \longrightarrow \mathbf{h}_{Z} \otimes C$ is a morphism of right $\mathcal{D}$-modules for each $Z \in \mathrm{Ob}(\mathcal{D})$, we have the following commutative diagram:

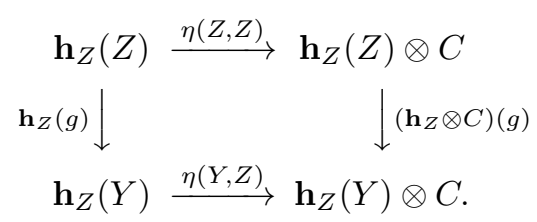


This diagram along with Lemma 2.4 gives

$$
\begin{aligned}
\eta(Y, Z)(g) & =\sum\left(\left(\mathbf{h}_{Z} \otimes C\right)(g)\right)\left(a_{Z} \otimes c_{Z}\right) \\
& =\sum \mathbf{h}_{Z}\left(g_{\psi}\right)\left(a_{Z}\right) \otimes c_{Z} \psi \\
& =\sum a_{Z} g_{\psi} \otimes c_{Z}{ }^{\psi} .
\end{aligned}
$$

Since $\eta(Y,-):{ }_{Y} \mathbf{h} \longrightarrow{ }_{Y} \mathbf{h} \otimes C$ is a morphism of left $\mathcal{D}$-modules, we also have the following commutative diagram:

$$
\begin{array}{cc}
Y \mathbf{h}(Y) \stackrel{\eta(Y, Y)}{\longrightarrow} \underset{Y}{ } \mathbf{h}(Y) \otimes C \\
Y \mathbf{h}(g) \downarrow & \downarrow{ }_{(Y \mathbf{h} \otimes C)(g)} \\
Y \mathbf{h}(Z) \stackrel{\eta(Y, Z)}{\longrightarrow} & \stackrel{h}{ } \mathbf{h}(Z) \otimes C
\end{array}
$$

This gives

$$
\eta(Y, Z)(g)=\left({ }_{Y} \mathbf{h} \otimes C\right)(g)\left(\sum a_{Y} \otimes c_{Y}\right)=\sum g a_{Y} \otimes c_{Y} .
$$

The result now follows from (3.22) and (3.23).

Proposition 3.10: The $K$-spaces

$$
W=\operatorname{Nat}\left(1_{\mathrm{Mod}-\mathcal{D}}, \mathscr{F} \mathscr{G}\right) \quad \text { and } \quad W_{1}=\operatorname{Nat}(h, h \otimes C)
$$

are isomorphic.

Proof. We define a $K$-linear map $\gamma: W \longrightarrow W_{1}$ by setting

$$
\eta=\gamma(\omega): h \longrightarrow h \otimes C, \quad \eta(X, Y):=\omega\left(\mathbf{h}_{Y}\right)(X),
$$

for any $(X, Y) \in \operatorname{Ob}\left(\mathcal{D}^{o p} \otimes \mathcal{D}\right)$. We now verify that the map is well-defined. Let

$$
\phi:=\left(\phi^{\prime}, \phi^{\prime \prime}\right) \in \operatorname{Hom}_{\mathcal{D}^{o p} \otimes \mathcal{D}}\left((X, Y),\left(X^{\prime}, Y^{\prime}\right)\right) .
$$

Since $\omega\left(\mathbf{h}_{Y}\right): \mathbf{h}_{Y} \longrightarrow \mathbf{h}_{Y} \otimes C$ is a morphism of right $\mathcal{D}$-modules, we have the following commutative diagram:

$$
\begin{array}{cc}
\mathbf{h}_{Y}(X) \stackrel{\omega\left(\mathbf{h}_{Y}\right)(X)}{\longrightarrow} & \mathbf{h}_{Y}(X) \otimes C \\
\mathbf{h}_{Y}\left(\phi^{\prime}\right) \downarrow & \downarrow\left(\mathbf{h}_{Y} \otimes C\right)\left(\phi^{\prime}\right) \\
\mathbf{h}_{Y}\left(X^{\prime}\right) \stackrel{\omega\left(\mathbf{h}_{Y}\right)\left(X^{\prime}\right)}{\longrightarrow} & \mathbf{h}_{Y}\left(X^{\prime}\right) \otimes C .
\end{array}
$$


The morphism $\phi^{\prime \prime}: Y \longrightarrow Y^{\prime}$ in $\mathcal{D}$ induces a morphism $\phi^{\prime \prime}: \mathbf{h}_{Y} \longrightarrow \mathbf{h}_{Y^{\prime}}$ of right $\mathcal{D}$-modules. Therefore, using the naturality of $\omega$, we get the following commutative diagram:

$$
\begin{array}{ccc}
\mathbf{h}_{Y}\left(X^{\prime}\right) \stackrel{\omega\left(\mathbf{h}_{Y}\right)\left(X^{\prime}\right)}{\longrightarrow} & \mathbf{h}_{Y}\left(X^{\prime}\right) \otimes C \\
X^{\prime} \mathbf{h}\left(\phi^{\prime \prime}\right)=\mathbf{h}_{\phi^{\prime \prime}}\left(X^{\prime}\right) \downarrow & \downarrow\left(X^{\prime} \mathbf{h}\left(\phi^{\prime \prime}\right) \otimes \operatorname{id}_{C}\right)=\left(\mathbf{h}_{\phi^{\prime \prime}}\left(X^{\prime}\right) \otimes \operatorname{id}_{C}\right) \\
\mathbf{h}_{Y^{\prime}}\left(X^{\prime}\right) \stackrel{\omega\left(\mathbf{h}_{Y^{\prime}}\right)\left(X^{\prime}\right)}{\longrightarrow} \mathbf{h}_{Y^{\prime}}\left(X^{\prime}\right) \otimes C .
\end{array}
$$

We now observe that for $f \in \operatorname{Hom}_{\mathcal{D}}(X, Y)$, we have

$$
\begin{aligned}
(h(\phi))(f) & =\phi^{\prime \prime} f \phi^{\prime}={ }_{X^{\prime}} \mathbf{h}\left(\phi^{\prime \prime}\right)\left(\mathbf{h}_{Y}\left(\phi^{\prime}\right)(f)\right), \\
((h \otimes C)(\phi))(g \otimes c) & =\phi^{\prime \prime} g \phi_{\psi}^{\prime} \otimes c^{\psi}=\left(X^{\prime} \mathbf{h}\left(\phi^{\prime \prime}\right) \otimes \operatorname{id}_{C}\right)\left(\left(\mathbf{h}_{Y} \otimes C\right)\left(\phi^{\prime}\right)(g \otimes c)\right) .
\end{aligned}
$$

Thus, by combining the diagrams (3.24) and (3.25), we obtain the following commutative diagram:

$$
\begin{array}{cr}
h(X, Y) \stackrel{\eta(X, Y)}{\longrightarrow} & h(X, Y) \otimes C \\
h(\phi) \downarrow & \downarrow(h \otimes C)(\phi) \\
h\left(X^{\prime}, Y^{\prime}\right) \stackrel{\eta\left(X^{\prime}, Y^{\prime}\right)}{\longrightarrow} & h\left(X^{\prime}, Y^{\prime}\right) \otimes C .
\end{array}
$$

This shows that $\eta \in W_{1}$.

Conversely, let $\eta \in W_{1}=\operatorname{Nat}(h, h \otimes C)$. For any $Y \in \operatorname{Ob}(\mathcal{D})$,

$$
\eta(-, Y): \mathbf{h}_{Y} \longrightarrow \mathbf{h}_{Y} \otimes C
$$

is a morphism of right $\mathcal{D}$-modules. For any $f \in \operatorname{Hom}_{\mathcal{D}}(X, Y)$, the naturality of $\eta$ gives us the following commutative diagram:

$$
\begin{aligned}
& \mathbf{h}_{X}=\operatorname{Hom}_{\mathcal{D}}(-, X) \stackrel{\eta(-, X)}{\longrightarrow} \operatorname{Hom}_{\mathcal{D}}(-, X) \otimes C=\mathbf{h}_{X} \otimes C \\
& h(-, f) \downarrow \downarrow h(-, f) \otimes \mathrm{id}_{C} \\
& \mathbf{h}_{Y}=\operatorname{Hom}_{\mathcal{D}}(-, Y) \stackrel{\eta(-, Y)}{\longrightarrow} \operatorname{Hom}_{\mathcal{D}}(-, Y) \otimes C=\mathbf{h}_{Y} \otimes C .
\end{aligned}
$$

Now, for any $\mathcal{M}$ in Mod- $\mathcal{D}$, we know that

$$
\mathcal{M}=\underset{y \in \operatorname{el}(\mathcal{M})}{\operatorname{colim}} \mathbf{h}_{|y|} .
$$

Similarly,

$$
\mathcal{M} \otimes C=\operatorname{colim}_{y \in \operatorname{el}(\mathcal{M})}\left(\mathbf{h}_{|y|} \otimes C\right)
$$


where the colimit is taken in Mod-D . Thus, the morphisms as in (3.26) induce a morphism $\omega(\mathcal{M}): \mathcal{M} \longrightarrow \mathcal{M} \otimes C$ of right $\mathcal{D}$-modules. Moreover, for any morphism $\mathcal{M} \stackrel{\zeta}{\longrightarrow} \mathcal{N}$ in Mod- $\mathcal{D}$, the commutative diagrams as in (3.27) induce the following equality:

$$
\left(\zeta \otimes \operatorname{id}_{C}\right) \circ \omega(\mathcal{M})=\omega(\mathcal{N}) \circ \zeta .
$$

Therefore, for $\eta \in W_{1}$ we have obtained a natural transformation

$$
\omega: 1_{\operatorname{Mod}-\mathcal{D}} \longrightarrow \mathscr{F} \mathscr{G}
$$

in $W$. We will denote this $K$-linear map by $\delta: W_{1} \longrightarrow W$, i.e., $\delta(\eta)=\omega$ determined by $\omega\left(\mathbf{h}_{Y}\right):=\eta(-, Y)$ for each $Y \in \operatorname{Ob}(\mathcal{D})$. It may be easily verified that the morphisms $\gamma$ and $\delta$ are inverses of each other.

Theorem 3.11: Let $\mathscr{F}: \mathscr{M}(\psi)_{\mathcal{D}}^{C} \longrightarrow$ Mod-D be the forgetful functor and $\mathscr{G}: \operatorname{Mod}-\mathcal{D} \longrightarrow \mathscr{M}(\psi)_{\mathcal{D}}^{C}, \mathcal{N} \mapsto \mathcal{N} \otimes C$ be its right adjoint. Then $\mathscr{G}$ is separable if and only if there exists $\eta \in W_{1}=\operatorname{Nat}(h, h \otimes C)$ such that

$$
\left(\operatorname{id}_{h} \otimes \varepsilon_{C}\right) \eta=\mathrm{id}_{h} .
$$

Proof. Suppose that $\mathscr{G}$ is separable. Then, by Theorem 3.2, there exists $\omega \in W=\operatorname{Nat}\left(1_{\text {Mod -D }}, \mathscr{F} \mathscr{G}\right)$ such that $\nu \circ \omega=1_{\text {Mod - } \mathcal{D}}$, where $\nu$ is the counit of the adjunction. Using Proposition 3.10, corresponding to $\omega \in W$, there exists an element $\eta \in W_{1}$ given by $\eta(X, Y)=\omega\left(\mathbf{h}_{Y}\right)(X)$ for every $(X, Y) \in \mathrm{Ob}\left(\mathcal{D}^{o p} \otimes \mathcal{D}\right)$. The condition (3.28) now follows from the definition of the counit in (3.2).

Conversely, let $\eta \in W_{1}$ be such that $\left(\operatorname{id}_{h} \otimes \varepsilon_{C}\right) \eta=\mathrm{id}_{h}$. We consider $\omega: 1_{\text {Mod }-\mathcal{D}} \longrightarrow \mathscr{F} \mathscr{G}$ given by $\omega\left(\mathbf{h}_{Y}\right):=\eta(-, Y)$ for each $Y \in \mathrm{Ob}(\mathcal{D})$. Then,

$$
\left(\mathrm{id}_{\mathbf{h}_{Y}} \otimes \varepsilon_{C}\right) \omega\left(\mathbf{h}_{Y}\right)=\left(\mathrm{id}_{\mathbf{h}_{Y}} \otimes \varepsilon_{C}\right) \eta(-, Y)=\mathrm{id}_{\mathbf{h}_{Y}} .
$$

Since $\mathscr{F}$ is a left adjoint and it is clear from the definition that $\mathscr{G}$ preserves colimits, we obtain that $\left(\operatorname{id}_{\mathcal{N}} \otimes \varepsilon_{C}\right) \omega(\mathcal{N})=\operatorname{id}_{\mathcal{N}}$ for any $\mathcal{N} \in \operatorname{Mod}-\mathcal{D}$, i.e.,

$$
\left(\mathrm{id} \otimes \varepsilon_{C}\right) \omega=1_{\text {Mod }-\mathcal{D}} .
$$

Therefore, $\mathscr{G}$ is separable by Theorem 3.2.

3.2. Frobenius conditions. Let $F: \mathcal{A} \longrightarrow \mathcal{B}$ be a functor which has a right adjoint $G: \mathcal{B} \longrightarrow \mathcal{A}$. Then, the pair $(F, G)$ is called a Frobenius pair if $G$ is both a right and a left adjoint of $F$. We recall the following characterization for Frobenius pairs (see $[8, \S 1]$ ): 
Theorem 3.12: Let $F: \mathcal{A} \longrightarrow \mathcal{B}$ be a functor which has a right adjoint $G$. Then, $(F, G)$ is a Frobenius pair if and only if there exist $v \in \operatorname{Nat}\left(G F, 1_{\mathcal{A}}\right)$ and $\omega \in \operatorname{Nat}\left(1_{\mathcal{B}}, F G\right)$ such that

$$
\begin{gathered}
F(v(M)) \circ \omega(F(M))=\operatorname{id}_{F(M)}, \\
v(G(N)) \circ G(\omega(N))=\operatorname{id}_{G(N),}
\end{gathered}
$$

for all $M \in \mathcal{A}$ and $N \in \mathcal{B}$.

Lemma 3.13: For any $\omega \in W=\operatorname{Nat}\left(1_{\operatorname{Mod}-\mathcal{D}}, \mathscr{F} \mathscr{G}\right), N \in \operatorname{Comod}-C$ and $Y \in \operatorname{Ob}(\mathcal{D})$, we have

$$
\omega\left(N \otimes \mathbf{h}_{Y}\right)=\operatorname{id}_{N} \otimes \omega\left(\mathbf{h}_{Y}\right) .
$$

Proof. For each $n \in N$, we define $\zeta_{n}: \mathbf{h}_{Y} \longrightarrow N \otimes \mathbf{h}_{Y}$ by

$$
\zeta_{n}(X)(f):=n \otimes f
$$

for any $X \in \operatorname{Ob}(\mathcal{D})$ and $f \in \operatorname{Hom}_{\mathcal{D}}(X, Y)$. It may be easily verified that $\zeta_{n}$ is a morphism of right $\mathcal{D}$-modules. Therefore, using the naturality of $\omega$, we have the following commutative diagram:

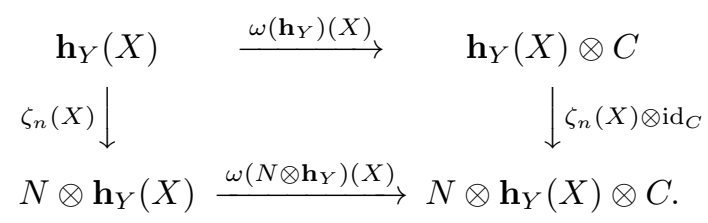

Let $f \in \operatorname{Hom}_{\mathcal{D}}(X, Y)$. We set

$$
\omega\left(\mathbf{h}_{Y}\right)(X)(f)=\sum \hat{f} \otimes c_{f} .
$$

Then, we have

$$
\begin{aligned}
\omega\left(N \otimes \mathbf{h}_{Y}\right)(X)(n \otimes f) & =\left(\zeta_{n}(X) \otimes \operatorname{id}_{C}\right) \omega\left(\mathbf{h}_{Y}\right)(X)(f) \\
& =\sum\left(\zeta_{n}(X) \otimes \operatorname{id}_{C}\right)\left(\hat{f} \otimes c_{f}\right) \\
& =\sum n \otimes \hat{f} \otimes c_{f} \\
& =\left(\operatorname{id}_{N} \otimes \omega\left(\mathbf{h}_{Y}\right)\right)(X)(n \otimes f) .
\end{aligned}
$$

The result follows. 
Theorem 3.14: Let $\mathscr{F}: \mathscr{M}(\psi)_{\mathcal{D}}^{C} \longrightarrow$ Mod-D be the forgetful functor and $\mathscr{G}: \operatorname{Mod}-\mathcal{D} \longrightarrow \mathscr{M}(\psi)_{\mathcal{D}}^{C}, \mathcal{N} \mapsto \mathcal{N} \otimes C$ be its right adjoint. Then, $(\mathscr{F}, \mathscr{G})$ is a Frobenius pair if and only if there exist $\theta \in V_{1}$ and $\eta \in W_{1}$ such that the following conditions hold:

$$
\begin{aligned}
& \varepsilon_{C}(d) f=\sum \hat{f} \circ \theta_{X}\left(c_{f} \otimes d\right), \\
& \varepsilon_{C}(d) f=\sum \hat{f}_{\psi} \circ \theta_{X}\left(d^{\psi} \otimes c_{f}\right),
\end{aligned}
$$

for any $f \in \operatorname{Hom}_{\mathcal{D}}(X, Y), d \in C$ and $\eta(X, Y)(f)=\sum \hat{f} \otimes c_{f}$.

Proof. Suppose there exist $\theta \in V_{1}$ and $\eta \in W_{1}$ such that (3.31) and (3.32) hold. Then, using the isomorphisms $V \cong V_{1}$ and $W \cong W_{1}$ as in Propositions 3.7 and 3.10, there exist $v \in V$ and $\omega \in W$ corresponding to $\theta \in V_{1}$ and $\eta \in W_{1}$ respectively. We also know by Proposition 2.9 that the collection

$$
\left\{N \otimes \mathbf{h}_{Y}\right\},
$$

where $N$ ranges over all (isomorphisms classes of) finite-dimensional $C$-comodules and $Y$ ranges over all objects in $\mathcal{D}$, forms a generating set for $\mathscr{M}(\psi)_{\mathcal{D}}^{C}$. Therefore, we first verify the condition (3.29) for

$$
\mathcal{M}=N \otimes \mathbf{h}_{Y} \in \mathscr{M}(\psi)_{\mathcal{D}}^{C},
$$

where $N \in \operatorname{Comod}-C$ and $Y \in \operatorname{Ob}(\mathcal{D})$. For any $n \otimes f \in N \otimes \operatorname{Hom}_{\mathcal{D}}(X, Y)$, we have

$$
\begin{aligned}
(\mathscr{F}( & \left.\left.v\left(N \otimes \mathbf{h}_{Y}\right)\right) \circ \omega\left(\mathscr{F}\left(N \otimes \mathbf{h}_{Y}\right)\right)\right)(X)(n \otimes f) \\
= & v\left(N \otimes \mathbf{h}_{Y}\right)(X)\left(\operatorname{id}_{N} \otimes \omega\left(\mathbf{h}_{Y}\right)\right)(X)(n \otimes f) \\
& =v\left(N \otimes \mathbf{h}_{Y}\right)(X)\left(\operatorname{id}_{N} \otimes \eta(X, Y)\right)(n \otimes f) \\
& =\sum v\left(N \otimes \mathbf{h}_{Y}\right)(X)\left(n \otimes \hat{f} \otimes c_{f}\right) \\
& =\sum\left(N \otimes h_{Y}\right)\left(\theta_{X}\left((n \otimes \hat{f})_{1} \otimes c_{f}\right)\right)(n \otimes \hat{f})_{0} \\
& =\sum\left(N \otimes h_{Y}\right)\left(\theta_{X}\left(n_{1} \psi \otimes c_{f}\right)\right)\left(n_{0} \otimes \hat{f}_{\psi}\right) \\
& =\sum n_{0} \otimes \hat{f}_{\psi} \circ \theta_{X}\left(n_{1} \psi \otimes c_{f}\right) \\
& =n_{0} \otimes \varepsilon_{C}\left(n_{1}\right) f \\
& =n \otimes f .
\end{aligned}
$$


This proves (3.29) for the generators of $\mathscr{M}(\psi)_{\mathcal{D}}^{C}$. As explained in the proof of Proposition 2.9 , for any $\mathcal{M}$ in $\mathscr{M}(\psi)_{\mathcal{D}}^{C}$, there is an epimorphism

$$
\bigoplus_{m \in \mathrm{el}(\mathcal{M})} \eta_{m}: \bigoplus_{m \in \mathrm{el}(\mathcal{M})} V_{m} \otimes \mathbf{h}_{|m|} \longrightarrow \mathcal{M}
$$

in $\mathscr{M}(\psi)_{\mathcal{D}}^{C}$. The morphism

$$
\eta:=\bigoplus_{m \in \mathrm{el}(\mathcal{M})} \eta_{m}
$$

induces the following commutative diagram:

$$
\begin{array}{ccc}
\bigoplus \mathscr{F}\left(V_{m} \otimes \mathbf{h}_{|m|}\right) & \stackrel{\oplus \mathscr{F}\left(v\left(V_{m} \otimes \mathbf{h}_{|m|}\right)\right) \omega\left(\mathscr{F}\left(V_{m} \otimes \mathbf{h}_{|m|}\right)\right)}{\longrightarrow} & \oplus \mathscr{F}\left(V_{m} \otimes \mathbf{h}_{|m|}\right) \\
\mathscr{F}(\eta) \downarrow & \downarrow \mathscr{F}(\eta) \\
\mathscr{F}(\mathcal{M}) & \stackrel{\mathscr{F}(v(\mathcal{M})) \omega(\mathscr{F}(\mathcal{M}))}{\longrightarrow} & \mathscr{F}(\mathcal{M}) .
\end{array}
$$

From (3.33), it follows that

$$
\mathscr{F}\left(v\left(V_{m} \otimes \mathbf{h}_{|m|}\right)\right) \omega\left(\mathscr{F}\left(V_{m} \otimes \mathbf{h}_{|m|}\right)\right)=\operatorname{id}{ }_{\mathscr{F}\left(V_{m} \otimes \mathbf{h}_{|m|}\right)}
$$

for each $m \in \operatorname{el}(\mathcal{M})$. Thus, by the commutative diagram (3.34), we have

$$
(\mathscr{F}(v(\mathcal{M})) \circ \omega(\mathscr{F}(\mathcal{M}))) \circ \mathscr{F}(\eta)=\mathscr{F}(\eta) .
$$

Since $\mathscr{F}$ is a left adjoint, it preserves epimorphisms. Since $\eta$ is an epimorphism, so is $\mathscr{F}(\eta)$. Therefore, (3.35) implies that

$$
\mathscr{F}(v(\mathcal{M})) \circ \omega(\mathscr{F}(\mathcal{M}))=\operatorname{id}_{\mathscr{F}(\mathcal{M})} .
$$

This proves (3.29) for any $\mathcal{M} \in \operatorname{Ob}\left(\mathscr{M}(\psi)_{\mathcal{D}}^{C}\right)$.

Next, we verify the condition (3.30). From the definition, it is clear that $\mathscr{G}$ preserves colimits. Since any $\mathcal{D}$-module may be expressed as the colimit of representable functors, it is enough to verify the condition (3.30) for representable functors. For any

$$
f \otimes d \in \mathbf{h}_{Y}(X) \otimes C,
$$


we have

$$
\begin{aligned}
\left(v\left(\mathscr{G}\left(\mathbf{h}_{Y}\right)\right)(X) \circ\right. & \left.\mathscr{G}\left(\omega\left(\mathbf{h}_{Y}\right)\right)(X)\right)(f \otimes d) \\
& =\left(v\left(\mathscr{G}\left(\mathbf{h}_{Y}\right)\right)(X) \circ\left(\omega\left(\mathbf{h}_{Y}\right) \otimes \mathrm{id}_{C}\right)(X)\right)(f \otimes d) \\
& =\left(v\left(\mathbf{h}_{Y} \otimes C\right)(X) \circ\left(\eta(X, Y) \otimes \mathrm{id}_{C}\right)\right)(f \otimes d) \\
& =\sum v\left(\mathbf{h}_{Y} \otimes C\right)(X)\left(\hat{f} \otimes c_{f} \otimes d\right) \\
& =\sum\left(\mathbf{h}_{Y} \otimes C\right)\left(\theta_{X}\left(\left(\hat{f} \otimes c_{f}\right)_{1} \otimes d\right)\right)\left(\hat{f} \otimes c_{f}\right)_{0} \\
& =\sum\left(\mathbf{h}_{Y} \otimes C\right)\left(\theta_{X}\left(c_{f_{2}} \otimes d\right)\right)\left(\hat{f} \otimes c_{f_{1}}\right) \\
& =\sum \mathbf{h}_{Y}\left(\left(\theta_{X}\left(c_{f_{2}} \otimes d\right)\right)_{\psi}\right)(\hat{f}) \otimes c_{f_{1}}{ }^{\psi} \\
& =\sum \hat{f} \circ\left(\theta_{X}\left(c_{f_{2}} \otimes d\right)\right)_{\psi} \otimes c_{f_{1}}{ } \\
& =\sum \hat{f} \circ\left(\theta_{X}\left(c_{f} \otimes d_{1}\right)\right) \otimes d_{2} \\
& =\varepsilon_{C}\left(d_{1}\right) f \otimes d_{2} \\
& =f \otimes d .
\end{aligned}
$$

This proves (3.30). Therefore, $(\mathscr{F}, \mathscr{G})$ is a Frobenius pair.

Conversely, suppose $(\mathscr{F}, \mathscr{G})$ is a Frobenius pair. Then, there exist $v \in V$ and $\omega \in W$ satisfying (3.29) and (3.30). Then, using the isomorphisms $V \cong V_{1}$ and $W \cong W_{1}$ as in Propositions 3.7 and 3.10, there exist $\theta \in V_{1}$ and $\eta \in W_{1}$ corrresponding to $v \in V$ and $\omega \in W$ respectively. We will now verify the conditions (3.31) and (3.32). Taking

$$
\mathcal{M}=C \otimes \mathbf{h}_{Y}
$$

in (3.29), for any $d \in C$ and $f \in \operatorname{Hom}_{\mathcal{D}}(X, Y)$ we have

$$
\begin{aligned}
d \otimes f & =\left(v\left(C \otimes \mathbf{h}_{Y}\right)(X) \circ \omega\left(C \otimes \mathbf{h}_{Y}\right)(X)\right)(d \otimes f) \\
& =\left(v\left(C \otimes \mathbf{h}_{Y}\right)(X) \circ\left(\operatorname{id}_{C} \otimes \omega_{\mathbf{h}_{Y}}(X)\right)\right)(d \otimes f) \\
& =v\left(C \otimes \mathbf{h}_{Y}\right)(X)(d \otimes \eta(X, Y)(f)) \\
& =\sum v\left(C \otimes \mathbf{h}_{Y}\right)(X)\left(d \otimes \hat{f} \otimes c_{f}\right) \\
& =\sum\left(C \otimes \mathbf{h}_{Y}\right)\left(\theta_{X}\left((d \otimes \hat{f})_{1} \otimes c_{f}\right)\right)(d \otimes \hat{f})_{0} \\
& =\sum\left(C \otimes \mathbf{h}_{Y}\right)\left(\theta_{X}\left(d_{2}^{\psi} \otimes c_{f}\right)\right)\left(d_{1} \otimes \hat{f}_{\psi}\right) \\
& =\sum d_{1} \otimes \hat{f}_{\psi} \circ \theta_{X}\left(d_{2}^{\psi} \otimes c_{f}\right)
\end{aligned}
$$


Applying $\varepsilon_{C} \otimes \operatorname{id}_{\mathbf{h}_{Y}(X)}$ on both sides, we get

$$
\begin{aligned}
\varepsilon_{C}(d) f & =\sum \varepsilon_{C}\left(d_{1}\right) \hat{f}_{\psi} \circ \theta_{X}\left(d_{2}{ }^{\psi} \otimes c_{f}\right) \\
& =\sum \varepsilon_{C}\left(d_{1}{ }^{\psi}\right) \hat{f}_{\psi_{\psi}} \circ \theta_{X}\left(d_{2}{ }^{\psi} \otimes c_{f}\right) \\
& =\sum \varepsilon_{C}\left(\left(d^{\psi}\right)_{1}\right) \hat{f}_{\psi} \circ \theta_{X}\left(\left(d^{\psi}\right)_{2} \otimes c_{f}\right) \\
& \left.=\sum \hat{f}_{\psi} \circ \theta_{X}\left(d^{\psi} \otimes c_{f}\right)\right) .
\end{aligned}
$$

This proves (3.32). Now, taking $\mathcal{N}=\mathbf{h}_{Y}$ in (3.30), we have

$$
\begin{aligned}
f \otimes d & =\left(v\left(\mathscr{G}\left(\mathbf{h}_{Y}\right)\right)(X) \circ \mathscr{G}\left(\omega\left(\mathbf{h}_{Y}\right)\right)(X)\right)(f \otimes d) \\
& =\left(v\left(\mathbf{h}_{Y} \otimes C\right)(X) \circ\left(\omega\left(\mathbf{h}_{Y}\right) \otimes \operatorname{id}_{C}\right)(X)\right)(f \otimes d) \\
& =\left(v\left(\mathbf{h}_{Y} \otimes C\right)(X) \circ\left(\eta(X, Y) \otimes \mathrm{id}_{C}\right)\right)(f \otimes d) \\
& =\sum v\left(\mathbf{h}_{Y} \otimes C\right)(X)\left(\hat{f} \otimes c_{f} \otimes d\right) \\
& =\sum\left(\mathbf{h}_{Y} \otimes C\right)\left(\theta_{X}\left(\left(\hat{f} \otimes c_{f}\right)_{1} \otimes d\right)\right)\left(\hat{f} \otimes c_{f}\right)_{0} \\
& =\sum\left(\mathbf{h}_{Y} \otimes C\right)\left(\theta_{X}\left(c_{f_{2}} \otimes d\right)\right)\left(\hat{f} \otimes c_{f_{1}}\right) \\
& =\sum \mathbf{h}_{Y}\left(\left(\theta_{X}\left(c_{f_{2}} \otimes d\right)\right)_{\psi}\right)(\hat{f}) \otimes c_{f_{1}}{ }^{\psi} \\
& =\sum \hat{f} \circ\left(\theta_{X}\left(c_{f_{2}} \otimes d\right)\right)_{\psi} \otimes c_{f_{1}}{ }^{\psi} \\
& =\sum \hat{f} \circ \theta_{X}\left(c_{f} \otimes d_{1}\right) \otimes d_{2}
\end{aligned}
$$

Applying

$$
\operatorname{id}_{\mathbf{h}_{Y}(X)} \otimes \varepsilon_{C}
$$

on both sides, we get (3.31). This proves the result.

3.3. Frobenius conditions in the CASe of a finite-dimensional CoALGEBRA. We continue with $(\mathcal{D}, C, \psi)$ being an entwining structure. For each $Y \in \operatorname{Ob}(\mathcal{D})$, we obtain an object $\operatorname{Hom}\left(C, \mathbf{h}_{Y}\right)$ in $\operatorname{Mod}-\mathcal{D}$ by setting

$$
\operatorname{Hom}\left(C, \mathbf{h}_{Y}\right)(X):=\operatorname{Hom}_{K}\left(C, \mathbf{h}_{Y}(X)\right)
$$

(3.36) $\operatorname{Hom}\left(C, \mathbf{h}_{Y}\right)(g): \operatorname{Hom}_{K}\left(C, \mathbf{h}_{Y}(X)\right) \longrightarrow \operatorname{Hom}_{K}\left(C, \mathbf{h}_{Y}\left(X^{\prime}\right)\right)$ given by

$$
\operatorname{Hom}\left(C, \mathbf{h}_{Y}\right)(g)(\phi)(x)=(\phi \cdot g)(x):=\phi(x) g,
$$

for any $X \in \operatorname{Ob}(\mathcal{D}), g \in \operatorname{Hom}_{\mathcal{D}}\left(X^{\prime}, X\right), \phi \in \operatorname{Hom}_{K}\left(C, \mathbf{h}_{Y}(X)\right)$ and $x \in C$. 
Using (3.36), we now define a functor $\operatorname{Hom}(C, h): \mathcal{D} \longrightarrow \operatorname{Mod}-\mathcal{D}$ as follows:

$$
\operatorname{Hom}(C, h)(Y):=\operatorname{Hom}\left(C, \mathbf{h}_{Y}\right),
$$

$$
\begin{gathered}
(\operatorname{Hom}(C, h)(f))(Z):\left(\operatorname{Hom}\left(C, \mathbf{h}_{Y}\right)\right)(Z) \longrightarrow\left(\operatorname{Hom}\left(C, \mathbf{h}_{X}\right)\right)(Z) \text { given by } \\
(\operatorname{Hom}(C, h)(f))(Z)(\phi)(x)=(f \cdot \phi)(x):=f_{\psi} \circ \phi\left(x^{\psi}\right),
\end{gathered}
$$

for any $f \in \operatorname{Hom}_{\mathcal{D}}(Y, X), \phi \in \operatorname{Hom}_{K}\left(C, \mathbf{h}_{Y}(Z)\right)$ and $x \in C$.

For the rest of this section, we assume that $C$ is finite-dimensional. Then, for each $Z \in \mathrm{Ob}(\mathcal{D})$, we have an isomorphism

$$
\operatorname{Hom}_{K}\left(C, \mathbf{h}_{Y}(Z)\right) \cong C^{*} \otimes \mathbf{h}_{Y}(Z) .
$$

Let $\left\{d_{i}\right\}_{1 \leq i \leq k}$ be a basis for $C$ and $\left\{d_{i}^{*}\right\}_{1 \leq i \leq k}$ be its dual basis.

Lemma 3.15: Let $C$ be a finite-dimensional coalgebra. Then, we have a functor

$$
C^{*} \otimes h: \mathcal{D} \longrightarrow \operatorname{Mod}-\mathcal{D}, \quad Y \mapsto C^{*} \otimes \mathbf{h}_{Y} .
$$

Proof. For each $Y \in \operatorname{Ob}(\mathcal{D})$, it is clear that $C^{*} \otimes \mathbf{h}_{Y} \in \operatorname{Mod}-\mathcal{D}$. We consider $f \in \operatorname{Hom}_{\mathcal{D}}(Y, X)$ and an element $c^{*} \otimes g \in C^{*} \otimes \mathbf{h}_{Y}(Z)$. By the isomorphism in (3.38), $c^{*} \otimes g$ corresponds to the element $\phi_{c^{*} \otimes g} \in \operatorname{Hom}_{K}\left(C, \mathbf{h}_{Y}(Z)\right)$ given by $\phi_{c^{*} \otimes g}(x)=c^{*}(x) g$ for each $x \in C$. From the action in (3.37), the element $f \cdot \phi_{c^{*} \otimes g} \in\left(\operatorname{Hom}\left(C, \mathbf{h}_{X}\right)\right)(Z)$ is given by

$$
\left(f \cdot \phi_{c^{*} \otimes g}\right)(x)=f_{\psi} \circ \phi_{c^{*} \otimes g}\left(x^{\psi}\right)=c^{*}\left(x^{\psi}\right)\left(f_{\psi} \circ g\right) .
$$

Again, using the isomorphism in (3.38), the element in $C^{*} \otimes \mathbf{h}_{X}(Z)$ corresponding to $f \cdot \phi_{c^{*} \otimes g}$ is given by

$$
\sum_{i=1}^{k} c^{*}\left(d_{i}^{\psi}\right) d_{i}^{*} \otimes f_{\psi} g .
$$

It may be easily verified that

$$
\left(C^{*} \otimes h\right)(f): C^{*} \otimes \mathbf{h}_{Y} \longrightarrow C^{*} \otimes \mathbf{h}_{X}
$$

is a morphism of right $\mathcal{D}$-modules. The result now follows.

Since $C$ is a coalgebra, its vector space dual $C^{*}$ is an algebra with the convolution product

$$
\left(c^{*} \cdot d^{*}\right)(x):=\sum c^{*}\left(x_{1}\right) d^{*}\left(x_{2}\right)
$$

for $c^{*}, d^{*} \in C^{*}$ and $x \in C$. Let $N$ be any left $C^{*}$-module. Then, we have a $K$-linear map $\rho: N \longrightarrow \operatorname{Hom}\left(C^{*}, N\right)$ defined by $\rho(n)\left(c^{*}\right):=c^{*} n$ for $n \in N$ and $c^{*} \in C^{*}$. 
In general, there is an embedding $N \otimes C \hookrightarrow \operatorname{Hom}\left(C^{*}, N\right)$ given by

$$
(n \otimes x)\left(c^{*}\right):=c^{*}(x) n
$$

for $x \in C$. Since $C$ is finite-dimensional, this embedding is also a surjection. This gives us a $K$-linear map $\rho: N \longrightarrow N \otimes C$ which makes $N$ a right $C$ comodule (see, for instance, $[17, \S 2.2]$ ). Then,

$$
\rho(n)=\sum_{i=1}^{k} d_{i}^{*} n \otimes d_{i} .
$$

In particular, $C^{*}$ becomes a right $C$-comodule with

$$
\rho_{C^{*}}\left(c^{*}\right)=\sum_{i=1}^{k} d_{i}^{*} \cdot c^{*} \otimes d_{i}
$$

Considering the element $\varepsilon_{C} \in C^{*}$, the coassociativity of the coaction $\rho_{C^{*}}$ may be used to verify that

$$
\sum_{j=1}^{k} \sum_{i=1}^{k}\left(d_{i}^{*} \cdot d_{j}^{*}\right) \otimes d_{i} \otimes d_{j}=\sum_{j=1}^{k} d_{j}^{*} \otimes \Delta\left(d_{j}\right) .
$$

Proposition 3.16: Let $C$ be a finite-dimensional coalgebra. Then, we have a functor

$$
\begin{aligned}
C^{*} \otimes h: \mathcal{D} \longrightarrow \mathscr{M}(\psi)_{\mathcal{D}}^{C} \\
Y \mapsto C^{*} \otimes \mathbf{h}_{Y} .
\end{aligned}
$$

Proof. From (3.40), we know that $C^{*}$ is a right $C$-comodule. Applying Lemma 2.5, it follows that each $C^{*} \otimes \mathbf{h}_{Y}$ is an object in $\mathscr{M}(\psi)_{\mathcal{D}}^{C}$. Accordingly, the right $C$-comodule structure on $C^{*} \otimes \mathbf{h}_{Y}(Z)$ for any $Z \in \mathrm{Ob}(\mathcal{D})$ is given by the following composition:

$$
\begin{array}{r}
\sigma_{C^{*} \otimes \mathbf{h}_{Y}(Z)}^{r}: C^{*} \otimes \mathbf{h}_{Y}(Z) \stackrel{\rho_{C^{*}} \otimes \mathrm{id}}{\longrightarrow} C^{*} \otimes C \otimes \mathbf{h}_{Y}(Z) \\
\stackrel{\operatorname{id} \otimes \psi_{Z Y}}{\longrightarrow} C^{*} \otimes \mathbf{h}_{Y}(Z) \otimes C .
\end{array}
$$

Explicitly, we have

$$
\sigma_{C^{*} \otimes \mathbf{h}_{Y}(Z)}^{r}\left(c^{*} \otimes g\right)=\sum_{i=1}^{k} d_{i}^{*} \cdot c^{*} \otimes g_{\psi} \otimes d_{i}^{\psi}
$$


for each $c^{*} \otimes g \in C^{*} \otimes \mathbf{h}_{Y}(Z)$. We consider $f \in \operatorname{Hom}_{\mathcal{D}}(Y, X)$. By Lemma 3.15, this induces a morphism $C^{*} \otimes \mathbf{h}_{Y} \longrightarrow C^{*} \otimes \mathbf{h}_{X}$ in Mod-D. In order to show that $C^{*} \otimes h: \mathcal{D} \longrightarrow \mathscr{M}(\psi)_{\mathcal{D}}^{C}$ is a functor, it therefore suffices to show that each morphism

$$
C^{*} \otimes \mathbf{h}_{Y}(Z) \longrightarrow C^{*} \otimes \mathbf{h}_{X}(Z),
$$

$$
\left(c^{*} \otimes g\right) \mapsto \sum_{j=1}^{k} c^{*}\left(d_{j}^{\psi}\right) d_{j}^{*} \otimes f_{\psi} g,
$$

is right $C$-colinear. For any $c^{*} \otimes g \in C^{*} \otimes \mathbf{h}_{Y}(Z)$, we have

$$
\begin{aligned}
& \sigma_{C^{*} \otimes \mathbf{h}_{X}(Z)}^{r}\left(f \cdot\left(c^{*} \otimes g\right)\right) \\
&=\sum_{j=1}^{k} \sigma_{C^{*} \otimes \mathbf{h}_{X}(Z)}^{r}\left(c^{*}\left(d_{j}^{\psi}\right) d_{j}^{*} \otimes f_{\psi} g\right) \\
&=\sum_{i=1}^{k} \sum_{j=1}^{k} c^{*}\left(d_{j}^{\psi}\right) d_{i}^{*} \bullet d_{j}^{*} \otimes\left(f_{\psi} g\right)_{\psi} \otimes d_{i}^{\psi} \\
&=\sum_{i=1}^{k} \sum_{j=1}^{k} c^{*}\left(d_{j}^{\psi}\right) d_{i}^{*} \bullet d_{j}^{*} \otimes f_{\psi_{\psi}} g_{\psi} \otimes d_{i}^{\psi} \psi \\
&=\sum_{j=1}^{k} c^{*}\left(d_{j_{2}}{ }^{\psi}\right) d_{j}^{*} \otimes f_{\psi_{\psi}} g_{\psi} \otimes d_{j_{1}} \psi^{\psi} \\
&=\sum_{j=1}^{k} c^{*}\left(d_{j_{2}}{ }^{\psi}\right) d_{j}^{*} \otimes f_{\psi_{\psi}} g_{\psi} \otimes\left(\sum_{i=1}^{k} d_{i}^{*}\left(d_{j_{1}}{ }^{\psi}\right) d_{i}^{\psi}\right) \\
&=\sum_{j=1}^{k} \sum_{i=1}^{k} d_{i}^{*}\left(d_{j_{1}}{ }^{\psi}\right) c^{*}\left(d_{j_{2}}{ }^{\psi}\right) d_{j}^{*} \otimes f_{\psi_{\psi}} g_{\psi} \otimes d_{i}^{\psi} \\
&=\sum_{j=1}^{k} \sum_{i=1}^{k} d_{i}^{*}\left(\left(d_{j}{ }^{\psi}\right)_{1}\right) c^{*}\left(\left(d_{j}{ }^{\psi}\right)_{2}\right) d_{j}^{*} \otimes f_{\psi} g_{\psi} \otimes d_{i}^{\psi} \\
&= \sum_{j=1}^{k} \sum_{i=1}^{k}\left(d_{i}^{*} \bullet c^{*}\right)\left(d_{j}^{\psi}\right) d_{j}^{*} \otimes f_{\psi} g_{\psi} \otimes d_{i}^{\psi} \\
&=\left(f \otimes \operatorname{id}_{C}\right) \cdot\left(\sum_{i=1}^{k}\left(d_{i}^{*} \bullet c^{*}\right) \otimes g_{\psi} \otimes d_{i}^{\psi}\right) \\
&=\left(f \otimes \operatorname{id}_{C}\right) \cdot\left(\sigma_{C^{*}}^{r} \otimes \mathbf{h}_{Y}(Z)\right. \\
&\left.\left(c^{*} \otimes g\right)\right) . \\
& \mathbf{\square}
\end{aligned}
$$


Since $C$ is finite-dimensional, the right $C$-comodule structure on $C^{*} \otimes \mathbf{h}_{Y}(X)$ induces a right $C$-comodule structure on $\operatorname{Hom}\left(C, \mathbf{h}_{Y}(X)\right)$ for each $X, Y \in \operatorname{Ob}(\mathcal{D})$ which we now explain. Let $\phi \in \operatorname{Hom}\left(C, \mathbf{h}_{Y}(X)\right)$. Then, $\phi$ corresponds to the element

$$
\sum_{1 \leq i \leq k} d_{i}^{*} \otimes \phi\left(d_{i}\right) \in C^{*} \otimes \mathbf{h}_{Y}(X)
$$

We know by Proposition 3.16 that

$$
\sigma_{C^{*} \otimes \mathbf{h}_{Y}(X)}^{r}\left(\sum_{i=1}^{k} d_{i}^{*} \otimes \phi\left(d_{i}\right)\right)=\sum_{j=1}^{k} \sum_{i=1}^{k} d_{j}^{*} \bullet d_{i}^{*} \otimes\left(\phi\left(d_{i}\right)\right)_{\psi} \otimes d_{j}^{\psi} .
$$

The element $\sum_{i=1}^{k} d_{j}^{*} \bullet d_{i}^{*} \otimes\left(\phi\left(d_{i}\right)\right)_{\psi} \otimes d_{j}^{\psi} \in C^{*} \otimes \mathbf{h}_{Y}(X) \otimes C$ corresponds to the element $\phi_{0} \otimes \phi_{1} \in \operatorname{Hom}\left(C, \mathbf{h}_{Y}(X)\right) \otimes C$ given by

$$
\begin{aligned}
\phi_{0}(x) \otimes \phi_{1} & =\sum_{j=1}^{k} \sum_{i=1}^{k}\left(d_{j}^{*} \bullet d_{i}^{*}\right)(x)\left(\phi\left(d_{i}\right)\right)_{\psi} \otimes d_{j}^{\psi} \\
& =\sum_{j=1}^{k} \sum_{i=1}^{k} d_{j}^{*}\left(x_{1}\right) d_{i}^{*}\left(x_{2}\right)\left(\phi\left(d_{i}\right)\right)_{\psi} \otimes d_{j}^{\psi} \\
& =\psi\left(x_{1} \otimes \phi\left(x_{2}\right)\right)
\end{aligned}
$$

for $x \in C$. It now follows from (3.36), (3.37), (3.43) and Proposition 3.16 that we have a functor

$$
\operatorname{Hom}(C, h): \mathcal{D} \longrightarrow \mathscr{M}(\psi)_{\mathcal{D}}^{C}, \quad Y \mapsto \operatorname{Hom}_{K}\left(C, \mathbf{h}_{Y}\right) .
$$

We also recall from (3.3) and (3.4), the functor $h \otimes C: \mathcal{D} \longrightarrow \mathscr{M}(\psi)_{\mathcal{D}}^{C}$, defined as follows:

$$
\begin{aligned}
& (h \otimes C)(Y):=\mathbf{h}_{Y} \otimes C, \\
& (h \otimes C)(f)(Z)(g \otimes c):=f g \otimes c,
\end{aligned}
$$

for $f \in \operatorname{Hom}_{\mathcal{D}}(Y, X)$ and $g \otimes c \in \mathbf{h}_{Y}(Z) \otimes C$. We now set

$$
V_{2}:=\operatorname{Nat}\left(h \otimes C, C^{*} \otimes h\right) .
$$

Proposition 3.17: Let $C$ be a finite-dimensional coalgebra. Then,

$$
V=\operatorname{Nat}\left(\mathscr{G} \mathscr{F}, 1_{\mathscr{M}(\psi)_{\mathcal{D}}^{C}}\right) \cong V_{1} \cong V_{2}=\operatorname{Nat}\left(h \otimes C, C^{*} \otimes h\right) .
$$

Proof. Since $C$ is finite-dimensional, we know that

$$
C^{*} \otimes \mathbf{h}_{Y}(X) \cong \operatorname{Hom}_{K}\left(C, \mathbf{h}_{Y}(X)\right) \text { for each } X, Y \in \operatorname{Ob}(\mathcal{D}) .
$$


We first define a $K$-linear map $\Upsilon_{X Y}: \mathbf{h}_{Y}(X) \otimes C \longrightarrow C^{*} \otimes \mathbf{h}_{Y}(X)$ given by

$$
\left(\Upsilon_{X Y}(f \otimes c)\right)(d):=f_{\psi} \circ \theta_{X}\left(d^{\psi} \otimes c\right)
$$

for any $f \in \operatorname{Hom}_{\mathcal{D}}(X, Y)$ and $c, d \in C$. In other words, we have

$$
\Upsilon_{X Y}(f \otimes c)=\sum_{i=1}^{k} d_{i}^{*} \otimes\left(f_{\psi} \circ \theta_{X}\left(d_{i}^{\psi} \otimes c\right)\right)
$$

where $\left\{d_{i}\right\}_{1 \leq i \leq k}$ is a basis for $C$ and $\left\{d_{i}^{*}\right\}_{1 \leq i \leq k}$ is its dual basis.

We now define $\alpha^{\prime}: V_{1} \longrightarrow V_{2}$ by setting $\alpha^{\prime}(\theta)=\Upsilon$ with $\Upsilon: h \otimes C \longrightarrow C^{*} \otimes h$ defined as follows:

$$
\Upsilon_{Y}: \mathbf{h}_{Y} \otimes C \longrightarrow C^{*} \otimes \mathbf{h}_{Y}, \quad \Upsilon_{Y}(X):=\Upsilon_{X Y},
$$

for any $X, Y \in \operatorname{Ob}(\mathcal{D})$. We now verify that $\alpha^{\prime}$ is a well-defined map. For this, we first check that $\Upsilon_{Y}: \mathbf{h}_{Y} \otimes C \longrightarrow C^{*} \otimes \mathbf{h}_{Y}$ is a morphism in $\mathscr{M}(\psi)_{\mathcal{D}}^{C}$ for every $Y \in \operatorname{Ob}(\mathcal{D})$. For any $g \in \operatorname{Hom}_{\mathcal{D}}\left(X^{\prime}, X\right)$, we need to show that the following diagram commutes:

$$
\begin{array}{ccc}
\mathbf{h}_{Y}(X) \otimes C \stackrel{\Upsilon_{Y}(X)}{\longrightarrow} C^{*} \otimes \mathbf{h}_{Y}(X) \\
\left(\mathbf{h}_{Y} \otimes C\right)(g) \downarrow & \downarrow\left(C^{*} \otimes \mathbf{h}_{Y}\right)(g) \\
\mathbf{h}_{Y}\left(X^{\prime}\right) \otimes C & \stackrel{\Upsilon_{Y}\left(X^{\prime}\right)}{\longrightarrow} C^{*} \otimes \mathbf{h}_{Y}\left(X^{\prime}\right)
\end{array}
$$

For any $f \otimes c \in \mathbf{h}_{Y}(X) \otimes C$, we have

$$
\begin{aligned}
\left(C^{*} \otimes \mathbf{h}_{Y}\right) & (g) \Upsilon_{Y}(X)(f \otimes c) \\
& =\sum_{i=1}^{k}\left(C^{*} \otimes \mathbf{h}_{Y}\right)(g)\left(d_{i}^{*} \otimes f_{\psi} \circ \theta_{X}\left(d_{i}^{\psi} \otimes c\right)\right) \\
& =\sum_{i=1}^{k} d_{i}^{*} \otimes\left(f_{\psi} \circ \theta_{X}\left(d_{i}^{\psi} \otimes c\right)\right) \circ g \\
& =\sum_{i=1}^{k} d_{i}^{*} \otimes f_{\psi} g_{\psi} \circ \theta_{X^{\prime}}\left(d_{i}^{\psi} \psi \otimes c^{\psi}\right) \\
& =\sum_{i=1}^{k} d_{i}^{*} \otimes\left(f g_{\psi}\right)_{\psi} \circ \theta_{X^{\prime}}\left(d_{i}^{\psi} \otimes c^{\psi}\right) \\
& =\Upsilon_{Y}\left(X^{\prime}\right)\left(f g_{\psi} \otimes c^{\psi}\right)=\Upsilon_{Y}\left(X^{\prime}\right)\left(\mathbf{h}_{Y} \otimes C\right)(g)(f \otimes c) .
\end{aligned}
$$


This shows that $\Upsilon_{Y}$ is a morphism of right $\mathcal{D}$-modules for every $Y \in \operatorname{Ob}(\mathcal{D})$. Next we verify that

$$
\Upsilon_{Y}(X): \mathbf{h}_{Y}(X) \otimes C \longrightarrow C^{*} \otimes \mathbf{h}_{Y}(X)
$$

is right $C$-colinear for every $X, Y \in \mathrm{Ob}(\mathcal{D})$. We have

$$
\begin{aligned}
\sigma_{C^{*} \otimes \mathbf{h}_{Y}(X)}^{r} & \left(\Upsilon_{Y}(X)(f \otimes c)\right) \\
& =\sum_{i=1}^{k} \sigma_{C^{*} \otimes \mathbf{h}_{Y}(X)}^{r}\left(d_{i}^{*} \otimes f_{\psi} \circ \theta_{X}\left(d_{i}^{\psi} \otimes c\right)\right) \\
& =\sum_{i, j=1}^{k} d_{j}^{*} \bullet d_{i}^{*} \otimes\left(f_{\psi} \circ \theta_{X}\left(d_{i}^{\psi} \otimes c\right)\right)_{\psi} \otimes d_{j}^{\psi} \\
& =\sum_{i=1}^{k} d_{i}^{*} \otimes\left(f_{\psi} \circ \theta_{X}\left(d_{i_{2}}{ }^{\psi} \otimes c\right)\right)_{\psi} \otimes d_{i_{1}}{ }^{\psi} \\
& =\sum_{i=1}^{k} d_{i}^{*} \otimes f_{\psi} \circ\left(\theta_{X}\left(d_{i_{2}}{ }^{\psi} \otimes c\right)\right)_{\psi} \otimes d_{i_{1}}{ }^{\psi} \\
& =\sum_{i=1}^{k} d_{i}^{*} \otimes f_{\psi} \circ\left(\theta_{X}\left(\left(d_{i}^{\psi}\right)_{2} \otimes c\right)\right)_{\psi} \otimes\left(d_{i}^{\psi}\right)_{1}{ }^{\psi} \\
& =\sum_{i=1}^{k} d_{i}^{*} \otimes f_{\psi} \circ \theta_{X}\left(d_{i}^{\psi} \otimes c_{1}\right) \otimes c_{2} \\
& =\Upsilon_{Y}(X)\left(f \otimes c_{1}\right) \otimes c_{2} \\
& =\left(\Upsilon_{Y}(X) \otimes \operatorname{id}_{C}\right)\left(\pi_{\mathbf{h}_{Y}(X) \otimes C}^{r}(f \otimes c)\right) .
\end{aligned}
$$

Finally, we verify that $\Upsilon$ is a natural transformation from $h \otimes C$ to $C^{*} \otimes h$, i.e., the following diagram commutes for any $g \in \operatorname{Hom}_{\mathcal{D}}\left(Y, Y^{\prime}\right)$ :

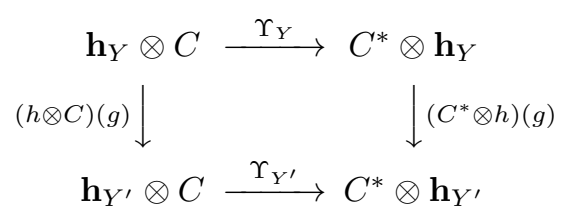


For any $f \otimes c \in \mathbf{h}_{Y}(X) \otimes C$, we have

$$
\begin{aligned}
\left(C^{*} \otimes h\right)(g)(X) \Upsilon_{Y}(X)(f \otimes c) \\
=\sum_{i=1}^{k}\left(C^{*} \otimes h\right)(g)(X)\left(d_{i}^{*} \otimes f_{\psi} \circ \theta_{X}\left(d_{i}^{\psi} \otimes c\right)\right) \\
=\sum_{i, j=1}^{k} d_{i}^{*}\left(d_{j}^{\psi}\right) d_{j}^{*} \otimes g_{\psi} f_{\psi} \theta_{X}\left(d_{i}^{\psi} \otimes c\right) \\
=\sum_{j=1}^{k} d_{j}^{*} \otimes g_{\psi} f_{\psi} \circ \theta_{X}\left(\sum_{i=1}^{n} d_{i}^{*}\left(d_{j}{ }^{\psi}\right) d_{i}{ }^{\psi} \otimes c\right) \\
=\sum_{j=1}^{k} d_{j}^{*} \otimes g_{\psi} f_{\psi} \circ \theta_{X}\left(d_{j}^{\psi^{\psi}} \otimes c\right) \\
=\sum_{j=1}^{k} d_{j}^{*} \otimes(g f)_{\psi} \circ \theta_{X}\left(d_{j}^{\psi} \otimes c\right) \\
=\Upsilon_{Y^{\prime}}(X)(g f \otimes c)=\Upsilon_{Y^{\prime}}(X)(h \otimes C)(g)(X)(f \otimes c) .
\end{aligned}
$$

This proves that $\Upsilon \in V_{2}$.

For the converse, we first observe that the functors $C^{*} \otimes h$ and $\operatorname{Hom}(C, h)$ are isomorphic which follows from (3.38). We define $\beta^{\prime}: V_{2} \longrightarrow V_{1}$ by setting

$$
\beta^{\prime}(\Upsilon)=\theta
$$

with $\theta_{X}: C \otimes C \longrightarrow \operatorname{End}_{\mathcal{D}}(X)$ defined as follows:

$$
\theta_{X}(c \otimes d):=\left(\Upsilon_{X X}\left(\operatorname{id}_{X} \otimes d\right)\right)(c)
$$

for any $X \in \mathrm{Ob}(\mathcal{D})$ and $c, d \in C$. We will now verify that $\theta$ satisfies $(3.11)$ and (3.12). For each $X \in \mathrm{Ob}(\mathcal{D})$, we know that $\Upsilon_{X}: \mathbf{h}_{X} \otimes C \longrightarrow \operatorname{Hom}\left(C, \mathbf{h}_{X}\right)$ is a morphism of right $\mathcal{D}$-modules. Therefore, for any $f \in \operatorname{Hom}_{\mathcal{D}}(Y, X)$, we have the following commutative diagram:

$$
\begin{array}{cc}
\mathbf{h}_{X}(X) \otimes C \stackrel{\Upsilon_{X}(X)}{\longrightarrow} \operatorname{Hom}_{K}\left(C, \mathbf{h}_{X}(X)\right) \\
\left(\mathbf{h}_{X} \otimes C\right)(f) \downarrow & \downarrow \operatorname{Hom}\left(C, \mathbf{h}_{X}\right)(f) \\
\mathbf{h}_{X}(Y) \otimes C & \stackrel{\Upsilon_{X}(Y)}{\longrightarrow} \operatorname{Hom}_{K}\left(C, \mathbf{h}_{X}(Y)\right) .
\end{array}
$$


Since $\Upsilon: h \otimes C \longrightarrow \operatorname{Hom}(C, h)$ is a natural transformation, the following diagram also commutes for any $f \in \operatorname{Hom}_{D}(Y, X)$ :

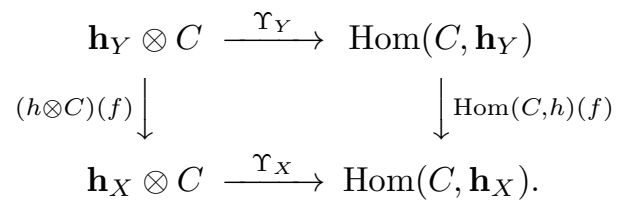

Therefore, we have

$$
\begin{aligned}
\theta_{X}(c \otimes d) \circ f & =\left(\left(\Upsilon_{X X}\left(\operatorname{id}_{X} \otimes d\right)\right)(c)\right) \circ f \\
& =\left(\left(\Upsilon_{X X}\left(\operatorname{id}_{X} \otimes d\right)\right) \cdot f\right)(c) \\
& =\left(\Upsilon_{X}(Y)\left(h_{X} \otimes C\right)(f)\left(\operatorname{id}_{X} \otimes d\right)\right)(c) \\
& =\left(\Upsilon_{X}(Y) h_{X}\left(f_{\psi}\right)\left(\operatorname{id}_{X}\right) \otimes d^{\psi}\right)(c) \\
& =\Upsilon_{Y X}\left(f_{\psi} \otimes d^{\psi}\right)(c) \\
& =\Upsilon_{Y X} \circ\left((h \otimes C)\left(f_{\psi}\right)(Y)\left(\operatorname{id}_{Y} \otimes d^{\psi}\right)\right)(c) \\
& =\left(f_{\psi} \cdot \Upsilon_{Y Y}\left(\operatorname{id}_{Y} \otimes d^{\psi}\right)\right)(c) \\
& =f_{\psi_{\psi}} \circ\left(\Upsilon_{Y Y}\left(\operatorname{id}_{Y} \otimes d^{\psi}\right)\right)\left(c^{\psi}\right) \\
& =f_{\psi_{\psi}} \circ\left(\theta_{Y}\left(c^{\psi} \otimes d^{\psi}\right)\right) .
\end{aligned}
$$

This proves (3.11). Further, we have

$$
\begin{array}{rlrl}
\left(\theta _ { X } \left(c_{2}\right.\right. & \otimes d))_{\psi} \otimes c_{1} \psi \\
& =\psi\left(c_{1} \otimes \theta_{X}\left(c_{2} \otimes d\right)\right) & \\
& =\psi\left(c_{1} \otimes\left(\Upsilon_{X X}\left(\operatorname{id}_{X} \otimes d\right)\right)\left(c_{2}\right)\right) & \\
& =\left(\Upsilon_{X X}\left(\operatorname{id}_{X} \otimes d\right)\right)_{0}(c) \otimes\left(\Upsilon_{X X}\left(\operatorname{id}_{X} \otimes d\right)\right)_{1} & & (\text { by }(3.43)) \\
& =\left(\Upsilon_{X X}\left(\operatorname{id}_{X} \otimes d\right)_{0}\right)(c) \otimes\left(\operatorname{id}_{X} \otimes d\right)_{1} & & \left(\Upsilon_{X X} \text { is } C \text {-colinear }\right) \\
& =\left(\Upsilon_{X X}\left(\operatorname{id}_{X} \otimes d_{1}\right)\right)(c) \otimes d_{2} & & (\text { by }(2.6)) \\
& =\theta_{X}\left(c \otimes d_{1}\right) \otimes d_{2} . &
\end{array}
$$

This proves (3.12). It remains to show that $\alpha^{\prime}$ and $\beta^{\prime}$ are inverses of each other. For every $\theta \in V_{1}$ and $c, d \in C$, it follows from (3.45) that

$$
\left(\left(\beta^{\prime} \circ \alpha^{\prime}\right)(\theta)\right)_{X}(c \otimes d)=\left(\alpha^{\prime}(\theta)\right)_{X X}\left(\operatorname{id}_{X} \otimes d\right)(c)=\theta_{X}(c \otimes d) .
$$


Finally, for any $\Upsilon \in V_{2}, f \in \operatorname{Hom}_{\mathcal{D}}(X, Y)$ and $c, d \in C$, we have

$$
\begin{aligned}
\left(\left(\alpha^{\prime} \circ \beta^{\prime}\right)(\Upsilon)\right)_{X Y}(f \otimes c)(d) & =\sum_{i=1}^{k} d_{i}^{*}(d) f_{\psi} \circ\left(\left(\beta^{\prime}(\Upsilon)\right)_{X}\left(d_{i}^{\psi} \otimes c\right)\right) \\
& =\sum_{i=1}^{k} d_{i}^{*}(d) f_{\psi} \circ\left(\Upsilon_{X X}\left(\operatorname{id}_{X} \otimes c\right)\left(d_{i}^{\psi}\right)\right) \\
& =f_{\psi} \circ\left(\Upsilon_{X X}\left(\operatorname{id}_{X} \otimes c\right)\left(d^{\psi}\right)\right) \\
& =\left(f \cdot\left(\Upsilon_{X X}\left(\operatorname{id}_{X} \otimes c\right)\right)\right)(d) \\
& =\left(\Upsilon_{X Y}(f \otimes c)\right)(d)
\end{aligned}
$$

This proves the result.

Proposition 3.18: Let $C$ be a finite-dimensional coalgebra. Then, we have isomorphisms

$$
W=\operatorname{Nat}\left(1_{\text {Mod }-\mathcal{D}}, \mathscr{F} \mathscr{G}\right) \cong W_{1}=\operatorname{Nat}(h, h \otimes C) \cong W_{2}:=\operatorname{Nat}\left(C^{*} \otimes h, h \otimes C\right) .
$$

Proof. Given an $\eta: h \longrightarrow h \otimes C$, we want to define $\Phi: C^{*} \otimes h \longrightarrow h \otimes C$. For each $Y \in \mathrm{Ob}(\mathcal{D})$, we first define a $K$-linear map $\Phi_{Y Y}: C^{*} \otimes \mathbf{h}_{Y}(Y) \longrightarrow \mathbf{h}_{Y}(Y) \otimes C$ by the following composition:

$$
\begin{gathered}
C^{*} \otimes \mathbf{h}_{Y}(Y) \stackrel{\operatorname{id}_{C^{*}} \otimes \eta(Y, Y)}{\longrightarrow} C^{*} \otimes \mathbf{h}_{Y}(Y) \otimes C \stackrel{\mathrm{id}_{C^{*} \otimes \mathbf{h}_{Y}(Y)} \otimes \Delta_{C}}{\longrightarrow} C^{*} \otimes \mathbf{h}_{Y}(Y) \otimes C \otimes C \\
\stackrel{\tau \otimes \mathrm{id}_{C}}{\longrightarrow} \mathbf{h}_{Y}(Y) \otimes C \otimes\left(C^{*} \otimes C\right) \stackrel{\mathrm{ev}}{\longrightarrow} \mathbf{h}_{Y}(Y) \otimes C,
\end{gathered}
$$

i.e.,

$$
\Phi_{Y Y}\left(c^{*} \otimes \operatorname{id}_{Y}\right)=\sum a_{Y} \otimes c^{*}\left(c_{Y_{2}}\right) c_{Y_{1}}
$$

where

$$
\sum a_{Y} \otimes c_{Y}=\eta(Y, Y)\left(\operatorname{id}_{Y}\right)
$$

as in the notation of Lemma 3.9. We observe that an element $c^{*} \otimes f \in C^{*} \otimes \mathbf{h}_{Y}(X)$ may be written as

$$
c^{*} \otimes f=\left(C^{*} \otimes \mathbf{h}_{Y}\right)(f)\left(c^{*} \otimes \operatorname{id}_{Y}\right) .
$$

For each $X \in \mathrm{Ob}(\mathcal{D})$, we now define $\Phi_{X Y}: C^{*} \otimes \mathbf{h}_{Y}(X) \longrightarrow \mathbf{h}_{Y}(X) \otimes C$ as follows:

(3.49) $\Phi_{X Y}\left(c^{*} \otimes f\right):=\left(\mathbf{h}_{Y} \otimes C\right)(f)\left(\Phi_{Y Y}\left(c^{*} \otimes \operatorname{id}_{Y}\right)\right)=\sum a_{Y} f_{\psi} \otimes c^{*}\left(c_{Y_{2}}\right) c_{Y_{1}} \psi$ for any $c^{*} \otimes f \in C^{*} \otimes \mathbf{h}_{Y}(X)$. 
We define $\gamma^{\prime}: W_{1} \longrightarrow W_{2}$ by setting $\gamma^{\prime}(\eta)=\Phi$ with $\Phi: C^{*} \otimes h \longrightarrow h \otimes C$ given by

$$
\Phi_{Y}: C^{*} \otimes \mathbf{h}_{Y} \longrightarrow \mathbf{h}_{Y} \otimes C, \quad \Phi_{Y}(X):=\Phi_{X Y},
$$

for every $X, Y \in \operatorname{Ob}(\mathcal{D})$. We now verify that $\gamma^{\prime}$ is a well-defined map. For this, we first check that $\Phi_{Y}: C^{*} \otimes \mathbf{h}_{Y} \longrightarrow \mathbf{h}_{Y} \otimes C$ is a morphism of right $\mathcal{D}$-modules for every $Y \in \operatorname{Ob}(\mathcal{D})$, i.e., the following diagram commutes for any $g \in \operatorname{Hom}_{\mathcal{D}}\left(X^{\prime}, X\right)$ :

$$
\begin{array}{cc}
C^{*} \otimes \mathbf{h}_{Y}(X) \stackrel{\Phi_{Y}(X)}{\longrightarrow} & \mathbf{h}_{Y}(X) \otimes C \\
\left(C^{*} \otimes \mathbf{h}_{Y}\right)(g) \downarrow & \downarrow\left(\mathbf{h}_{Y} \otimes C\right)(g) \\
C^{*} \otimes \mathbf{h}_{Y}\left(X^{\prime}\right) \stackrel{\Phi_{Y}\left(X^{\prime}\right)}{\longrightarrow} & \mathbf{h}_{Y}\left(X^{\prime}\right) \otimes C
\end{array}
$$

We have

$$
\begin{aligned}
\Phi_{Y}\left(X^{\prime}\right)\left(C^{*} \otimes \mathbf{h}_{Y}\right)(g)\left(c^{*} \otimes f\right) & =\Phi_{Y}\left(X^{\prime}\right)\left(c^{*} \otimes f g\right)=\sum a_{Y}(f g)_{\psi} \otimes c^{*}\left(c_{Y_{2}}\right) c_{Y_{1}}{ }^{\psi} \\
& =\sum a_{Y} f_{\psi} g_{\psi} \otimes c^{*}\left(c_{Y_{2}}\right) c_{Y_{1}}{ }^{\psi} \\
& =\left(\mathbf{h}_{Y} \otimes C\right)(g)\left(\Phi_{Y}(X)\left(c^{*} \otimes f\right)\right) .
\end{aligned}
$$

Next we verify that $\Phi_{Y}(X): C^{*} \otimes \mathbf{h}_{Y}(X) \longrightarrow \mathbf{h}_{Y}(X) \otimes C$ is right $C$-colinear for any $X, Y \in \mathrm{Ob}(\mathcal{D})$ :

$$
\begin{aligned}
\left(\Phi_{Y}(X) \otimes \operatorname{id}_{C}\right) & \left(\sigma_{C^{*} \otimes \mathbf{h}_{Y}(X)}^{r}\left(c^{*} \otimes f\right)\right) \\
& =\sum_{i=1}^{k} \Phi_{Y}(X)\left(d_{i}^{*} \bullet c^{*} \otimes f_{\psi}\right) \otimes d_{i}^{\psi} \\
& =\sum_{i=1}^{k} \sum a_{Y} f_{\psi_{\psi}} \otimes\left(d_{i}^{*} \bullet c^{*}\right)\left(c_{Y_{2}}\right) c_{Y_{1}}{ }^{\psi} \otimes d_{i}^{\psi} \\
& =\sum_{i=1}^{k} \sum a_{Y} f_{\psi_{\psi}} \otimes d_{i}^{*}\left(c_{Y_{2}}\right) c^{*}\left(c_{Y_{3}}\right) c_{Y_{1}}{ }^{\psi} \otimes d_{i}^{\psi} \\
& =\sum a_{Y} f_{\psi_{\psi}} \otimes c^{*}\left(c_{Y_{3}}\right) c_{Y_{1}}{ }^{\psi} \otimes c_{Y_{2}}{ }^{\psi} \\
& =\sum a_{Y} f_{\psi_{\psi}} \otimes c^{*}\left(c_{Y_{2}}\right)\left(c_{Y_{1}}\right)_{1}{ }^{\psi} \otimes\left(c_{Y_{1}}\right)_{2}{ }^{\psi} \\
& =\sum a_{Y} f_{\psi} \otimes c^{*}\left(c_{Y_{2}}\right)\left(c_{Y_{1}}{ }^{\psi}\right)_{1} \otimes\left(c_{Y_{1}}{ }^{\psi}\right)_{2} \\
& =\pi_{\mathbf{h}_{Y}(X) \otimes C}^{r}\left(\Phi_{Y}(X)\left(c^{*} \otimes f\right)\right) .
\end{aligned}
$$


It follows that $\Phi_{Y}: C^{*} \otimes \mathbf{h}_{Y} \longrightarrow \mathbf{h}_{Y} \otimes C$ is a morphism in $\mathscr{M}(\psi)_{\mathcal{D}}^{C}$. To show that $\Phi \in \operatorname{Nat}\left(C^{*} \otimes h, h \otimes C\right)$, it remains to verify that the following diagram commutes:

$$
\begin{gathered}
C^{*} \otimes \mathbf{h}_{Y} \stackrel{\Phi_{Y}}{\longrightarrow} \mathbf{h}_{Y} \otimes C \\
\left(C^{*} \otimes h\right)(g) \downarrow \\
C^{*} \otimes \mathbf{h}_{Z} \stackrel{\Phi_{Z}}{\longrightarrow} \mathbf{h}_{Z} \otimes C
\end{gathered}
$$

for any $g \in \operatorname{Hom}_{\mathcal{D}}(Y, Z)$. For any $X \in \operatorname{Ob}(\mathcal{D})$ and $c^{*} \otimes f \in C^{*} \otimes \mathbf{h}_{Y}(X)$, we have

$$
\begin{aligned}
\Phi_{Z} & (X)\left(C^{*} \otimes h\right)(g)(X)\left(c^{*} \otimes f\right) \\
& =\sum_{i=1}^{k} \Phi_{Z}(X)\left(c^{*}\left(d_{i}^{\psi}\right) d_{i}^{*} \otimes g_{\psi} f\right) \\
& =\sum_{i=1}^{k} \sum c^{*}\left(d_{i}^{\psi}\right) a_{Z}\left(g_{\psi} f\right)_{\psi} \otimes d_{i}^{*}\left(c_{Z_{2}}\right) c_{Z_{1}}{ }^{\psi} \\
& =\sum_{i=1}^{k} \sum c^{*}\left(d_{i}^{\psi}\right) a_{Z} g_{\psi_{\psi}} f_{\psi} \otimes d_{i}^{*}\left(c_{Z_{2}}\right) c_{Z_{1}}{ }^{\psi}{ }^{\psi} \\
& =\sum c^{*}\left(c_{Z_{2}}{ }^{\psi}\right) a_{Z} g_{\psi} f_{\psi} \otimes c_{Z_{1}}{ }^{\psi}{ }^{\psi} \\
& =\sum c^{*}\left(\left(c_{Z}{ }^{\psi}\right)_{2}\right) a_{Z} g_{\psi} f_{\psi} \otimes\left(c_{Z}\right)_{1}{ }^{\psi} \\
& =\sum c^{*}\left(c_{Y_{2}}\right) g a_{Y} f_{\psi} \otimes\left(c_{Y_{1}}\right)^{\psi} \\
& =(h \otimes C)(g)(X) \Phi_{Y}(X)\left(c^{*} \otimes f\right) .
\end{aligned}
$$

Conversely, we define $\delta^{\prime}: W_{2} \longrightarrow W_{1}$ by setting $\delta^{\prime}(\Phi)=\eta$ with $\eta: h \longrightarrow h \otimes C$ given by

$$
\eta(X, Y)(f):=\Phi_{Y}(X)\left(\varepsilon_{C} \otimes f\right)
$$

for any $(X, Y) \in \operatorname{Ob}\left(\mathcal{D}^{o p} \otimes \mathcal{D}\right)$ and $f \in \operatorname{Hom}_{\mathcal{D}}(X, Y)$. We now verify that $\eta \in W_{1}$. Let $\phi:(X, Y) \longrightarrow\left(X^{\prime}, Y^{\prime}\right)$ be a morphism in $\mathcal{D}^{o p} \otimes \mathcal{D}$ given by $\phi^{\prime}: X^{\prime} \longrightarrow X$ and $\phi^{\prime \prime}: Y \longrightarrow Y^{\prime}$ in $\mathcal{D}$. Then, using the fact that $\Phi_{Y}: C^{*} \otimes \mathbf{h}_{Y} \longrightarrow \mathbf{h}_{Y} \otimes C$ is a morphism of right $\mathcal{D}$-modules, we have

$$
\begin{aligned}
\left(\mathbf{h}_{Y} \otimes C\right)\left(\phi^{\prime}\right) \eta(X, Y)(f) & =\left(\mathbf{h}_{Y} \otimes C\right)\left(\phi^{\prime}\right) \Phi_{Y}(X)\left(\varepsilon_{C} \otimes f\right) \\
& =\Phi_{Y}\left(X^{\prime}\right)\left(C^{*} \otimes \mathbf{h}_{Y}\right)\left(\phi^{\prime}\right)\left(\varepsilon_{C} \otimes f\right) \\
& =\Phi_{Y}\left(X^{\prime}\right)\left(\varepsilon_{C} \otimes f \phi^{\prime}\right)=\eta\left(X^{\prime}, Y\right)\left(\mathbf{h}_{Y}\left(\phi^{\prime}\right)(f)\right)
\end{aligned}
$$


for any $f \in \operatorname{Hom}_{\mathcal{D}}(X, Y)$. This shows that the following diagram commutes:

$$
\begin{array}{cc}
\mathbf{h}_{Y}(X) \stackrel{\eta(X, Y)}{\longrightarrow} & \mathbf{h}_{Y}(X) \otimes C \\
\mathbf{h}_{Y}\left(\phi^{\prime}\right) \downarrow & \downarrow\left(\mathbf{h}_{Y} \otimes C\right)\left(\phi^{\prime}\right) \\
\mathbf{h}_{Y}\left(X^{\prime}\right) \stackrel{\eta\left(X^{\prime}, Y\right)}{\longrightarrow} & \mathbf{h}_{Y}\left(X^{\prime}\right) \otimes C .
\end{array}
$$

Now using the naturality of $\Phi: C^{*} \otimes h \longrightarrow h \otimes C$, we also have

$$
\begin{aligned}
\left(X^{\prime} \mathbf{h} \otimes C\right)\left(\phi^{\prime \prime}\right) \eta\left(X^{\prime}, Y\right)(g) & =(h \otimes C)\left(\phi^{\prime \prime}\right) \Phi_{Y}\left(X^{\prime}\right)\left(\varepsilon_{C} \otimes g\right) \\
& =\Phi_{Y^{\prime}}\left(X^{\prime}\right)\left(C^{*} \otimes h\right)\left(\phi^{\prime \prime}\right)\left(\varepsilon_{C} \otimes g\right) \\
& =\Phi_{Y^{\prime}}\left(X^{\prime}\right)\left(\sum_{i=1}^{k} \varepsilon_{C}\left(d_{i}^{\psi}\right) d_{i}^{*} \otimes \phi_{\psi}^{\prime \prime} g\right) \\
& =\Phi_{Y^{\prime}}\left(X^{\prime}\right)\left(\sum_{i=1}^{k} \varepsilon_{C}\left(d_{i}\right) d_{i}^{*} \otimes \phi^{\prime \prime} g\right) \\
& =\Phi_{Y^{\prime}}\left(X^{\prime}\right)\left(\varepsilon_{C} \otimes \phi^{\prime \prime} g\right)=\eta\left(X^{\prime}, Y^{\prime}\right)\left(\phi^{\prime \prime} g\right) \\
& =\eta\left(X^{\prime}, Y^{\prime}\right)\left(X^{\prime} \mathbf{h}\left(\phi^{\prime \prime}\right)(g)\right)
\end{aligned}
$$

for any $g \in \mathbf{h}_{Y}\left(X^{\prime}\right)$. Thus, we get the following commutative diagram:

$$
\begin{array}{cc}
\mathbf{h}_{Y}\left(X^{\prime}\right) \stackrel{\eta\left(X^{\prime}, Y\right)}{\longrightarrow} & \mathbf{h}_{Y}\left(X^{\prime}\right) \otimes C \\
X^{\prime} \mathbf{h}\left(\phi^{\prime \prime}\right) \downarrow & \downarrow \downarrow\left(X^{\prime} \mathbf{h} \otimes C\right)\left(\phi^{\prime \prime}\right) \\
\mathbf{h}_{Y^{\prime}}\left(X^{\prime}\right) \stackrel{\eta\left(X^{\prime}, Y^{\prime}\right)}{\longrightarrow} & \mathbf{h}_{Y^{\prime}}\left(X^{\prime}\right) \otimes C .
\end{array}
$$

It now follows from (3.51) and (3.52) that the following diagram commutes:

$$
\begin{array}{ccc}
h(X, Y) & \stackrel{\eta(X, Y)}{\longrightarrow} & h(X, Y) \otimes C \\
h(\phi) \downarrow & \downarrow(h \otimes C)(\phi) \\
h\left(X^{\prime}, Y^{\prime}\right) \stackrel{\eta\left(X^{\prime}, Y^{\prime}\right)}{\longrightarrow} & h\left(X^{\prime}, Y^{\prime}\right) \otimes C .
\end{array}
$$

This shows that $\eta \in W_{2}$. It remains to check that $\gamma^{\prime}$ and $\delta^{\prime}$ are inverses of each other. First we verify that

$$
\left(\left(\delta^{\prime} \circ \gamma^{\prime}\right)(\eta)\right)(X, Y)=\eta(X, Y)
$$

for all $X, Y \in \operatorname{Ob}(\mathcal{D})$. For this, we set

$$
\Phi=\gamma^{\prime}(\eta) .
$$


Then, for any $f \in \operatorname{Hom}_{\mathcal{D}}(X, Y)$, we have

$$
\begin{aligned}
\left(\left(\delta^{\prime} \circ \gamma^{\prime}\right)(\eta)\right)(X, Y)(f) & =\Phi_{Y}(X)\left(\varepsilon_{C} \otimes f\right) \\
& =\left(\mathbf{h}_{Y} \otimes C\right)(f) \Phi_{Y}(Y)\left(\varepsilon_{C} \otimes \mathrm{id}_{Y}\right) \quad(\text { by }(3.49)) \\
& =\sum\left(\mathbf{h}_{Y} \otimes C\right)(f)\left(a_{Y} \otimes \varepsilon\left(c_{Y_{2}}\right) c_{Y_{1}}\right) \\
& =\sum\left(\mathbf{h}_{Y} \otimes C\right)(f)\left(a_{Y} \otimes c_{Y}\right) \\
& =\sum a_{Y} f_{\psi} \otimes c_{Y} \psi=\eta(X, Y)(f) \quad \text { (by Lemma 3.9). }
\end{aligned}
$$

Next, we will show that $\left(\left(\gamma^{\prime} \circ \delta^{\prime}\right)(\Phi)\right)_{Y}(X)=\Phi_{Y}(X)$ for any $X, Y \in \operatorname{Ob}(\mathcal{D})$. Since $C^{*} \otimes \mathbf{h}_{Y}(X)$ and $\mathbf{h}_{Y}(X) \otimes C$ are right $C$-comodules for any $X, Y \in \operatorname{Ob}(\mathcal{D})$, they are also left $C^{*}$-modules. The left actions are respectively given by

$$
\begin{aligned}
d^{*}\left(c^{*} \otimes f\right) & :=\sum_{i=1}^{k} d^{*}\left(d_{i}^{\psi}\right)\left(d_{i}^{*} \cdot c^{*}\right) \otimes f_{\psi}, \\
d^{*}(f \otimes x) & :=d^{*}\left(x_{2}\right)\left(f \otimes x_{1}\right),
\end{aligned}
$$

for any $d^{*}, c^{*} \in C^{*}, f \in \mathbf{h}_{Y}(X)$ and $x \in C$. Moreover, since

$$
\Phi_{Y}(X): C^{*} \otimes \mathbf{h}_{Y}(X) \longrightarrow \mathbf{h}_{Y}(X) \otimes C
$$

is right $C$-colinear, it is also left $C^{*}$-linear. We now set $\eta=\delta^{\prime}(\Phi)$. Then, for any $c^{*} \otimes f \in C^{*} \otimes \mathbf{h}_{Y}(X)$, we have

$$
\begin{aligned}
& \left(\left(\gamma^{\prime} \circ \delta^{\prime}\right)(\Phi)\right)_{Y}(X)\left(c^{*} \otimes f\right) \\
& =\left(\gamma^{\prime}(\eta)\right)_{Y}(X)\left(c^{*} \otimes f\right) \\
& =\sum a_{Y} f_{\psi} \otimes c^{*}\left(c_{Y_{2}}\right) c_{Y_{1}} \psi \\
& =\sum\left(\mathbf{h}_{Y} \otimes C\right)(f)\left(c^{*}\left(c_{Y_{2}}\right)\left(a_{Y} \otimes c_{Y_{1}}\right)\right) \\
& =\left(\mathbf{h}_{Y} \otimes C\right)(f)\left(c^{*}\left(\sum a_{Y} \otimes c_{Y}\right)\right) \\
& =\left(\mathbf{h}_{Y} \otimes C\right)(f)\left(c^{*}\left(\Phi_{Y}(Y)\left(\varepsilon_{C} \otimes \mathrm{id}_{Y}\right)\right)\right) \\
& =\left(\mathbf{h}_{Y} \otimes C\right)(f)\left(\Phi_{Y}(Y)\left(c^{*}\left(\varepsilon_{C} \otimes \mathrm{id}_{Y}\right)\right)\right) \quad\left(\text { since } \Phi_{Y}(X) \text { is } C^{*}\right. \text {-linear) } \\
& =\left(\mathbf{h}_{Y} \otimes C\right)(f) \Phi_{Y}(Y)\left(c^{*} \otimes \mathrm{id}_{Y}\right) \quad(\text { by }(3.53)) \\
& =\Phi_{Y}(X)\left(C^{*} \otimes \mathbf{h}_{Y}\right)(f)\left(c^{*} \otimes \mathrm{id}_{Y}\right) \quad\left(\Phi_{Y} \text { is a morphism of right } \mathcal{D} \text {-modules }\right) \\
& =\Phi_{Y}(X)\left(c^{*} \otimes f\right)
\end{aligned}
$$

This proves the result. 
Theorem 3.19: Let $(\mathcal{D}, C, \psi)$ be an entwining structure and assume that $C$ is a finite-dimensional coalgebra. Let $\mathscr{F}: \mathscr{M}(\psi)_{\mathcal{D}}^{C} \longrightarrow$ Mod-D be the functor forgetting the $C$-coaction and $\mathscr{G}: \operatorname{Mod}-\mathcal{D} \longrightarrow \mathscr{M}(\psi)_{\mathcal{D}}^{C}$ given by $\mathcal{N} \mapsto \mathcal{N} \otimes C$ be its right adjoint. Then, the following statements are equivalent:

(i) $(\mathscr{F}, \mathscr{G})$ is a Frobenius pair.

(ii) There exist $\eta \in W_{1}$ and $\theta \in V_{1}$ such that the corresponding morphisms $\gamma^{\prime}(\eta)=\Phi: C^{*} \otimes h \longrightarrow h \otimes C$ and $\alpha^{\prime}(\theta)=\Upsilon: h \otimes C \longrightarrow C^{*} \otimes h$ given by

$$
\begin{aligned}
& \Phi_{X Y}\left(c^{*} \otimes f\right)=\sum a_{Y} f_{\psi} \otimes c^{*}\left(c_{Y_{2}}\right) c_{Y_{1}}{ }^{\psi}, \\
& \Upsilon_{X Y}(f \otimes d)=\sum_{i=1}^{k} d_{i}^{*} \otimes f_{\psi} \circ \theta_{X}\left(d_{i}^{\psi} \otimes d\right),
\end{aligned}
$$

where $f \in \mathbf{h}_{Y}(X), c^{*} \in C^{*}$ and $d \in C$, are inverses of each other.

(iii) $C^{*} \otimes h$ and $h \otimes C$ are isomorphic as objects of the category ${ }_{\mathcal{D}} \mathscr{M}(\psi)_{\mathcal{D}}^{C}$ of functors from $\mathcal{D}$ to $\mathscr{M}(\psi)_{\mathcal{D}}^{C}$.

Proof. (i) $\Rightarrow$ (ii). By assumption, there exist $\eta \in W_{1}$ and $\theta \in V_{1}$ satisfying (3.31) and (3.32). Then, $\alpha^{\prime}(\theta)=\Upsilon$ and $\gamma^{\prime}(\eta)=\Phi$ are morphisms in ${ }_{\mathcal{D}} \mathscr{M}(\psi)_{\mathcal{D}}^{C}$ in the notation of Proposition 3.17 and Proposition 3.18. Since $\Upsilon_{X Y}: \mathbf{h}_{Y}(X) \otimes C \longrightarrow C^{*} \otimes \mathbf{h}_{Y}(X)$ and $\Phi_{X Y}: C^{*} \otimes \mathbf{h}_{Y}(X) \longrightarrow \mathbf{h}_{Y}(X) \otimes C$ are right $C$-colinear, they are also left $C^{*}$-linear. Using this fact and (3.53), we have

$$
\begin{aligned}
\Upsilon_{X Y} & \left(\Phi_{X Y}\left(c^{*} \otimes f\right)\right) \\
& =\Upsilon_{X Y}\left(\Phi_{X Y}\left(\left(C^{*} \otimes \mathbf{h}_{Y}\right)(f)\left(c^{*} \otimes \mathrm{id}_{Y}\right)\right)\right) \\
& =\Upsilon_{X Y}\left(\left(\mathbf{h}_{Y} \otimes C\right)(f)\left(\Phi_{Y Y}\left(c^{*} \otimes \operatorname{id}_{Y}\right)\right)\right) \\
& =\left(C^{*} \otimes \mathbf{h}_{Y}\right)(f)\left(\Upsilon_{Y Y}\left(\Phi_{Y Y}\left(c^{*} \otimes \operatorname{id}_{Y}\right)\right)\right) \\
& =\left(C^{*} \otimes \mathbf{h}_{Y}\right)(f)\left(\Upsilon_{Y Y}\left(\Phi_{Y Y}\left(c^{*} \bullet \varepsilon_{C} \otimes \operatorname{id}_{Y}\right)\right)\right) \\
& =\left(C^{*} \otimes \mathbf{h}_{Y}\right)(f)\left(c^{*} \cdot\left(\Upsilon_{Y Y}\left(\Phi_{Y Y}\left(\varepsilon_{C} \otimes \operatorname{id}_{Y}\right)\right)\right)\right) \\
& =\left(C^{*} \otimes \mathbf{h}_{Y}\right)(f)\left(c^{*} \cdot\left(\Upsilon_{Y Y}\left(\eta(Y, Y)\left(\operatorname{id}_{Y}\right)\right)\right)\right) \\
& =\left(C^{*} \otimes \mathbf{h}_{Y}\right)(f)\left(c^{*} \cdot\left(\sum_{i=1}^{k} \sum d_{i}^{*} \otimes\left(a_{Y}\right)_{\psi} \circ \theta_{X}\left(d_{i}^{\psi} \otimes c_{Y}\right)\right)\right) \\
& =\left(C^{*} \otimes \mathbf{h}_{Y}\right)(f)\left(c^{*} \cdot\left(\sum_{i=1}^{k} \varepsilon_{C}\left(d_{i}\right) d_{i}^{*} \otimes \operatorname{id}_{Y}\right)\right) \\
& =\left(C^{*} \otimes \mathbf{h}_{Y}\right)(f)\left(c^{*} \bullet \varepsilon_{C} \otimes \operatorname{id}_{Y}\right)=c^{*} \otimes f
\end{aligned}
$$

for any $c^{*} \otimes f \in C^{*} \otimes \mathbf{h}_{Y}(X)$. Thus, $\Upsilon \circ \Phi=\operatorname{id}_{C^{*} \otimes h}$. 
Using the naturality of $\Upsilon$ and $\Phi$, we have

$$
\begin{aligned}
\Phi_{X Y} & \left(\Upsilon_{X Y}(f \otimes c)\right) \\
& =\Phi_{X Y}\left(\Upsilon_{X Y}\left((h \otimes C)(f)(X)\left(\mathrm{id}_{X} \otimes c\right)\right)\right) \\
& =(h \otimes C)(f)(X)\left(\Phi_{X X}\left(\Upsilon_{X X}\left(\mathrm{id}_{X} \otimes c\right)\right)\right) \\
& =(h \otimes C)(f)(X)\left(\Phi_{X X}\left(\sum_{i=1}^{k} d_{i}^{*} \otimes \theta_{X}\left(d_{i} \otimes c\right)\right)\right) \\
& =(h \otimes C)(f)(X)\left(\sum_{i=1}^{k} \sum_{X}\left(\theta_{X}\left(d_{i} \otimes c\right)\right)_{\psi} \otimes d_{i}^{*}\left(c_{X_{2}}\right) c_{X_{1}} \psi\right) \\
& =(h \otimes C)(f)(X) \sum a_{X}\left(\theta_{X}\left(c_{X_{2}} \otimes c\right)\right)_{\psi} \otimes c_{X_{1}} \psi \\
& =(h \otimes C)(f)(X) \sum a_{X} \circ \theta_{X}\left(c_{X} \otimes c_{1}\right) \otimes c_{2} \\
& =(h \otimes C)(f)(X)\left(\varepsilon_{C}\left(c_{1}\right) \operatorname{id}_{X} \otimes c_{2}\right) \\
& =f \otimes c
\end{aligned}
$$

for any $f \otimes c \in \mathbf{h}_{Y}(X) \otimes C$. Thus, $\Phi \circ \Upsilon=\mathrm{id}_{h \otimes C}$. This proves (ii).

(ii) $\Rightarrow$ (iii) is obvious since both $\Phi$ and $\Upsilon$ are morphisms in $\mathcal{D} \mathscr{M}(\psi)_{\mathcal{D}}^{C}$.

(iii) $\Rightarrow\left(\right.$ i). Let $\Phi: C^{*} \otimes h \longrightarrow h \otimes C$ denote the isomorphism in ${ }_{\mathcal{D}} \mathscr{M}(\psi)_{\mathcal{D}}^{C}$. We consider the following morphism of $\left(\mathcal{D}^{o p} \otimes \mathcal{D}\right)$-modules:

$$
\Lambda: h \longrightarrow C^{*} \otimes h, \quad \Lambda_{Y}(X)(f):=\varepsilon_{C} \otimes f,
$$

for any $f \in \operatorname{Hom}_{\mathcal{D}}(X, Y)$. We now set

$$
\eta=\Phi \circ \Lambda \in W_{1} \quad \text { and } \quad \theta=\beta^{\prime}\left(\Phi^{-1}\right) \in V_{1}
$$

where $\beta^{\prime}$ is as in Proposition 3.17. If

$$
\eta(X, Y)(f)=\sum \hat{f} \otimes c_{f},
$$

then

$$
\begin{aligned}
\varepsilon_{C} \otimes f & =\Phi_{X Y}^{-1}\left(\Phi_{X Y}\left(\varepsilon_{C} \otimes f\right)\right) \\
& =\Phi_{X Y}^{-1}(\eta(X, Y)(f)) \\
& =\sum \Phi_{X Y}^{-1}\left(\hat{f} \otimes c_{f}\right) \\
& =\sum\left(\alpha^{\prime}(\theta)\right)_{X Y}\left(\hat{f} \otimes c_{f}\right) \\
& =\sum \sum_{i=1}^{k} d_{i}^{*} \otimes \hat{f}_{\psi} \circ \theta_{X}\left(d_{i}^{\psi} \otimes c_{f}\right) \quad(\text { by }(3.46)) .
\end{aligned}
$$


Using the isomorphism as in (3.38) and evaluating the equality in (3.55) at $d \in C$, we get (3.32). We also have

$$
\begin{aligned}
\operatorname{id}_{X} & \otimes d \\
& =\Phi_{X X}\left(\Phi_{X X}^{-1}\left(\operatorname{id}_{X} \otimes d\right)\right) \\
& =\Phi_{X X}\left(\left(\alpha^{\prime}(\theta)\right)_{X X}\left(\operatorname{id}_{X} \otimes d\right)\right) \\
& =\sum_{i=1}^{k} \Phi_{X X}\left(d_{i}^{*} \otimes \theta_{X}\left(d_{i} \otimes d\right)\right) \\
& =\sum_{i=1}^{k} \Phi_{X X}\left(\left(C^{*} \otimes \mathbf{h}_{X}\right)\left(\theta_{X}\left(d_{i} \otimes d\right)\right)\left(d_{i}^{*} \otimes \operatorname{id}_{X}\right)\right) \\
& =\sum_{i=1}^{k}\left(\mathbf{h}_{X} \otimes C\right)\left(\theta_{X}\left(d_{i} \otimes d\right)\right)\left(\Phi_{X X}\left(d_{i}^{*} \otimes \operatorname{id}_{X}\right)\right) \\
& =\sum_{i=1}^{k}\left(\mathbf{h}_{X} \otimes C\right)\left(\theta_{X}\left(d_{i} \otimes d\right)\right)\left(\Phi_{X X}\left(d_{i}^{*} \cdot\left(\varepsilon_{C} \otimes \operatorname{id}_{X}\right)\right)\right) \\
& =\sum_{i=1}^{k}\left(\mathbf{h}_{X} \otimes C\right)\left(\theta_{X}\left(d_{i} \otimes d\right)\right)\left(d_{i}^{*} \cdot \Phi_{X X}\left(\varepsilon_{C} \otimes \operatorname{id}_{X}\right)\right)
\end{aligned}
$$

(since $\Phi_{X X}$ is $C^{*}$-linear)

$$
\begin{aligned}
& =\sum_{i=1}^{k}\left(\mathbf{h}_{X} \otimes C\right)\left(\theta_{X}\left(d_{i} \otimes d\right)\right)\left(d_{i}^{*} \cdot\left(\eta(X, X)\left(\operatorname{id}_{X}\right)\right)\right) \\
& =\sum_{i=1}^{k} \sum\left(\mathbf{h}_{X} \otimes C\right)\left(\theta_{X}\left(d_{i} \otimes d\right)\right)\left(d_{i}^{*} \cdot\left(a_{X} \otimes c_{X}\right)\right) \\
& =\sum_{i=1}^{k} \sum\left(\mathbf{h}_{X} \otimes C\right)\left(\theta_{X}\left(d_{i} \otimes d\right)\right)\left(d_{i}^{*}\left(c_{X_{2}}\right)\left(a_{X} \otimes c_{X_{1}}\right)\right) \\
& =\sum\left(\mathbf{h}_{X} \otimes C\right)\left(\theta_{X}\left(c_{X_{2}} \otimes d\right)\right)\left(a_{X} \otimes c_{X_{1}}\right) \\
& =\sum a_{X} \circ\left(\theta_{X}\left(c_{X_{2}} \otimes d\right)\right)_{\psi} \otimes c_{X_{1}}{ }^{k} \\
& =\sum a_{X} \circ \theta_{X}\left(c_{X} \otimes d_{1}\right) \otimes d_{2}
\end{aligned}
$$

By applying the map $\operatorname{id}_{\mathbf{h}_{X}(X)} \otimes \varepsilon_{C}$, we obtain

$$
\varepsilon_{C}(d) \cdot \operatorname{id}_{X}=\sum a_{X}\left(\theta_{X}\left(c_{X} \otimes d\right)\right) .
$$


Now using Lemma 3.9 and (3.56), we obtain

$$
\begin{aligned}
\sum \hat{f} \otimes\left(\theta_{X}\left(c_{f} \otimes d\right)\right) & =\sum\left(\operatorname{id}_{\mathbf{h}_{Y}(X)} \otimes \theta_{X}\right)\left(\hat{f} \otimes c_{f} \otimes d\right) \\
& =\left(\operatorname{id}_{\mathbf{h}_{Y}(X)} \otimes \theta_{X}\right)(\eta(X, Y)(f) \otimes d) \\
& =\sum\left(\operatorname{id}_{\mathbf{h}_{Y}(X)} \otimes \theta_{X}\right)\left(f a_{X} \otimes c_{X} \otimes d\right)
\end{aligned}
$$

for any $f \in \operatorname{Hom}_{\mathcal{D}}(X, Y)$ and $d \in C$. Applying to both sides the composition $\operatorname{Hom}_{\mathcal{D}}(X, Y) \otimes \operatorname{Hom}_{\mathcal{D}}(X, X) \longrightarrow \operatorname{Hom}_{\mathcal{D}}(X, Y)$, we obtain

$$
\sum \hat{f} \circ\left(\theta_{X}\left(c_{f} \otimes d\right)\right)=\sum f a_{X} \circ \theta_{X}\left(c_{X} \otimes d\right)=\varepsilon_{C}(d) f .
$$

This proves $(3.31)$. Therefore, $(\mathscr{F}, \mathscr{G})$ is a Frobenius pair by Theorem 3.14. This completes the proof.

\section{Categorical Galois extensions and entwining structures}

Let $\mathcal{D}$ be a small $K$-linear category. Let $(\mathcal{D}, C, \psi)$ be a right-right entwining structure. We denote by $\mathcal{D} \mathscr{M}_{\mathcal{D}}$ the category of $\mathcal{D}$ - $\mathcal{D}$ bimodules, i.e., the category whose objects are functors from $\mathcal{D}^{o p} \otimes \mathcal{D}$ to Vect ${ }_{K}$ and whose morphisms are natural transformations between these functors. We recall the functors $h$ and $h \otimes C$ in $\mathcal{D}_{\mathcal{D}}$ from (3.20) and (3.21) respectively:

$$
h(X, Y)=\operatorname{Hom}_{\mathcal{D}}(X, Y), \quad(h(\phi))(f)=\phi^{\prime \prime} f \phi^{\prime},
$$

$(4.2)(h \otimes C)(X, Y)=\operatorname{Hom}_{\mathcal{D}}(X, Y) \otimes C, \quad((h \otimes C)(\phi))(f \otimes c)=\phi^{\prime \prime} f \phi_{\psi}^{\prime} \otimes c^{\psi}$,

for any $(X, Y) \in \operatorname{Ob}\left(\mathcal{D}^{o p} \otimes \mathcal{D}\right), \phi:=\left(\phi^{\prime}, \phi^{\prime \prime}\right) \in \operatorname{Hom}_{\mathcal{D}^{o p} \otimes \mathcal{D}}\left((X, Y),\left(X^{\prime}, Y^{\prime}\right)\right)$ and $f \in \operatorname{Hom}_{\mathcal{D}}(X, Y), c \in C$. We refer, for instance, to [19, §2.2] for the tensor product which makes $\mathcal{D} \mathscr{M}_{\mathcal{D}}$ a monoidal category with $h \in \mathcal{D}_{\mathcal{D}}$ as the unit object.

Definition 4.1: A $\mathcal{D}$-coring $\mathscr{C}$ is a coalgebra object in the monoidal category $\mathcal{D} \mathscr{M}_{\mathcal{D}}$. Explicitly, a $\mathcal{D}$-coring is a functor $\mathscr{C}: \mathcal{D}^{o p} \otimes \mathcal{D} \longrightarrow$ Vect $_{K}$ with two morphisms

$$
\Delta_{\mathscr{C}}: \mathscr{C} \longrightarrow \mathscr{C} \otimes_{\mathcal{D}} \mathscr{C}, \quad \varepsilon_{\mathscr{C}}: \mathscr{C} \longrightarrow h,
$$

satisfying the coassociativity and counit axioms in $\mathcal{D}_{\mathcal{D}}$. A right $\mathscr{C}$-comodule consists of a right $\mathcal{D}$-module $\mathcal{M}$ equipped with a morphism $\rho_{\mathcal{M}}: \mathcal{M} \longrightarrow \mathcal{M} \otimes_{\mathcal{D}} \mathscr{C}$ of right $\mathcal{D}$-modules satisfying

$$
\left(\operatorname{id}_{\mathcal{M}} \otimes_{\mathcal{D}} \Delta_{\mathscr{C}}\right) \circ \rho_{\mathcal{M}}=\left(\rho_{\mathcal{M}} \otimes_{\mathcal{D}} \operatorname{id}_{\mathscr{C}}\right) \circ \rho_{\mathcal{M}}, \quad\left(\operatorname{id}_{\mathcal{M}} \otimes_{\mathcal{D}} \varepsilon_{\mathscr{C}}\right) \circ \rho_{\mathcal{M}}=\operatorname{id}_{\mathcal{M}}
$$


A morphism $\eta:\left(\mathcal{M}, \rho_{\mathcal{M}}\right) \longrightarrow\left(\mathcal{N}, \rho_{\mathcal{N}}\right)$ of right $\mathscr{C}$-comodules is a morphism $\eta: \mathcal{M} \longrightarrow \mathcal{N}$ of right $\mathcal{D}$-modules satisfying

$$
\rho_{\mathcal{N}} \circ \eta=\left(\eta \otimes_{\mathcal{D}} \operatorname{id}_{\mathscr{C}}\right) \circ \rho_{\mathcal{M}} .
$$

The category of right $\mathscr{C}$-comodules will be denoted by Comod- $\mathscr{C}$.

Lemma 4.2: Let $(\mathcal{D}, C, \psi)$ be a right-right entwining structure. Then, the functor $h \otimes C$ is a $\mathcal{D}$-coring.

Proof. It may be verified that $(h \otimes C) \otimes_{\mathcal{D}}(h \otimes C) \cong h \otimes C \otimes C$. This gives us morphisms

$$
\begin{aligned}
\operatorname{id}_{h} \otimes \Delta_{C} & : h \otimes C \longrightarrow h \otimes C \otimes C \cong(h \otimes C) \otimes_{\mathcal{D}}(h \otimes C), \\
\operatorname{id}_{h} \otimes \varepsilon_{C} & : h \otimes C \longrightarrow h,
\end{aligned}
$$

in $\mathcal{D} \mathscr{M}_{\mathcal{D}}$. Using the coassociativity and counitality of the $K$-coalgebra $C$, it may be verified that $\operatorname{id}_{h} \otimes \Delta_{C}$ and $\operatorname{id}_{h} \otimes \varepsilon_{C}$ satisfy the coassociativity and counit axioms in the category $\mathcal{D}_{\mathcal{D}}$. Thus, $h \otimes C$ is a coalgebra object in $\mathcal{D} \mathscr{M}_{\mathcal{D}}$.

Proposition 4.3: Let $(\mathcal{D}, C, \psi)$ be a right-right entwining structure. Then, the category $\mathscr{M}(\psi)_{\mathcal{D}}^{C}$ of entwined modules is identical to the category Comod- $(h \otimes C)$.

Proof. Let $\mathcal{M} \in \mathscr{M}(\psi)_{\mathcal{D}}^{C}$. It may be verified that $\mathcal{M} \otimes C \cong \mathcal{M} \otimes_{\mathcal{D}}(h \otimes C)$ as right $\mathcal{D}$-modules. Then, by Lemma $2.4, \mathcal{M} \otimes C \in \mathscr{M}(\psi)_{\mathcal{D}}^{C}$ and we have

$$
\rho_{\mathcal{M}(X)}(\mathcal{M}(f)(m))=\mathcal{M}\left(f_{\psi}\right)\left(m_{0}\right) \otimes m_{1}{ }^{\psi}=(\mathcal{M} \otimes C)(f)\left(m_{0} \otimes m_{1}\right)
$$

for any $f \in \operatorname{Hom}_{\mathcal{D}}(X, Y)$ and $m \in \mathcal{M}(Y)$. We thus obtain a morphism

$$
\rho_{\mathcal{M}}: \mathcal{M} \longrightarrow \mathcal{M} \otimes C \cong \mathcal{M} \otimes_{\mathcal{D}}(h \otimes C)
$$

of right $\mathcal{D}$-modules given by

$$
\rho_{\mathcal{M}}(X):=\rho_{\mathcal{M}(X)}
$$

for each $X \in \mathrm{Ob}(\mathcal{D})$.

Applying (4.4), we have

$$
\begin{aligned}
& \mathcal{M} \otimes_{\mathcal{D}}(h \otimes C) \stackrel{\operatorname{id} \mathcal{M} \otimes_{\mathcal{D}} \Delta_{(h \otimes C)}=\mathrm{id}_{\mathcal{M}} \otimes \mathrm{id}_{h} \otimes \Delta_{C}}{\longrightarrow} \mathcal{M} \otimes_{\mathcal{D}}(h \otimes C) \otimes_{\mathcal{D}}(h \otimes C) \\
& \cong \downarrow \quad \cong \downarrow \\
& \mathcal{M} \otimes C \quad \stackrel{\operatorname{id} \mathcal{M} \otimes \Delta_{C}}{\longrightarrow} \quad \mathcal{M} \otimes C \otimes C
\end{aligned}
$$


and

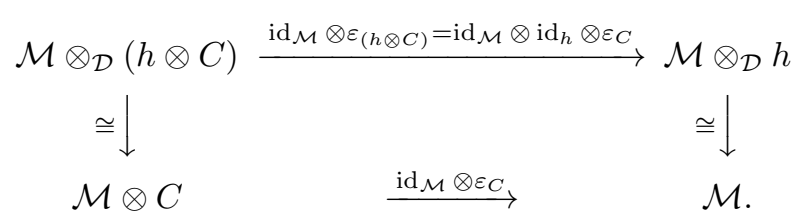

The conditions in (4.3) now follow from the fact that $\rho_{\mathcal{M}(X)}$ is a $C$-coaction for each $X \in \operatorname{Ob}(\mathcal{D})$. Therefore, $\mathcal{M}$ is a right $(h \otimes C)$-comodule.

Conversely, let $\mathcal{N} \in \operatorname{Comod}-(h \otimes C)$. Then, $\mathcal{N}$ is a right $\mathcal{D}$-module with a given morphism $\rho_{\mathcal{N}}: \mathcal{N} \longrightarrow \mathcal{N} \otimes_{\mathcal{D}}(h \otimes C) \cong \mathcal{N} \otimes C$ of right $\mathcal{D}$-modules satisfying the conditions in (4.3). Thus, for each $Y \in \operatorname{Ob}(\mathcal{D})$, we have a morphism $\rho_{\mathcal{N}}(Y): \mathcal{N}(Y) \longrightarrow \mathcal{N}(Y) \otimes C$ which satisfies

$$
\begin{aligned}
\left(\operatorname{id}_{\mathcal{N}(Y)} \otimes \Delta_{C}\right) \circ \rho_{\mathcal{N}}(Y) & =\left(\rho_{\mathcal{N}}(Y) \otimes \operatorname{id}_{C}\right) \circ \rho_{\mathcal{N}}(Y), \\
\left(\operatorname{id}_{\mathcal{N}}(Y) \otimes \varepsilon_{C}\right) \circ \rho_{\mathcal{N}}(Y) & =\operatorname{id}_{\mathcal{N}(Y) .}
\end{aligned}
$$

In (4.7), we have identified $\operatorname{id}_{\mathcal{N}} \otimes \Delta_{C}=\operatorname{id}_{\mathcal{N}} \otimes \Delta_{h \otimes C}$ and $\operatorname{id}_{\mathcal{N}} \otimes \varepsilon_{C}=\operatorname{id}_{\mathcal{N}} \otimes \varepsilon_{(h \otimes C)}$ as in (4.5) and (4.6) respectively. Therefore, $\rho_{\mathcal{N}}(Y)$ defines a right $C$-comodule structure on $\mathcal{N}(Y)$ for every $Y \in \operatorname{Ob}(\mathcal{D})$. Since $\rho_{\mathcal{N}}$ is a morphism of right $\mathcal{D}$-modules, we also have

$$
\rho_{\mathcal{N}}(X)(\mathcal{N}(f)(n))=(\mathcal{N} \otimes C)(f)\left(n_{0} \otimes n_{1}\right)=\mathcal{N}\left(f_{\psi}\right)\left(n_{0}\right) \otimes n_{1}{ }^{\psi}
$$

for any $f \in \operatorname{Hom}_{\mathcal{D}}(X, Y)$ and $n \in \mathcal{N}(Y)$. Therefore, $\mathcal{N} \in \mathscr{M}(\psi)_{\mathcal{D}}^{C}$.

Lemma 4.4: Let $i: \mathcal{E} \longrightarrow \mathcal{D}$ be an inclusion of small $K$-linear categories. Then, the functor $h \otimes_{\mathcal{E}} h: \mathcal{E}^{o p} \otimes \mathcal{E} \longrightarrow$ Vect $_{K}$ is a $\mathcal{D}$-coring, where $h$ is the $\mathcal{D}$-D-bimodule as in (4.1).

Proof. It is immediate that the functor $h \otimes_{\mathcal{E}} h$ is a $\mathcal{D}$ - $\mathcal{D}$-bimodule. We need to show that $h \otimes_{\mathcal{E}} h$ is a coalgebra object in $\mathcal{D} \mathscr{M}_{\mathcal{D}}$. We now define

$$
\Delta: h \otimes_{\mathcal{E}} h \longrightarrow\left(h \otimes_{\mathcal{E}} h\right) \otimes_{\mathcal{D}}\left(h \otimes_{\mathcal{E}} h\right) \cong\left(h \otimes_{\mathcal{E}} h\right) \otimes_{\mathcal{E}} h
$$

as follows: for $(X, Y) \in \operatorname{Ob}\left(\mathcal{D}^{o p} \otimes \mathcal{D}\right)$, we set

$$
\begin{gathered}
\Delta(X, Y): \mathbf{h}_{Y} \otimes_{\mathcal{E} X} \mathbf{h} \longrightarrow\left(h \otimes_{\mathcal{E}} h\right)(-, Y) \otimes_{\mathcal{E}} h(X,-) \cong \mathbf{h}_{Y} \otimes_{\mathcal{E}} h \otimes_{\mathcal{E} X} \mathbf{h}, \\
f \otimes f^{\prime} \mapsto f \otimes \operatorname{id}_{Z} \otimes f^{\prime},
\end{gathered}
$$

for any $f \otimes f^{\prime} \in \mathbf{h}_{Y}(Z) \otimes_{X} \mathbf{h}(Z)$ and $Z \in \mathrm{Ob}(\mathcal{E})$. It is easy to check that $\Delta(X, Y)$ is well-defined. Also, it can be verified that for any morphism

$$
\left(\phi^{\prime}, \phi^{\prime \prime}\right):(X, Y) \longrightarrow\left(X^{\prime}, Y^{\prime}\right)
$$


in $\mathcal{D}^{o p} \otimes \mathcal{D}$, the following diagram commutes:

$$
\begin{array}{ccc}
\mathbf{h}_{Y} \otimes_{\mathcal{E} X} \mathbf{h} \stackrel{\Delta(X, Y)}{\longrightarrow} & \mathbf{h}_{Y} \otimes_{\mathcal{E}} h \otimes_{\mathcal{E} X} \mathbf{h} \\
h_{\phi^{\prime \prime}} \otimes_{\mathcal{E}_{\phi^{\prime}}} h \mid & \\
\mathbf{h}_{Y^{\prime}} \otimes_{\mathcal{E} X^{\prime}} \mathbf{h} \stackrel{\Delta\left(X^{\prime}, Y^{\prime}\right)}{\longrightarrow} \mathbf{h}_{Y^{\prime}} \otimes_{\mathcal{E}} h \otimes_{\mathcal{E} X^{\prime}} \mathbf{h} .
\end{array}
$$

Thus, $\Delta$ is a morphism of $\mathcal{D}$-D -bimodules. The map $\varepsilon: h \otimes_{\mathcal{E}} h \longrightarrow h$ is defined by composition. It may be verified that $\Delta$ and $\varepsilon$ satisfy the coassociativity and counit axioms respectively.

Let $\mathcal{D}$ be a small $K$-linear category and let $C$ be a $K$-coalgebra. We consider the category $\mathcal{D} \mathscr{M}^{C}$ of left-right Doi-Hopf modules (compare Example 2.3). Explicitly, an object in $\mathcal{D} \mathscr{M}^{C}$ consists of a left $\mathcal{D}$-module $\mathcal{M}$ with a given right $C$-comodule structure on $\mathcal{M}(X)$ for each $X \in \operatorname{Ob}(\mathcal{D})$ such that the following compatibility condition holds:

$$
(\mathcal{M}(f)(m))_{0} \otimes(\mathcal{M}(f)(m))_{1}=\mathcal{M}(f)\left(m_{0}\right) \otimes m_{1}
$$

for each $f \in \operatorname{Hom}_{\mathcal{D}}(X, Y)$ and $m \in \mathcal{M}(X)$. A morphism $\eta: \mathcal{M} \longrightarrow \mathcal{N}$ in $\mathcal{D} \mathscr{M}^{C}$ is a left $\mathcal{D}$-module morphism such that each $\eta(X): \mathcal{M}(X) \longrightarrow \mathcal{N}(X)$ is right $C$-colinear. By definition, $(h \otimes C)(X,-)={ }_{X} \mathbf{h} \otimes C$ is a left $\mathcal{D}$-module for each $X \in \operatorname{Ob}(\mathcal{D})$. The map id $\otimes \Delta_{C}: \operatorname{Hom}_{\mathcal{D}}(X, Y) \otimes C \longrightarrow \operatorname{Hom}_{\mathcal{D}}(X, Y) \otimes C \otimes C$ gives a right $C$-comodule structure on $\left({ }_{X} \mathbf{h} \otimes C\right)(Y)$ for each $Y \in \mathrm{Ob}(\mathcal{D})$. Clearly, ${ }_{X} \mathbf{h} \otimes C \in \mathcal{D}^{C}$.

From this point onwards, we suppose additionally that each $\operatorname{Hom}_{\mathcal{D}}(X, Y)$ has a given right $C$-comodule structure denoted by

$$
\rho_{X Y}: \operatorname{Hom}_{\mathcal{D}}(X, Y) \longrightarrow \operatorname{Hom}_{\mathcal{D}}(X, Y) \otimes C .
$$

Definition 4.5: Let $\mathcal{E} \subseteq \mathcal{D}$ be the subcategory with $\mathrm{Ob}(\mathcal{E})=\mathrm{Ob}(\mathcal{D})$ and

$$
\begin{aligned}
& \operatorname{Hom}_{\mathcal{E}}(X, Y)= \operatorname{Hom}_{\text {Mod -D }}^{C}\left(\mathbf{h}_{X}, \mathbf{h}_{Y}\right) \\
&=\left\{\eta \in \operatorname{Hom}_{M o d-\mathcal{D}}\left(\mathbf{h}_{X}, \mathbf{h}_{Y}\right) \mid \eta \text { is objectwise } C \text {-colinear }\right\} \\
&=\left\{g \in \operatorname{Hom}_{\mathcal{D}}(X, Y) \mid \rho_{Z Y}(g f)=\left({ }_{Z} \mathbf{h} \otimes C\right)(g)\left(\rho_{Z X}(f)\right)\right. \\
&\left.\qquad \forall f \in \operatorname{Hom}_{\mathcal{D}}(Z, X)\right\} .
\end{aligned}
$$

We will say that $\mathcal{E}$ is the subcategory of $C$-coinvariants of $\mathcal{D}$. 
Example 4.6: Let $H$ be a Hopf algebra over $K$ and let $\mathcal{D}$ be a right co- $H$ category. In this case, the subcategory $\mathcal{E}$ of $H$-coinvariants of $\mathcal{D}$ is given by setting

$$
\operatorname{Ob}(\mathcal{E})=\operatorname{Ob}(\mathcal{D}) \quad \text { and } \quad \operatorname{Hom}_{\mathcal{E}}(X, Y)=\operatorname{Hom}_{\mathcal{D}}(X, Y)^{c o H} .
$$

It follows that the right $C$-comodule structures

$$
\rho_{X Y}: \operatorname{Hom}_{\mathcal{D}}(X, Y) \longrightarrow \operatorname{Hom}_{\mathcal{D}}(X, Y) \otimes C
$$

induce a morphism ${ }_{X} \mathbf{h} \longrightarrow{ }_{X} \mathbf{h} \otimes C$ of left $\mathcal{E}$-modules for each $X \in \mathrm{Ob}(\mathcal{D})$. Further, for every $Y \in \mathrm{Ob}(\mathcal{D})$, this induces a morphism

$$
\begin{gathered}
\left(h \otimes_{\mathcal{E} X} \mathbf{h}\right)(Y)=\mathbf{h}_{Y} \otimes_{\mathcal{E} X} \mathbf{h} \longrightarrow \mathbf{h}_{Y} \otimes_{\mathcal{E} X} \mathbf{h} \otimes C=\left(h \otimes_{\mathcal{E} X} \mathbf{h}\right)(Y) \otimes C, \\
f \otimes f^{\prime} \mapsto f \otimes \rho_{X Z}\left(f^{\prime}\right),
\end{gathered}
$$

where $f \in \operatorname{Hom}_{\mathcal{D}}(Z, Y), f^{\prime} \in \operatorname{Hom}_{\mathcal{D}}(X, Z)$ and $Z \in \operatorname{Ob}(\mathcal{E})$. It may be easily verified that the coaction in (4.10) makes $h \otimes_{\mathcal{E} X} \mathbf{h}$ an object of ${ }_{\mathcal{D}} \mathscr{M}^{C}$.

We obtain therefore canonical morphisms of $K$-vector spaces given by the following composition:

$$
\begin{aligned}
\left\{\operatorname{can}_{X Y}: \mathbf{h}_{Y} \otimes_{\mathcal{E} X} \mathbf{h}\right. & \longrightarrow \mathbf{h}_{Y} \otimes_{\mathcal{E}}\left({ }_{X} \mathbf{h} \otimes C\right) \\
& \left.\longrightarrow \mathbf{h}_{Y} \otimes_{\mathcal{D}}\left({ }_{X} \mathbf{h} \otimes C\right) \cong \operatorname{Hom}_{\mathcal{D}}(X, Y) \otimes C\right\}_{(X, Y) \in \mathrm{Ob}(\mathcal{D})^{2}} .
\end{aligned}
$$

For each $X \in \operatorname{Ob}(\mathcal{D})$, this induces a morphism in $\mathcal{D} \mathscr{M}^{C}$ as follows:

$$
\operatorname{can}_{X}: h \otimes_{\mathcal{E} X} \mathbf{h} \longrightarrow{ }_{X} \mathbf{h} \otimes C, \quad \operatorname{can}_{X}(Y):=\operatorname{can}_{X Y} .
$$

Definition 4.7: Let $C$ be a $K$-coalgebra and $\mathcal{D}$ be a small $K$-linear category such that $\operatorname{Hom}_{\mathcal{D}}(X, Y)$ has a right $C$-comodule structure for every $X, Y \in \operatorname{Ob}(\mathcal{D})$. Let $\mathcal{E}$ be a $K$-linear subcategory of $\mathcal{D}$. Then, $\mathcal{D}$ is called a $C$-Galois extension of $\mathcal{E}$ if:

(i) $\operatorname{Ob}(\mathcal{E})=\operatorname{Ob}(\mathcal{D})$ and $\operatorname{Hom}_{\mathcal{E}}(X, Y)=H o m_{\operatorname{Mod}-\mathcal{D}}^{C}\left(\mathbf{h}_{X}, \mathbf{h}_{Y}\right)$.

(ii) The induced canonical morphism $\operatorname{can}_{X}: h \otimes_{\mathcal{E} X} \mathbf{h} \longrightarrow{ }_{X} \mathbf{h} \otimes C$ is an isomorphism in $\mathcal{D} \mathscr{M}^{C}$ for each $X \in \mathrm{Ob}(\mathcal{D})$.

Let $\mathcal{D}$ be a $C$-Galois extension of $\mathcal{E}$. For each $X \in \mathrm{Ob}(\mathcal{D})$, we define

$$
\tau_{X}: C \longrightarrow \mathbf{h}_{X} \otimes_{\mathcal{E} X} \mathbf{h}, \quad \tau_{X}(c):=\operatorname{can}_{X X}^{-1}\left(\operatorname{id}_{X} \otimes c\right) .
$$

We refer to these as the translation maps of the Galois extension. 
Lemma 4.8: Let $\mathcal{D}$ be a $C$-Galois extension of $\mathcal{E}$. Let

$$
\left\{\tau_{X}: C \longrightarrow \mathbf{h}_{X} \otimes_{\mathcal{E} X} \mathbf{h}\right\}_{X \in \mathrm{Ob}(\mathcal{D})}
$$

be the associated translation maps. We use the notation $\tau_{X}(c)=c^{(1)} \otimes c^{(2)}$ (summation omitted). Then,

(i) $\tau_{X}$ is right $C$-colinear i.e., $c^{(1)} \otimes c^{(2)}{ }_{0} \otimes c^{(2)}{ }_{1}=\left(c_{1}\right)^{(1)} \otimes\left(c_{1}\right)^{(2)} \otimes c_{2}$.

(ii) For any $f \in \operatorname{Hom}_{\mathcal{D}}(X, Y)$, we have

$$
f_{0}\left(f_{1}\right)^{(1)} \otimes\left(f_{1}\right)^{(2)}=\operatorname{id}_{Y} \otimes f \in \mathbf{h}_{Y} \otimes \mathcal{E} X \mathbf{h} .
$$

(iii) $c^{(1)} c^{(2)}=\varepsilon_{C}(c) \cdot \operatorname{id}_{X}$.

Proof. The $C$-colinearity of $\tau_{X}$ follows from the $C$-colinearity of $\operatorname{can}_{X X}^{-1}$. Explicitly, for any $c \in C$, we have

$$
\begin{aligned}
c^{(1)} \otimes c_{0}^{(2)} \otimes c_{1}^{(2)}{ }_{1} & =(\operatorname{id} \otimes \rho) \tau_{X}(c)=(\operatorname{id} \otimes \rho) \operatorname{can}_{X X}^{-1}\left(\operatorname{id}_{X} \otimes c\right) \\
& =\left(\operatorname{can}_{X X}^{-1} \otimes \operatorname{id}_{C}\right)\left(\mathrm{id} \otimes \Delta_{C}\right)\left(\operatorname{id}_{X} \otimes c\right) \\
& =\left(\operatorname{can}_{X X}^{-1} \otimes \operatorname{id}_{C}\right)\left(\operatorname{id}_{X} \otimes c_{1} \otimes c_{2}\right) \\
& =\operatorname{can}_{X X}^{-1}\left(\operatorname{id}_{X} \otimes c_{1}\right) \otimes c_{2}=\tau_{X}\left(c_{1}\right) \otimes c_{2} \\
& =\left(c_{1}\right)^{(1)} \otimes\left(c_{1}\right)^{(2)} \otimes c_{2} .
\end{aligned}
$$

This proves (i). Since $\operatorname{can}_{X}^{-1}:{ }_{X} \mathbf{h} \otimes C \longrightarrow h \otimes_{\mathcal{E} X} \mathbf{h}$ is a morphism of left $\mathcal{D}$-modules for each $X \in \operatorname{Ob}(\mathcal{D})$, we also have

$$
\begin{aligned}
f_{0}\left(f_{1}\right)^{(1)} \otimes\left(f_{1}\right)^{(2)} & =\left(h \otimes_{\mathcal{E} X} \mathbf{h}\right)\left(f_{0}\right)\left(\tau_{X}\left(f_{1}\right)\right) \\
& =\left(h \otimes_{\mathcal{E} X} \mathbf{h}\right)\left(f_{0}\right)\left(\operatorname{can}_{X X}^{-1}\left(\operatorname{id}_{X} \otimes f_{1}\right)\right) \\
& =\operatorname{can}_{X Y}^{-1}\left((X \mathbf{h} \otimes C)\left(f_{0}\right)\left(\operatorname{id}_{X} \otimes f_{1}\right)\right) \\
& =\operatorname{can}_{X Y}^{-1}\left(f_{0} \otimes f_{1}\right)=\operatorname{id}_{Y} \otimes f .
\end{aligned}
$$

This proves (ii). Again using the definition of $\operatorname{can}_{X X}$ and $\tau_{X}$, we have

$$
\left(\operatorname{can}_{X X} \circ \tau_{X}\right)(c)=\operatorname{id}_{X} \otimes c .
$$

Thus,

$$
\operatorname{can}_{X X}\left(c^{(1)} \otimes c^{(2)}\right)=c^{(1)} c^{(2)} \otimes c^{(2)}{ }_{1}=\mathrm{id}_{X} \otimes c
$$

Now, by applying the map $i d \otimes \varepsilon_{C}$ to both sides, we get (iii). 
Theorem 4.9: Let $\mathcal{D}$ be a $C$-Galois extension of $\mathcal{E}$. We denote by

$$
\rho_{X Y}: \operatorname{Hom}_{\mathcal{D}}(X, Y) \longrightarrow \operatorname{Hom}_{\mathcal{D}}(X, Y) \otimes C
$$

the right $C$-comodule structure maps. Then, there exists a unique right-right entwining structure $(\mathcal{D}, C, \psi)$ which makes $\mathbf{h}_{Y}$ an object in $\mathscr{M}(\psi)_{\mathcal{D}}^{C}$ for every $Y \in \operatorname{Ob}(\mathcal{D})$ with its canonical $\mathcal{D}$-module structure and right $C$-coactions $\left\{\rho_{X Y}\right\}_{X \in \mathrm{Ob}(\mathcal{D})}$.

This entwining structure $(\mathcal{D}, C, \psi)$ is given by

$$
\begin{aligned}
\psi_{X Y}: C \otimes \operatorname{Hom}_{\mathcal{D}}(X, Y) \stackrel{\tau_{Y} \otimes \mathrm{id}}{\longrightarrow} & \mathbf{h}_{Y} \otimes_{\mathcal{E} Y} \mathbf{h} \otimes \operatorname{Hom}_{\mathcal{D}}(X, Y) \\
& \longrightarrow \mathbf{h}_{Y} \otimes_{\mathcal{E} X} \mathbf{h} \stackrel{\operatorname{can}_{X Y}}{\longrightarrow} \operatorname{Hom}_{\mathcal{D}}(X, Y) \otimes C .
\end{aligned}
$$

Proof. Using Lemma 4.8, the proof will follow essentially in the same way as that of [6, Theorem 2.7].

Lemma 4.10: Let $\mathcal{D}$ be a $C$-Galois extension of $\mathcal{E}$. Then,

$$
h \otimes_{\mathcal{E}} h \cong h \otimes C
$$

as $\mathcal{D}$-corings.

Proof. We define can $: h \otimes_{\mathcal{E}} h \longrightarrow h \otimes C$ by setting $\operatorname{can}(X, Y):=\operatorname{can}_{X Y}$ for each $(X, Y) \in \operatorname{Ob}\left(\mathcal{D}^{o p} \otimes \mathcal{D}\right)$. We first verify that can is a morphism of $\mathcal{D}$ - $\mathcal{D}$ bimodules. Clearly, $\operatorname{can}(X,-)=\operatorname{can}_{X}$ which, by definition, is a morphism of left $\mathcal{D}$-modules. Therefore, it suffices to show that $\operatorname{can}(-, Y)$ is a morphism of right $\mathcal{D}$-modules, i.e., the following diagram commutes for any $g \in \operatorname{Hom}_{\mathcal{D}}\left(Z, Z^{\prime}\right)$ :

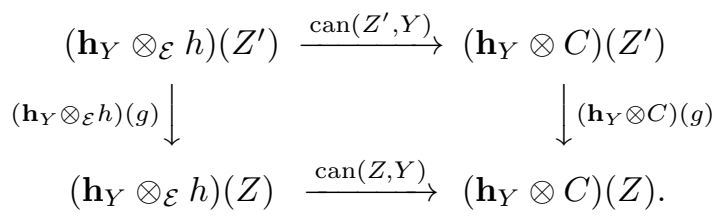

By Theorem 4.9, we know that $\mathbf{h}_{W}$ is an object in $\mathscr{M}(\psi)_{\mathcal{D}}^{C}$ for each $W \in \operatorname{Ob}(\mathcal{D})$. Thus, for any $f \in \mathbf{h}_{W}\left(Z^{\prime}\right)$, we have

$$
\begin{aligned}
(f g)_{0} \otimes(f g)_{1} & =\rho_{Z W}(f g)=\rho_{Z W}\left(\mathbf{h}_{W}(g)(f)\right) \\
& =\left(\mathbf{h}_{W} \otimes C\right)(g)\left(f_{0} \otimes f_{1}\right) \\
& =f_{0} g_{\psi} \otimes f_{1} \psi
\end{aligned}
$$


Therefore, for any $f^{\prime} \otimes f \in \mathbf{h}_{Y}(W) \otimes_{\mathcal{E}} Z^{\prime} \mathbf{h}(W)$, we obtain

$$
\begin{aligned}
\operatorname{can}(Z, Y)\left(\left(\mathbf{h}_{Y} \otimes_{\mathcal{E}} h\right)(g)\left(f^{\prime} \otimes f\right)\right) & =\operatorname{can}(Z, Y)\left(f^{\prime} \otimes f g\right) \\
& =f^{\prime} \circ(f g)_{0} \otimes(f g)_{1} \\
& =f^{\prime} f_{0} g_{\psi} \otimes f_{1}{ }^{\psi} \\
& =\left(\mathbf{h}_{Y} \otimes C\right)(g)\left(\operatorname{can}\left(Z^{\prime}, Y\right)\left(f^{\prime} \otimes f\right)\right) .
\end{aligned}
$$

It remains to verify that can is also a coalgebra morphism. First, we show that the following diagram commutes:

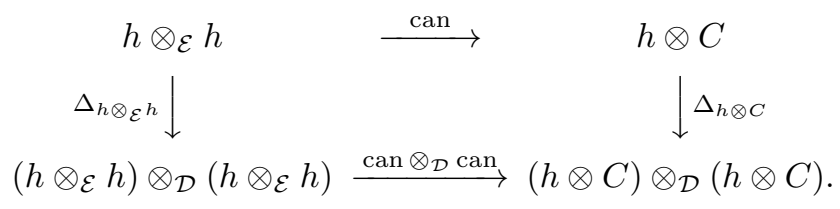

For any $(X, Y) \in \operatorname{Ob}\left(\mathcal{D}^{o p} \otimes \mathcal{D}\right)$ and $w \otimes w^{\prime} \in \mathbf{h}_{Y}(W) \otimes{ }_{X} \mathbf{h}(W)$, we have

$$
\begin{aligned}
\Delta_{h \otimes C}(X, Y) & \left(\operatorname{can}_{X Y}\left(w \otimes w^{\prime}\right)\right) \\
& =\Delta_{h \otimes C}(X, Y)\left(w w_{0}^{\prime} \otimes w_{1}^{\prime}\right)=\left(w w_{0}^{\prime} \otimes w_{11}^{\prime}\right) \otimes_{\mathcal{D}}\left(\operatorname{id}_{X} \otimes w_{12}^{\prime}\right) \\
& =\left(w w_{00}^{\prime} \otimes w_{01}^{\prime}\right) \otimes_{\mathcal{D}}\left(\operatorname{id}_{X} \otimes w_{1}^{\prime}\right) \\
& =\left({ }_{X} \mathbf{h} \otimes C\right)(w)\left(\rho_{X W}\left(w_{0}^{\prime}\right)\right) \otimes_{\mathcal{D}}\left(\operatorname{id}_{X} \otimes w_{1}^{\prime}\right) \\
& \left.=\left({ }_{X} \mathbf{h} \otimes C\right)(w)\left(\mathbf{h}_{W} \otimes C\right)\left(w_{0}^{\prime}\right)\left(\rho_{W W}\left(\operatorname{id}_{W}\right)\right)\right) \otimes_{\mathcal{D}}\left(\operatorname{id}_{X} \otimes w_{1}^{\prime}\right) \\
& =\left(\mathbf{h}_{Y} \otimes C\right)\left(w_{0}^{\prime}\right)\left(\left({ }_{W} \mathbf{h} \otimes C\right)(w)\left(\rho_{W W}\left(\operatorname{id}_{W}\right)\right)\right) \otimes_{\mathcal{D}}\left(\operatorname{id}_{X} \otimes w_{1}^{\prime}\right) \\
& =\left(\left({ }_{W} \mathbf{h} \otimes C\right)(w)\left(\rho_{W W}\left(\operatorname{id}_{W}\right)\right)\right) \cdot w_{0}^{\prime} \otimes_{\mathcal{D}}\left(\operatorname{id}_{X} \otimes w_{1}^{\prime}\right) \\
& =\left(\left({ }_{W} \mathbf{h} \otimes C\right)(w)\left(\rho_{W W}\left(\operatorname{id}_{W}\right)\right)\right) \otimes_{\mathcal{D}}\left(w_{0}^{\prime} \otimes w_{1}^{\prime}\right) \\
& =\left(w \circ \operatorname{id}_{W 0} \otimes \operatorname{id}_{W}\right) \otimes_{\mathcal{D}}\left(w_{0}^{\prime} \otimes w_{1}^{\prime}\right) \\
& =\operatorname{can}_{W Y}\left(w \otimes \operatorname{id}_{W}\right) \otimes_{\mathcal{D}} \operatorname{can}_{X W}\left(\operatorname{id}_{W} \otimes w^{\prime}\right) .
\end{aligned}
$$

It may be verified easily that can is compatible with counits. Since can is a morphism in the category of $\mathcal{D}$ - $\mathcal{D}$-bimodules and $\operatorname{can}(X, Y)=\operatorname{can}_{X Y}$ is an isomorphism for each $(X, Y) \in \mathrm{Ob}\left(\mathcal{D}^{o p} \otimes \mathcal{D}\right)$, it follows that can is an isomorphism with inverse given by

$$
\operatorname{can}^{-1}(X, Y):=\operatorname{can}_{X Y}^{-1} .
$$

This proves the result. 
Definition 4.11: Let $\mathcal{D}$ be a small $K$-linear category such that $\operatorname{Hom}_{\mathcal{D}}(X, Y)$ is a right $C$-comodule for every $X, Y \in \mathrm{Ob}(\mathcal{D})$. Let $\Phi_{X Y}: C \longrightarrow \operatorname{Hom}_{\mathcal{D}}(X, Y)$ and $\Phi_{Y Z}: C \longrightarrow \operatorname{Hom}_{\mathcal{D}}(Y, Z)$ be two $C$-comodule maps. Then, their convolution product is given by

$$
\Phi_{Y Z} * \Phi_{X Y}: C \longrightarrow \operatorname{Hom}_{\mathcal{D}}(X, Z), \quad c \mapsto \Phi_{Y Z}\left(c_{1}\right) \circ \Phi_{X Y}\left(c_{2}\right) .
$$

A collection of right $C$-comodule maps

$$
\Phi=\left\{\Phi_{X Y}: C \longrightarrow \operatorname{Hom}_{\mathcal{D}}(X, Y)\right\}_{X, Y \in \mathrm{Ob}(\mathcal{D})}
$$

is said to be convolution invertible if there exists a collection

$$
\Phi^{\prime}=\left\{\Phi_{X Y}^{\prime}: C \longrightarrow \operatorname{Hom}_{\mathcal{D}}(X, Y)\right\}_{X, Y \in \mathrm{Ob}(\mathcal{D})}
$$

of $C$-comodule maps such that

$$
\left(\Phi_{X Y} * \Phi_{Y X}^{\prime}\right)(c)=\varepsilon_{C}(c) \cdot \operatorname{id}_{Y}=\left(\Phi_{X Y}^{\prime} * \Phi_{Y X}\right)(c)
$$

for every $c \in C$.

Theorem 4.12: Let $C$ be a $K$-coalgebra and $\mathcal{D}$ be a small $K$-linear category such that $\operatorname{Hom}_{\mathcal{D}}(X, Y)$ has a right $C$-comodule structure $\rho_{X Y}$ for every $X, Y \in \operatorname{Ob}(\mathcal{D})$. Let $\mathcal{E}$ be the subcategory of $C$-coinvariants of $\mathcal{D}$. If there exists a convolution invertible collection $\Phi=\left\{\Phi_{X Y}: C \longrightarrow \operatorname{Hom}_{\mathcal{D}}(X, Y)\right\}_{X, Y \in \mathrm{Ob}(\mathcal{D})}$ of right $C$-comodule maps, then the following are equivalent:

(i) $\mathcal{D}$ is a $C$-Galois extension of $\mathcal{E}$.

(ii) There exists a right-right entwining structure $(\mathcal{D}, C, \psi)$ such that $\mathbf{h}_{Y}$ is an object in $\mathscr{M}(\psi)_{\mathcal{D}}^{C}$ for every $Y \in \operatorname{Ob}(\mathcal{D})$ with its canonical $\mathcal{D}$-module structure and right $C$-coactions $\left\{\rho_{X Y}\right\}_{X \in \mathrm{Ob}(\mathcal{D})}$.

(iii) For any $f \in \operatorname{Hom}_{\mathcal{D}}(X, Y)$, the morphism $f_{0} \circ \Phi_{Z X}^{\prime}\left(f_{1}\right) \in \operatorname{Hom}_{\mathcal{E}}(Z, Y)$ for every $Z \in \mathrm{Ob}(\mathcal{D})$, where $\Phi^{\prime}$ is the convolution inverse of $\Phi$.

Proof. By Theorem 4.9 , we have (i) $\Rightarrow$ (ii). To prove (ii) $\Rightarrow($ iii), we will use the equality

$$
\left({ }_{X} \mathbf{h} \otimes C\right)\left(\Phi_{X Y}^{\prime}(c)\right)\left(\rho_{X X}\left(\mathrm{id}_{X}\right)\right)=\psi_{X Y}\left(c_{1} \otimes \Phi_{X Y}^{\prime}\left(c_{2}\right)\right)
$$

for any $c \in C$. We first give a proof of this. Since $\mathbf{h}_{Y} \in \mathscr{M}(\psi)_{\mathcal{D}}^{C}$, we have

$$
\begin{aligned}
\rho_{X Y}(f) & =\rho_{X Y}\left(\mathbf{h}_{Y}(f)\left(\operatorname{id}_{Y}\right)\right) \\
& =\mathbf{h}_{Y}\left(f_{\psi}\right)\left(\operatorname{id}_{Y_{0}}\right) \otimes \operatorname{id}_{Y 1} \psi \\
& =\operatorname{id}_{Y_{0}} f_{\psi} \otimes \operatorname{id}_{Y 1} \psi=\left({ }_{X} \mathbf{h} \otimes C\right)\left(\operatorname{id}_{Y_{0}}\right)\left(\psi_{X Y}\left(\operatorname{id}_{Y 1} \otimes f\right)\right)
\end{aligned}
$$


for any $f \in \operatorname{Hom}_{\mathcal{D}}(X, Y)$. Also, for any $c \in C$, we have

$$
\begin{aligned}
& \left({ }_{X} \mathbf{h} \otimes C\right)\left(\operatorname{id}_{X_{0}}\right)\left(\psi_{X X}\left(\operatorname{id}_{X_{1}} \otimes \Phi_{Y X}\left(c_{1}\right) \Phi_{X Y}^{\prime}\left(c_{2}\right)\right)\right) \\
& \quad=\left({ }_{X} \mathbf{h} \otimes C\right)\left(\operatorname{id}_{X_{0}}\right)\left(\psi_{X X}\left(\operatorname{id}_{X 1} \otimes \varepsilon_{C}(c) \operatorname{id}_{X}\right)\right) \\
& \quad=\varepsilon_{C}(c) \operatorname{id}_{X_{0}} \otimes \operatorname{id}_{X 1} .
\end{aligned}
$$

Now, using (4.14), we have

$$
\begin{aligned}
& \left({ }_{X} \mathbf{h} \otimes C\right)\left(\Phi_{X Y}^{\prime}(c)\right)\left(\rho_{X X}\left(\operatorname{id}_{X}\right)\right) \\
& =\left({ }_{X} \mathbf{h} \otimes C\right)\left(\Phi_{X Y}^{\prime}\left(c_{1}\right)\right)\left(\varepsilon_{C}\left(c_{2}\right) \mathrm{id}_{X_{0}} \otimes \operatorname{id}_{X_{1}}\right) \\
& =\left({ }_{X} \mathbf{h} \otimes C\right)\left(\Phi_{X Y}^{\prime}\left(c_{1}\right)\right)\left(\left({ }_{X} \mathbf{h} \otimes C\right)\left(\operatorname{id}_{X_{0}}\right)\left(\psi_{X X}\left(\operatorname{id}_{X 1} \otimes \Phi_{Y X}\left(c_{2}\right) \Phi_{X Y}^{\prime}\left(c_{3}\right)\right)\right)\right) \\
& \text { (using (4.14)) } \\
& =\left({ }_{X} \mathbf{h} \otimes C\right)\left(\Phi_{X Y}^{\prime}\left(c_{1}\right)\right)\left(\left({ }_{X} \mathbf{h} \otimes C\right)\left(\operatorname{id}_{X 0}\right)\left(\left(\Phi_{Y X}\left(c_{2}\right)\right)_{\psi}\left(\Phi_{X Y}^{\prime}\left(c_{3}\right)\right)_{\psi} \otimes \operatorname{id}_{X 1}{ }^{\psi}{ }^{\psi}\right)\right) \\
& \text { (using (2.1)) } \\
& =\left({ }_{X} \mathbf{h} \otimes C\right)\left(\Phi_{X Y}^{\prime}\left(c_{1}\right)\right)\left(\operatorname{id}_{X 0} \circ\left(\Phi_{Y X}\left(c_{2}\right)\right)_{\psi}\left(\Phi_{X Y}^{\prime}\left(c_{3}\right)\right)_{\psi} \otimes \operatorname{id}_{X 1}{ }^{\psi}{ }^{\psi}\right) \\
& =\left({ }_{X} \mathbf{h} \otimes C\right)\left(\Phi_{X Y}^{\prime}\left(c_{1}\right)\right)\left(\left(\mathbf{h}_{X} \otimes C\right)\left(\Phi_{X Y}^{\prime}\left(c_{3}\right)\right)\left(\operatorname{id}_{X_{0}} \circ\left(\Phi_{Y X}\left(c_{2}\right)\right)_{\psi} \otimes \operatorname{id}_{X 1}{ }^{\psi}\right)\right) \\
& =\left({ }_{X} \mathbf{h} \otimes C\right)\left(\Phi_{X Y}^{\prime}\left(c_{1}\right)\right) \\
& \times\left(\left(\mathbf{h}_{X} \otimes C\right)\left(\Phi_{X Y}^{\prime}\left(c_{3}\right)\right)\left(\left({ }_{Y} \mathbf{h} \otimes C\right)\left(\operatorname{id}_{X_{0}}\right)\left(\psi_{Y X}\left(\operatorname{id}_{X_{1}} \otimes \Phi_{Y X}\left(c_{2}\right)\right)\right)\right)\right) \\
& =\left({ }_{X} \mathbf{h} \otimes C\right)\left(\Phi_{X Y}^{\prime}\left(c_{1}\right)\right)\left(\left(\mathbf{h}_{X} \otimes C\right)\left(\Phi_{X Y}^{\prime}\left(c_{3}\right)\right)\left(\rho_{Y X}\left(\Phi_{Y X}\left(c_{2}\right)\right)\right)\right) \\
& =\left({ }_{X} \mathbf{h} \otimes C\right)\left(\Phi_{X Y}^{\prime}\left(c_{1}\right)\right)\left(\left(\mathbf{h}_{X} \otimes C\right)\left(\Phi_{X Y}^{\prime}\left(c_{4}\right)\right)\left(\Phi_{Y X}\left(c_{2}\right) \otimes c_{3}\right)\right)
\end{aligned}
$$

(since $\Phi_{Y X}$ is $C$-colinear)

$$
=\Phi_{X Y}^{\prime}\left(c_{1}\right) \Phi_{Y X}\left(c_{2}\right)\left(\Phi_{X Y}^{\prime}\left(c_{4}\right)\right)_{\psi} \otimes c_{3}{ }^{\psi}
$$$$
=\varepsilon_{C}\left(c_{1}\right) \operatorname{id}_{Y}\left(\Phi_{X Y}^{\prime}\left(c_{3}\right)\right)_{\psi} \otimes c_{2}{ }^{\psi}
$$$$
=\left(\mathbf{h}_{Y} \otimes C\right)\left(\Phi_{X Y}^{\prime}\left(c_{3}\right)\right)\left(\varepsilon_{C}\left(c_{1}\right) \mathrm{id}_{Y} \otimes c_{2}\right)
$$$$
=\left(\mathbf{h}_{Y} \otimes C\right)\left(\Phi_{X Y}^{\prime}\left(c_{2}\right)\right)\left(\mathrm{id}_{Y} \otimes c_{1}\right)
$$$$
=\left(\Phi_{X Y}^{\prime}\left(c_{2}\right)\right)_{\psi} \otimes c_{1}{ }^{\psi}=\psi_{X Y}\left(c_{1} \otimes \Phi_{X Y}^{\prime}\left(c_{2}\right)\right)
$$

This proves the equality (4.12).

For any $f \in \operatorname{Hom}_{\mathcal{D}}(X, Y)$, consider the morphism $f_{0} \circ \Phi_{Z X}^{\prime}\left(f_{1}\right): Z \longrightarrow Y$ in $\mathcal{D}$. Then $f_{0} \circ \Phi_{Z X}^{\prime}\left(f_{1}\right)$ induces a morphism of right $\mathcal{D}$-modules $\mathbf{h}_{Z} \longrightarrow \mathbf{h}_{Y}$ which we denote by $\tilde{f}$. We now verify that the map $\tilde{f}\left(X^{\prime}\right): \mathbf{h}_{Z}\left(X^{\prime}\right) \longrightarrow \mathbf{h}_{Y}\left(X^{\prime}\right)$ 
is right $C$-colinear for each $X^{\prime} \in \operatorname{Ob}(\mathcal{D})$. Since $\mathbf{h}_{Y}$ is an object in $\mathscr{M}(\psi)_{\mathcal{D}}^{C}$ for every $Y \in \operatorname{Ob}(\mathcal{D})$, the following diagram commutes for any $g \in \operatorname{Hom}_{\mathcal{D}}\left(X^{\prime}, Z\right)$ :

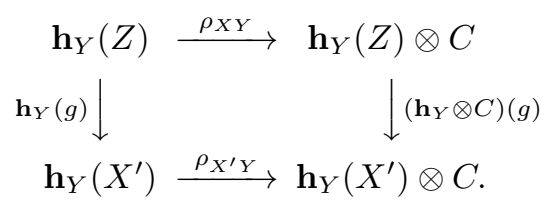

Thus, we have

$$
\begin{aligned}
\rho_{X^{\prime} Y} & \left.\tilde{f}\left(X^{\prime}\right)(g)\right) \\
& =\rho_{X^{\prime} Y}\left(f_{0} \circ \Phi_{Z X}^{\prime}\left(f_{1}\right) \circ g\right) \\
& =\rho_{X^{\prime} Y}\left(\mathbf{h}_{Y}\left(\Phi_{Z X}^{\prime}\left(f_{1}\right) \circ g\right)\left(f_{0}\right)\right) \\
& =\left(\mathbf{h}_{Y} \otimes C\right)\left(\Phi_{Z X}^{\prime}\left(f_{1}\right) \circ g\right)\left(\rho_{X Y}\left(f_{0}\right)\right) \\
& =\left(\mathbf{h}_{Y} \otimes C\right)(g)\left(\left(\mathbf{h}_{Y} \otimes C\right)\left(\Phi_{Z X}^{\prime}\left(f_{1}\right)\right)\left(f_{00} \otimes f_{01}\right)\right) \\
& =\left(\mathbf{h}_{Y} \otimes C\right)(g)\left(f_{0}\left(\Phi_{Z X}^{\prime}\left(f_{12}\right)\right)_{\psi} \otimes f_{11} \psi\right) \\
& =\left(\mathbf{h}_{Y} \otimes C\right)(g)\left(\left({ }_{Z} \mathbf{h} \otimes C\right)\left(f_{0}\right)\left(\psi_{Z X}\left(f_{11} \otimes \Phi_{Z X}^{\prime}\left(f_{12}\right)\right)\right)\right) \\
& =\left(\mathbf{h}_{Y} \otimes C\right)(g)\left((Z \mathbf{h} \otimes C)\left(f_{0}\right)(Z \mathbf{h} \otimes C)\left(\left(\Phi_{Z X}^{\prime}\right)\left(f_{1}\right)\right)\left(\rho_{Z Z}\left(\operatorname{id}_{Z}\right)\right)\right)(\text { using }(4.12)) \\
& =\left(\mathbf{h}_{Y} \otimes C\right)(g)\left(\left({ }_{Z} \mathbf{h} \otimes C\right)\left(f_{0} \circ \Phi_{Z X}^{\prime}\left(f_{1}\right)\right)\left(\rho_{Z Z}\left(\operatorname{id}_{Z}\right)\right)\right) \\
& =(h \otimes C)\left(g, f_{0} \circ \Phi_{Z X}^{\prime}\left(f_{1}\right)\right)\left(\rho_{Z Z}\left(\operatorname{id}_{Z}\right)\right) \\
& =\left(X^{\prime} \mathbf{h} \otimes C\right)\left(f_{0} \circ \Phi_{Z X}^{\prime}\left(f_{1}\right)\right)\left(\left(\mathbf{h}_{Z} \otimes C\right)(g)\left(\rho_{Z Z}\left(\operatorname{id}_{Z}\right)\right)\right) \\
& =\left(\tilde{f}\left(X^{\prime}\right) \otimes \operatorname{id}_{C}\right)\left(\left(\mathbf{h}_{Z} \otimes C\right)(g)\left(\rho_{Z Z}\left(\operatorname{id}_{Z}\right)\right)\right) \\
& =\left(\tilde{f}\left(X^{\prime}\right) \otimes \operatorname{id}_{C}\right)\left(\rho_{X^{\prime} Z}(g)\right) .
\end{aligned}
$$

Therefore, $\tilde{f} \in \operatorname{Hom}_{\text {Mod -D }}^{C}\left(\mathbf{h}_{Z}, \mathbf{h}_{Y}\right)=\operatorname{Hom}_{\mathcal{E}}(Z, Y)$.

For (iii) $\Rightarrow(\mathrm{i})$, we start by showing that

$$
\operatorname{can}_{X Y}: \mathbf{h}_{Y} \otimes_{\mathcal{E} X} \mathbf{h} \longrightarrow \operatorname{Hom}_{\mathcal{D}}(X, Y) \otimes C
$$

is an isomorphism for each $X, Y \in \mathrm{Ob}(\mathcal{D})$. We define

$$
\operatorname{can}_{X Y}^{-1}: \operatorname{Hom}_{\mathcal{D}}(X, Y) \otimes C \longrightarrow \mathbf{h}_{Y} \otimes_{\mathcal{E} X} \mathbf{h}
$$

by

$$
\operatorname{can}_{X Y}^{-1}(f \otimes c):=f \circ \Phi_{Y X}^{\prime}\left(c_{1}\right) \otimes_{\mathcal{E}} \Phi_{X Y}\left(c_{2}\right) \in \mathbf{h}_{Y} \otimes_{\mathcal{E} X} \mathbf{h}
$$


for any $f \in \operatorname{Hom}_{\mathcal{D}}(X, Y)$ and $c \in C$. Then, using the $C$-colinearity of $\Phi_{X Y}$, we have

$$
\begin{aligned}
\left(\operatorname{can}_{X Y} \circ \operatorname{can}_{X Y}^{-1}\right)(f \otimes c) & =f \circ \Phi_{Y X}^{\prime}\left(c_{1}\right) \circ\left(\Phi_{X Y}\left(c_{2}\right)\right)_{0} \otimes\left(\Phi_{X Y}\left(c_{2}\right)\right)_{1} \\
& =f \circ \Phi_{Y X}^{\prime}\left(c_{1}\right) \circ \Phi_{X Y}\left(c_{2}\right) \otimes c_{3}=f \otimes c .
\end{aligned}
$$

On the other hand, by assumption, we obtain

$$
\begin{aligned}
\left(\operatorname{can}_{X Y}^{-1} \circ \operatorname{can}_{X Y}\right)\left(g \otimes_{\mathcal{E}} g^{\prime}\right) & =g g_{0}^{\prime} \Phi_{Y X}^{\prime}\left(g_{11}^{\prime}\right) \otimes_{\mathcal{E}} \Phi_{X Y}\left(g_{12}^{\prime}\right) \\
& =g \otimes_{\mathcal{E}} g_{0}^{\prime} \Phi_{Y X}^{\prime}\left(g_{11}^{\prime}\right) \Phi_{X Y}\left(g_{12}^{\prime}\right)=g \otimes_{\mathcal{E}} g^{\prime}
\end{aligned}
$$

for any $g \otimes_{\mathcal{E}} g^{\prime} \in \mathbf{h}_{Y} \otimes_{\mathcal{E} X} \mathbf{h}$. From the definition in (4.16), it is clear that setting $\operatorname{can}_{X}^{-1}(Y):=\operatorname{can}_{X Y}^{-1}$ for each $Y \in \mathrm{Ob}(\mathcal{D})$ determines a morphism in $\mathcal{D} \mathscr{M}^{C}$ which is inverse to $\operatorname{can}_{X}$. This completes the proof.

Example 4.13: Let $H$ be a Hopf algebra over $K$. If $\mathcal{C}$ is a left $H$-module category, then the smash product category $\mathcal{C} \# H$ (see [16]) is a right co- $H$-category with the right $H$-coaction determined by

$$
f \# h \mapsto f \# h_{1} \otimes h_{2}
$$

on each $\operatorname{Hom}_{\mathcal{C} \# H}(X, Y)=\operatorname{Hom}_{\mathcal{C}}(X, Y) \otimes H$. By definition, we know that

$$
\mathrm{Ob}(\mathcal{C})=\operatorname{Ob}(\mathcal{C} \# H)
$$

It is easy to see that

$$
\operatorname{Hom}_{\mathcal{C}}(X, Y)=\operatorname{Hom}_{\mathcal{C} \# H}(X, Y)^{c o H} .
$$

We claim that $\mathcal{C} \# H$ is an $H$-Galois extension of $\mathcal{C}$. We first observe that for any $f \# h \in \operatorname{Hom}_{\mathcal{C} \# H}(Z, Y)$ and $f^{\prime} \# h^{\prime} \in \operatorname{Hom}_{\mathcal{C} \# H}(X, Z)$, we have

$$
(f \# h) \otimes_{\mathcal{C}}\left(f^{\prime} \# h^{\prime}\right)=(f \# h)\left(f^{\prime} \# 1_{H}\right) \otimes_{\mathcal{C}}\left(\operatorname{id}_{X} \# h^{\prime}\right) .
$$

Thus, $\operatorname{can}_{X Y}: \mathbf{h}_{Y} \otimes_{\mathcal{C} X} \mathbf{h} \longrightarrow \operatorname{Hom}_{\mathcal{C} \# H}(X, Y) \otimes H$ has the following form:

$$
\operatorname{can}_{X Y}\left((f \# h) \otimes_{\mathcal{C}}\left(f^{\prime} \# h^{\prime}\right)\right)=(f \# h)\left(f^{\prime} \# 1_{H}\right)\left(\operatorname{id}_{X} \# h_{1}^{\prime}\right) \otimes h_{2}^{\prime}
$$

for each $X, Y \in \mathrm{Ob}(\mathcal{C} \# H)$. Then, it may be verified that for each $X, Y \in \mathrm{Ob}(\mathcal{C} \# H)$, $\operatorname{can}_{X Y}$ is an isomorphism with inverse $\operatorname{can}_{X Y}^{-1}: \operatorname{Hom}_{\mathcal{C} \# H}(X, Y) \otimes H \longrightarrow \mathbf{h}_{Y} \otimes_{\mathcal{C} X} \mathbf{h}$ determined by

$$
\operatorname{can}_{X Y}^{-1}\left((g \# k) \otimes k^{\prime}\right):=(g \# k)\left(\operatorname{id}_{X} \# S\left(k_{1}^{\prime}\right)\right) \otimes_{\mathcal{C}}\left(\operatorname{id}_{X} \# k_{2}^{\prime}\right) .
$$


Proposition 4.14: Let $\mathcal{D}$ be a $C$-Galois extension of $\mathcal{E}$. If there exists a convolution invertible collection

$$
\Phi=\left\{\Phi_{X Y}: C \longrightarrow \operatorname{Hom}_{\mathcal{D}}(X, Y)\right\}_{X, Y \in \mathrm{Ob}(\mathcal{D})}
$$

of right $C$-comodule maps, then

$$
\operatorname{Hom}_{\mathcal{D}}(X,-) \cong \operatorname{Hom}_{\mathcal{E}}(X,-) \otimes C \in \mathcal{E}^{C}
$$

for each $X \in \mathrm{Ob}(\mathcal{E})=\mathrm{Ob}(\mathcal{D})$.

Proof. Let $\Phi^{\prime}$ be the convolution inverse of $\Phi$. Given $f \in \operatorname{Hom}_{\mathcal{D}}(X, Y)$, it follows from Theorem 4.12 that $f_{0} \circ \Phi_{Z X}^{\prime}\left(f_{1}\right) \in \operatorname{Hom}_{\mathcal{E}}(Z, Y)$ for every $Z \in \operatorname{Ob}(\mathcal{D})$. We define

$$
\eta: \operatorname{Hom}_{\mathcal{D}}(X,-) \longrightarrow \operatorname{Hom}_{\mathcal{E}}(X,-) \otimes C, \quad \eta(Y)(f):=f_{0} \circ \Phi_{X X}^{\prime}\left(f_{1}\right) \otimes f_{2} .
$$

Using Definition 4.5, we see that $\rho_{X Y^{\prime}}(g f)=g f_{0} \otimes f_{1}$ for any $g \in \operatorname{Hom}_{\mathcal{E}}\left(Y, Y^{\prime}\right)$. Hence, we have

$$
\begin{aligned}
(g f)_{0} \otimes(g f)_{1} \otimes(g f)_{2} & =\left(\mathrm{id} \otimes \Delta_{C}\right)\left((g f)_{0} \otimes(g f)_{1}\right) \\
& =\left(\rho_{X Y^{\prime}} \otimes \mathrm{id}_{C}\right)\left(g f_{0} \otimes f_{1}\right) \\
& =g f_{0} \otimes f_{1} \otimes f_{2} .
\end{aligned}
$$

Using (4.17), it may be easily seen that $\eta$ is a morphism of left $\mathcal{E}$-modules. Using the coassociativity of the $C$-coactions $\left\{\rho_{X Y}\right\}_{X, Y \in \mathrm{Ob}(\mathcal{D})}$, it is also clear that $\eta$ is objectwise $C$-colinear. Therefore, $\eta$ is a morphism in $\mathcal{E} \mathscr{M}^{C}$.

Conversely, we define $\zeta: \operatorname{Hom}_{\mathcal{E}}(X,-) \otimes C \longrightarrow \operatorname{Hom}_{\mathcal{D}}(X,-)$ given by

$$
\zeta(Y)\left(f^{\prime} \otimes c\right):=f^{\prime} \circ \Phi_{X X}(c) \text { for } Y \in \mathrm{Ob}(\mathcal{E}) .
$$

It is immediate that $\zeta$ is a morphism of left $\mathcal{E}$-modules. Moreover,

$$
\begin{aligned}
\rho_{X Y}\left(f^{\prime} \circ \Phi_{X X}(c)\right) & =\left(f^{\prime} \circ \Phi_{X X}(c)\right)_{0} \otimes\left(f^{\prime} \circ \Phi_{X X}(c)\right)_{1} \\
& =f^{\prime} \circ\left(\Phi_{X X}(c)\right)_{0} \otimes\left(\Phi_{X X}(c)\right)_{1} \\
& =f^{\prime} \circ \Phi_{X X}\left(c_{1}\right) \otimes c_{2}
\end{aligned}
$$

where the last equality follows from the fact that $\Phi_{X X}$ is $C$-colinear. It follows that $\zeta(Y)$ is $C$-colinear for each $Y \in \operatorname{Ob}(\mathcal{E})$ and hence $\zeta$ is a morphism in $\mathcal{E} \mathscr{M}^{C}$. It may be verified that $\zeta$ is the inverse of $\eta$. 
Definition 4.15: Let $\mathcal{D}$ be a small $K$-linear category and $\mathcal{E}$ be a $K$-subcategory. Let $\left(\mathscr{C}, \Delta_{\mathscr{C}}, \varepsilon_{\mathscr{C}}\right)$ be a $\mathcal{D}$-coring. Then, a collection

$$
G(\mathscr{C}, \mathcal{E})=\left\{s_{X} \in \mathscr{C}(X, X)\right\}_{X \in \operatorname{Ob}(\mathcal{E})}
$$

is said to be group-like for $\mathscr{C}$ with respect to $\mathcal{E}$ if:

(i) $\Delta_{\mathscr{C}}(X, X)\left(s_{X}\right)=s_{X} \otimes s_{X}$ and $\varepsilon_{\mathscr{C}}\left(s_{X}\right)=\mathrm{id}{ }_{X}$ for any $X \in \mathrm{Ob}(\mathcal{E})$,

(ii) For any $f \in \operatorname{Hom}_{\mathcal{E}}(X, Y)$, we have

$$
f \cdot s_{X}=\mathscr{C}(-, f)(X)\left(s_{X}\right)=\mathscr{C}(f,-)(Y)\left(s_{Y}\right)=s_{Y} \cdot f .
$$

Example 4.16: (i) If $\mathcal{E}$ is a subcategory of $\mathcal{D}$, then the collection

$$
\left\{\operatorname{id}_{X} \otimes \operatorname{id}_{X} \in \mathbf{h}_{X} \otimes_{\mathcal{E} X} \mathbf{h}\right\}_{X \in \mathrm{Ob}(\mathcal{E})}
$$

is group-like for $h \otimes_{\mathcal{E}} h$ with respect to $\mathcal{E}$.

(ii) Let $\mathcal{D}$ be a $C$-Galois extension of $\mathcal{E}$. Then $h \otimes C$ is a $\mathcal{D}$-coring (by Theorem 4.9 and Lemma 4.2) and the collection

$$
\left\{\operatorname{id}_{X_{0}} \otimes \operatorname{id}_{X_{1}} \in \operatorname{Hom}_{\mathcal{D}}(X, X) \otimes C\right\}_{X \in \mathrm{Ob}(\mathcal{E})}
$$

is group-like for $h \otimes C$ with respect to $\mathcal{E}$. Since $\mathbf{h}_{Y} \in \mathscr{M}(\psi)_{\mathcal{D}}^{C}$ for each $Y \in \mathrm{Ob}(\mathcal{D})$, we have

$$
\begin{aligned}
\rho_{X Y}(f) & =\rho_{X Y}\left(\mathbf{h}_{Y}(f)\left(\operatorname{id}_{Y}\right)\right)=\mathbf{h}_{Y}\left(f_{\psi}\right)\left(\operatorname{id}_{Y_{0}}\right) \otimes \operatorname{id}_{Y 1}{ }^{\psi} \\
& =\operatorname{id}_{Y_{0}} f_{\psi} \otimes \operatorname{id}_{Y 1} \psi \\
& =\left(\operatorname{id}_{Y_{0}} \otimes \operatorname{id}_{Y 1}\right) \cdot f
\end{aligned}
$$

for any $f \in \operatorname{Hom}_{\mathcal{D}}(X, Y)$. But, if $f \in \operatorname{Hom}_{\mathcal{E}}(X, Y)$, then we also have $\rho_{X Y}(f)=\rho_{X Y}\left(f \circ \operatorname{id}_{X}\right)=f \cdot \rho_{X X}\left(\operatorname{id}_{X}\right)=f \circ \operatorname{id}_{X 0} \otimes \operatorname{id}_{X 1}=f \cdot\left(\operatorname{id}_{X_{0}} \otimes \operatorname{id}_{X_{1}}\right)$.

Proposition 4.17: Let $\mathcal{E} \subseteq \mathcal{D}$ be a subcategory and $\mathscr{C}$ be a $\mathcal{D}$-coring. Let $\left\{s_{X}\right\}_{X \in \mathrm{Ob}(\mathcal{E})}$ be a group-like collection for $\mathscr{C}$ with respect to $\mathcal{E}$. For a right $\mathscr{C}$-comodule $\left(\mathcal{N}, \rho_{\mathcal{N}}\right)$, the $\mathcal{E}$-submodule $\mathcal{N}^{\text {co } \mathscr{C}}: \mathcal{E}^{o p} \longrightarrow$ Vect $_{K}$ of coinvariants of $\mathcal{N}$ is given by

$$
\begin{aligned}
& \mathcal{N}^{\operatorname{co} \mathscr{C}}(X):=\left\{n \in \mathcal{N}(X) \mid \rho_{\mathcal{N}}(X)(n)=n \otimes s_{X}\right\}, \\
& \mathcal{N}^{\operatorname{co} \mathscr{C}}(f)\left(n^{\prime}\right):=\mathcal{N}(f)\left(n^{\prime}\right)
\end{aligned}
$$

for any $X \in \operatorname{Ob}(\mathcal{E}), f \in \operatorname{Hom}_{\mathcal{E}}(X, Y)$ and $n^{\prime} \in \mathcal{N}^{\operatorname{co} \mathscr{C}}(Y)$. 
Proof. We will show that for any $f \in \operatorname{Hom}_{\mathcal{E}}(X, Y)$, the morphism

$$
\mathcal{N}^{\operatorname{coC}^{\mathscr{C}}}(f): \mathcal{N}^{\operatorname{co} \mathscr{C}}(Y) \longrightarrow \mathcal{N}^{\operatorname{co} \mathscr{C}}(X)
$$

is well-defined. Since $\rho_{\mathcal{N}}: \mathcal{N} \longrightarrow \mathcal{N} \otimes_{\mathcal{D}} \mathscr{C}$ is a morphism of right $\mathcal{D}$-modules, we have the following commutative diagram:

$$
\begin{array}{lll}
\mathcal{N}(Y) \stackrel{\rho_{\mathcal{N}}(Y)}{\longrightarrow} & \mathcal{N} \otimes_{\mathcal{D}} \mathscr{C}(Y,-) \\
\mathcal{N}(f) \downarrow & \\
\mathcal{N}(X) \stackrel{\rho_{\mathcal{N}}(X)}{\longrightarrow} \mathcal{N} \otimes_{\mathcal{D}} \mathscr{C}(X,-) .
\end{array}
$$

Let $n^{\prime} \in \mathcal{N}^{\operatorname{co} \mathscr{C}}(Y)$ so that $\rho_{\mathcal{N}}(Y)\left(n^{\prime}\right)=n^{\prime} \otimes s_{Y}$. Since $f \in \operatorname{Hom}_{\mathcal{E}}(X, Y)$, using (4.18) we have

$$
\begin{aligned}
\rho_{\mathcal{N}}(X)\left(\mathcal{N}(f)\left(n^{\prime}\right)\right) & =\left(\operatorname{id}_{\mathcal{N}} \otimes_{C}(f,-)\right)\left(n^{\prime} \otimes_{\mathcal{D}} s_{Y}\right) \\
& =n^{\prime} \otimes_{\mathcal{D}} s_{Y} \cdot f=n^{\prime} \otimes_{\mathcal{D}} f \cdot s_{X}=\mathcal{N}(f)\left(n^{\prime}\right) \otimes_{\mathcal{D}} s_{X} .
\end{aligned}
$$

This shows that $\mathcal{N}(f)\left(n^{\prime}\right)=\mathcal{N}^{\operatorname{co} \mathscr{C}}(f)\left(n^{\prime}\right) \in \mathcal{N}^{\operatorname{co} \mathscr{C}}(X)$. The result follows.

The next result shows that in the case of a $C$-Galois extension $\mathcal{E} \subseteq \mathcal{D}$, we recover the notion of coinvariants as in Definition 4.5.

Lemma 4.18: Let $\mathcal{D}$ be a $C$-Galois extension of $\mathcal{E}$. Consider the collection

$$
\left\{\operatorname{id}_{X 0} \otimes \operatorname{id}_{X 1} \in \operatorname{Hom}_{\mathcal{D}}(X, X) \otimes C\right\}_{X \in \mathrm{Ob}(\mathcal{D})}
$$

which is group-like for $h \otimes C$ with respect to $\mathcal{E}$. Then,

$$
\left(\operatorname{Hom}_{\mathcal{D}}(-, Y)\right)^{c o(h \otimes C)}(X)=\operatorname{Hom}_{\mathcal{E}}(X, Y)
$$

for any $X, Y \in \mathrm{Ob}(\mathcal{D})=\mathrm{Ob}(\mathcal{E})$.

Proof. Since $\mathcal{D}$ is a $C$-Galois extension of $\mathcal{E}$, we know that there is a canonical entwining $(\mathcal{D}, C, \psi)$ such that $\mathbf{h}_{Y} \in \mathscr{M}(\psi)_{\mathcal{D}}^{C}$. Using Proposition $4.3, \mathbf{h}_{Y}$ may be treated as an object of Comod- $(h \otimes C)$. Let $g \in\left(\operatorname{Hom}_{\mathcal{D}}(-, Y)\right)^{c o(h \otimes C)}(X)$. Then, $\rho_{X Y}(g)=g \circ \operatorname{id}_{X_{0}} \otimes \operatorname{id}_{X_{1}}$. Using the fact that $\mathbf{h}_{Y} \in \mathscr{M}(\psi)_{\mathcal{D}}^{C}$ we have

$$
\begin{aligned}
\rho_{Z Y}(g f) & =\left(\mathbf{h}_{Y} \otimes C\right)(f)\left(\rho_{X Y}(g)\right)=\left(\mathbf{h}_{Y} \otimes C\right)(f)\left(g \circ \operatorname{id}_{X 0} \otimes \operatorname{id}_{X 1}\right) \\
& =g \circ \operatorname{id}_{X 0} \circ f_{\psi} \otimes \operatorname{id}_{X 1}{ }^{\psi}=\left({ }_{Z} \mathbf{h} \otimes C\right)(g)\left(\operatorname{id}_{X_{0}} \circ f_{\psi} \otimes \operatorname{id}_{X 1}{ }^{\psi}\right) \\
& =\left({ }_{Z} \mathbf{h} \otimes C\right)(g) \rho_{Z X}(f)
\end{aligned}
$$

for any $f \in \operatorname{Hom}_{\mathcal{D}}(Z, X)$. Therefore, $g \in \operatorname{Hom}_{\mathcal{E}}(X, Y)$. The converse follows directly using the Definition 4.5. 
Lemma 4.19: Let $\mathcal{D}$ be a $C$-Galois extension of $\mathcal{E}$ and let $(\mathcal{D}, C, \psi)$ be the canonical entwining structure associated to it. We denote by

$$
\rho_{X Y}: \operatorname{Hom}_{\mathcal{D}}(X, Y) \longrightarrow \operatorname{Hom}_{\mathcal{D}}(X, Y) \otimes C
$$

the right $C$-comodule structure maps. Then, for any $\mathcal{M} \in \operatorname{Mod}-\mathcal{E}$, we may obtain an object $\mathcal{M} \otimes_{\mathcal{E}} h \in \mathscr{M}(\psi)_{\mathcal{D}}^{C}$ by setting

$$
\left(\mathcal{M} \otimes_{\mathcal{E}} h\right)(Y):=\mathcal{M} \otimes_{\mathcal{E} Y} \mathbf{h}, \quad\left(\mathcal{M} \otimes_{\mathcal{E}} h\right)(f)(m \otimes g):=m \otimes g f
$$

for $f \in \operatorname{Hom}_{\mathcal{D}}(X, Y)$ and $m \otimes g \in \mathcal{M}(Z) \otimes_{Y} \mathbf{h}(Z)$. In fact, this determines a functor from $\operatorname{Mod}-\mathcal{E}$ to $\mathscr{M}(\psi)_{\mathcal{D}}^{C}$.

Proof. Clearly, $\mathcal{M} \otimes_{\mathcal{E}} h \in \operatorname{Mod}-\mathcal{D}$. For each $Y \in \mathrm{Ob}(\mathcal{D})$, it may be verified that $\mathcal{M} \otimes_{\mathcal{E} Y} \mathbf{h}$ has a right $C$-comodule structure given by

$$
\mathcal{M} \otimes_{\mathcal{E} Y} \mathbf{h} \stackrel{\operatorname{id} \otimes \rho}{\longrightarrow} \mathcal{M} \otimes_{\mathcal{E} Y} \mathbf{h} \otimes C, \quad m \otimes g \mapsto m \otimes \rho_{Y Z}(g),
$$

for any $g \in \operatorname{Hom}_{\mathcal{D}}(Y, Z)$ and $m \in \mathcal{M}(Z)$. By Theorem 4.9, $\mathbf{h}_{Z}$ is an object in $\mathscr{M}(\psi)_{\mathcal{D}}^{C}$ for every $Z \in \mathrm{Ob}(\mathcal{D})$ with its canonical $\mathcal{D}$-module structure and right $C$-coactions $\left\{\rho_{X Z}\right\}_{X \in \mathrm{Ob}(\mathcal{D})}$. Therefore, we have

$$
\left.\rho_{X Z}\left(\mathbf{h}_{Z}(f)(g)\right)\right)=(g f)_{0} \otimes(g f)_{1}=g_{0} f_{\psi} \otimes g_{1}^{\psi}
$$

for any $f \in \operatorname{Hom}_{\mathcal{D}}(X, Y)$. Consequently, we have

(4.19) $\left(\mathrm{id} \otimes \rho_{X Z}\right)\left(\left(\mathcal{M} \otimes_{\mathcal{E}} h\right)(f)(m \otimes g)\right)=m \otimes(g f)_{0} \otimes(g f)_{1}=m \otimes g_{0} f_{\psi} \otimes g_{1}^{\psi}$.

This shows that $\mathcal{M} \otimes_{\mathcal{E}} h \in \mathscr{M}(\psi)_{\mathcal{D}}^{C}$.

Lemma 4.20: Let $\mathcal{D}$ be a $C$-Galois extension of $\mathcal{E}$. If there exists a convolution invertible collection $\Phi=\left\{\Phi_{X Y}: C \longrightarrow \operatorname{Hom}_{\mathcal{D}}(X, Y)\right\}_{X, Y \in \mathrm{Ob}(\mathcal{D})}$ of right $C$ comodule maps, then:

(i) $\operatorname{Hom}_{\mathcal{D}}(X,-)$ is flat as a left $\mathcal{E}$-module.

(ii) $\bigoplus_{X \in \mathrm{Ob}(\mathcal{D})} \operatorname{Hom}_{\mathcal{D}}(X,-)$ is faithfully flat as a left $\mathcal{E}$-module.

(iii) For any $\mathcal{M} \in \operatorname{Mod}-\mathcal{E}$, there is a monomorphism $\mathcal{M} \hookrightarrow \mathcal{M} \otimes_{\mathcal{E}} h$ in Mod $-\mathcal{E}$ given by $m \mapsto m \otimes \operatorname{id}_{X}$ for any $m \in \mathcal{M}(X)$.

Proof. (i) Let $i: \mathcal{M}_{1} \hookrightarrow \mathcal{M}_{2}$ be a monomorphism of right $\mathcal{E}$-modules. By Proposition 4.14, it follows that the induced map

$$
\mathcal{M}_{1} \otimes_{\mathcal{E}} \operatorname{Hom}_{\mathcal{D}}(X,-) \longrightarrow \mathcal{M}_{2} \otimes_{\mathcal{E}} \operatorname{Hom}_{\mathcal{D}}(X,-)
$$


coincides with the map

$$
\mathcal{M}_{1}(X) \otimes C \stackrel{i(X) \otimes \mathrm{id}_{C}}{\longrightarrow} \mathcal{M}_{2}(X) \otimes C
$$

for each $X \in \mathrm{Ob}(\mathcal{E})=\mathrm{Ob}(\mathcal{D})$. Since $i(X) \otimes \mathrm{id}_{C}$ is clearly a monomorphism, it follows that $\operatorname{Hom}_{\mathcal{D}}(X,-)$ is flat as a left $\mathcal{E}$-module.

(ii) This is clear from the fact that

$$
\mathcal{M}(X) \otimes C=\mathcal{M} \otimes_{\mathcal{E}} \operatorname{Hom}_{\mathcal{D}}(X,-)=0 \Rightarrow \mathcal{M}(X)=0 .
$$

(iii) Since $\bigoplus_{Y \in \operatorname{Ob}(\mathcal{D})} \operatorname{Hom}_{\mathcal{D}}(Y,-)$ is faithfully flat as a left $\mathcal{E}$-module, it is enough to prove that for each $Y \in \mathrm{Ob}(\mathcal{D})$, we have a monomorphism

$$
\begin{aligned}
\mathcal{M} \otimes_{\mathcal{E}} \operatorname{Hom}_{\mathcal{D}}(Y,-) & \longrightarrow \mathcal{M} \otimes_{\mathcal{E}} h \otimes_{\mathcal{E}} \operatorname{Hom}_{\mathcal{D}}(Y,-), \\
\mathcal{M}(X) \otimes_{Y} \mathbf{h}(X) \ni m \otimes f & \mapsto m \otimes \operatorname{id}_{X} \otimes f .
\end{aligned}
$$

This is true because the morphism in (4.20) has a section

$$
\begin{gathered}
\mathcal{M} \otimes_{\mathcal{E}} h \otimes_{\mathcal{E}} \operatorname{Hom}_{\mathcal{D}}(Y,-) \longrightarrow \mathcal{M} \otimes_{\mathcal{E}} \operatorname{Hom}_{\mathcal{D}}(Y,-), \\
m^{\prime} \otimes g^{\prime} \otimes f^{\prime} \mapsto m \otimes g^{\prime} f^{\prime}
\end{gathered}
$$

for any $m^{\prime} \in \mathcal{M}(Z)$ and $g^{\prime} \otimes f^{\prime} \in{ }_{X} \mathbf{h}(Z) \otimes_{Y} \mathbf{h}(X)$.

Theorem 4.21: Let $\mathcal{D}$ be a $C$-Galois extension of $\mathcal{E}$ and let $(\mathcal{D}, C, \psi)$ be the canonical entwining structure associated to it. Suppose there exists a convolution invertible collection $\Phi=\left\{\Phi_{X Y}: C \longrightarrow \operatorname{Hom}_{\mathcal{D}}(X, Y)\right\}_{X, Y \in \mathrm{Ob}(\mathcal{D})}$ of right $C$-comodule maps. Then, the categories $\mathscr{M}(\psi)_{\mathcal{D}}^{C}$ and $\operatorname{Mod}-\mathcal{E}$ are equivalent.

Proof. We consider the collection $\left\{\operatorname{id}_{X 0} \otimes \operatorname{id}_{X 1} \in \operatorname{Hom}_{\mathcal{D}}(X, X) \otimes C\right\}_{X \in \mathrm{Ob}(\mathcal{E})}$ which is group-like for the coring $h \otimes C$ with respect to $\mathcal{E}$. We define

$$
\begin{gathered}
\mathscr{F}: \operatorname{Mod}-\mathcal{E} \longrightarrow \mathscr{M}(\psi)_{\mathcal{D}}^{C}, \quad \mathcal{M} \mapsto \mathcal{M} \otimes_{\mathcal{E}} h, \\
\mathscr{G}: \mathscr{M}(\psi)_{\mathcal{D}}^{C} \longrightarrow \operatorname{Mod}-\mathcal{E}, \quad \mathcal{N} \mapsto \mathcal{N}^{c o(h \otimes C)}
\end{gathered}
$$

Using Lemma 4.19 and Proposition 4.17, we see that the functors $\mathscr{F}$ and $\mathscr{G}$ are well-defined. We now verify that $\mathscr{G}_{\circ} \mathscr{F} \cong \operatorname{id}_{\operatorname{Mod}-\mathcal{E}}$ i.e., $(\mathcal{M} \otimes \mathcal{E} h)^{\operatorname{co}(h \otimes C)} \cong \mathcal{M}$ for any $\mathcal{M} \in \operatorname{Mod}-\mathcal{E}$.

From Lemma 4.10, we know that $h \otimes C \cong h \otimes_{\mathcal{E}} h$ as $\mathcal{D}$-corings. Under this

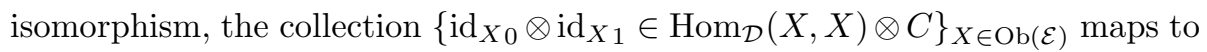
the collection $\left\{\operatorname{id}_{X} \otimes \operatorname{id}_{X} \in \mathbf{h}_{X} \otimes_{X} \mathbf{h}\right\}_{X \in \mathrm{Ob}(\mathcal{E})}$ which is group-like for $h \otimes_{\mathcal{E}} h$ with respect to $\mathcal{E}$. Therefore, it suffices to show that $\mathcal{M} \cong\left(\mathcal{M} \otimes_{\mathcal{E}} h\right)^{\operatorname{co}\left(h \otimes_{\mathcal{E}} h\right)}$. 
By Lemma (4.20)(iii), we have an inclusion $i: \mathcal{M} \longrightarrow \mathcal{M} \otimes_{\mathcal{E}} h$ of right $\mathcal{E}$-modules. It is clear that

$$
i(M) \subseteq\left(\mathcal{M} \otimes_{\mathcal{E}} h\right)^{c o\left(h \otimes_{\mathcal{E}} h\right)} .
$$

By definition, $\tilde{\rho}=\rho_{\mathcal{M} \otimes_{\mathcal{E}} h}: \mathcal{M} \otimes_{\mathcal{E}} h \longrightarrow\left(\mathcal{M} \otimes_{\mathcal{E}} h\right) \otimes_{\mathcal{D}}\left(h \otimes_{\mathcal{E}} h\right)$ is determined by

$$
\tilde{\rho}(X)(m \otimes f)=m \otimes_{\mathcal{E}} \operatorname{id}_{Y} \otimes_{\mathcal{E}} f \quad \forall m \otimes f \in \mathcal{M}(Y) \otimes_{X} \mathbf{h}(Y)
$$

for each $X \in \mathrm{Ob}(\mathcal{D})$. The coinvariants $\left(\mathcal{M} \otimes_{\mathcal{E}} h\right)^{c o\left(h \otimes_{\mathcal{E}} h\right)}: \mathcal{E}^{o p} \longrightarrow \operatorname{Vect}_{K}$ are given by

$$
\begin{aligned}
& \left(\mathcal{M} \otimes_{\mathcal{E}} h\right)^{c o\left(h \otimes_{\mathcal{E}} h\right)}(X) \\
& =\left\{\sum_{Y \in \mathrm{Ob}(\mathcal{E})} m_{Y} \otimes f_{Y} \in \mathcal{M} \otimes_{X} \mathbf{h} \mid \tilde{\rho}(X)\left(\sum m_{Y} \otimes f_{Y}\right)=\sum m_{Y} \otimes_{\mathcal{E}} f_{Y} \otimes_{\mathcal{E}} \operatorname{id}_{X}\right\} .
\end{aligned}
$$

For $\sum m_{Y} \otimes f_{Y} \in\left(\mathcal{M} \otimes_{\mathcal{E}} h\right)^{c o(h \otimes \mathcal{E} h)}(X)$, we now have

$$
\begin{aligned}
\tilde{\rho}(X)\left(\sum m_{Y} \otimes f_{Y}\right) & =\sum m_{Y} \otimes_{\mathcal{E}} f_{Y} \otimes_{\mathcal{E}} \operatorname{id}_{X} \\
& =\sum m_{Y} \otimes_{\mathcal{E}} \operatorname{id}_{Y} \otimes_{\mathcal{E}} f_{Y} \in\left(\mathcal{M} \otimes_{\mathcal{E}} h\right) \otimes_{\mathcal{E} X} \mathbf{h} .
\end{aligned}
$$

We set $\mathcal{P}:=\left(\mathcal{M} \otimes_{\mathcal{E}} h\right) / \mathcal{M} \in \operatorname{Mod}-\mathcal{E}$ and consider the following short exact sequence:

$$
0 \longrightarrow \mathcal{M} \stackrel{i}{\longrightarrow} \mathcal{M} \otimes_{\mathcal{E}} h \stackrel{\eta}{\longrightarrow} \mathcal{P} \longrightarrow 0 .
$$

Then $\eta$ induces the morphism $\eta \otimes \operatorname{id}_{h}:\left(\mathcal{M} \otimes_{\mathcal{E}} h\right) \otimes_{\mathcal{E}} h \longrightarrow \mathcal{P} \otimes_{\mathcal{E}} h$ of right $\mathcal{E}$-modules which, for each $X \in \mathrm{Ob}(\mathcal{D})$, is given by

$$
\begin{aligned}
\left(\eta \otimes \operatorname{id}_{h}\right)(X):\left(\mathcal{M} \otimes_{\mathcal{E}} h\right) \otimes_{\mathcal{E} X} \mathbf{h} \longrightarrow \mathcal{P} \otimes_{\mathcal{E} X} \mathbf{h}, & \\
m^{\prime} \otimes f^{\prime} \otimes g^{\prime} & \mapsto \eta(Y)\left(m^{\prime} \otimes f^{\prime}\right) \otimes g^{\prime},
\end{aligned}
$$

where $m^{\prime} \in \mathcal{M}(Z), f^{\prime} \in \operatorname{Hom}_{\mathcal{D}}(Y, Z), g^{\prime} \in \operatorname{Hom}_{\mathcal{D}}(X, Y)$ and $Y, Z \in \operatorname{Ob}(\mathcal{E})$. Applying $\left(\eta \otimes \mathrm{id}_{h}\right)(X)$ to $(4.22)$, we obtain

$$
\begin{aligned}
\sum \eta(X)\left(m_{Y} \otimes_{\mathcal{E}} f_{Y}\right) \otimes_{\mathcal{E}} \operatorname{id}_{X} & =\sum \eta(Y)\left(m_{Y} \otimes_{\mathcal{E}} \operatorname{id}_{Y}\right) \otimes_{\mathcal{E}} f_{Y} \\
& =\sum \eta(Y)\left(i(Y)\left(m_{Y}\right)\right) \otimes_{\mathcal{E}} f_{Y}=0 .
\end{aligned}
$$

Applying Lemma (4.20)(iii) to the inclusion $\mathcal{P} \hookrightarrow \mathcal{P} \otimes_{\mathcal{E}} h$, it follows from (4.23) that $\sum \eta(X)\left(m_{Y} \otimes_{\mathcal{E}} f_{Y}\right)=0$ for every $X \in \operatorname{Ob}(\mathcal{E})$. Therefore,

$$
\sum m_{Y} \otimes f_{Y} \in i(\mathcal{M})(X) \text {. }
$$

This proves that $\mathcal{M} \cong\left(\mathcal{M} \otimes_{\mathcal{E}} h\right)^{c o\left(h \otimes_{\mathcal{E}} h\right)}$. 


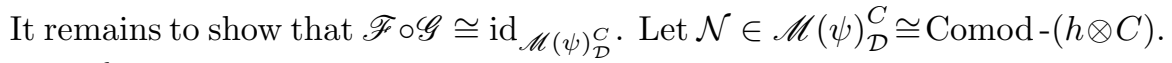
Then, $\mathcal{N}$ is a right $\mathcal{D}$-module with a given morphism

$$
\rho_{\mathcal{N}}: \mathcal{N} \longrightarrow \mathcal{N} \otimes_{\mathcal{D}}(h \otimes C) \cong \mathcal{N} \otimes_{\mathcal{D}}\left(h \otimes_{\mathcal{E}} h\right) \cong \mathcal{N} \otimes_{\mathcal{E}} h
$$

in $\mathscr{M}(\psi)_{\mathcal{D}}^{C}$. By definition, $\mathcal{N}^{c o(h \otimes C)}$ is the equalizer of the following morphisms:

$$
0 \longrightarrow \mathcal{N}^{\operatorname{co}(h \otimes C)} \longrightarrow \mathcal{N} \underset{j}{\stackrel{\rho_{\mathcal{N}}}{\longrightarrow}} \mathcal{N} \otimes_{\mathcal{E}} h
$$

where $j$ is given by

$$
j(X): \mathcal{N}(X) \longrightarrow \mathcal{N} \otimes_{\mathcal{E} X} \mathbf{h}, \quad n \mapsto n \otimes \mathrm{id}_{X},
$$

for every $X \in \operatorname{Ob}(\mathcal{D})$. By Lemma $4.20(\mathrm{i})$, it follows that $\mathcal{N}^{c o(h \otimes C)} \otimes_{\mathcal{E} X} \mathbf{h}$ is the equalizer of the following morphisms:

$$
0 \longrightarrow \mathcal{N}^{c o(h \otimes C)} \otimes_{\mathcal{E} X} \mathbf{h} \longrightarrow \mathcal{N} \otimes_{\mathcal{E} X} \mathbf{h} \underset{j \otimes \text { id }}{\stackrel{\rho_{\mathcal{N}} \otimes \mathrm{id}}{\longrightarrow}} \mathcal{N} \otimes_{\mathcal{E}} h \otimes_{\mathcal{E} X} \mathbf{h}
$$

Comparing with (4.9), we observe that $j \otimes \mathrm{id}=\operatorname{id}_{\mathcal{N}} \otimes_{\mathcal{E}} \Delta_{h \otimes_{\mathcal{E}} h}(X,-)$. Using the coassociativity of $\rho_{\mathcal{N}}: \mathcal{N} \longrightarrow \mathcal{N} \otimes_{\mathcal{E}} h$, it follows from (4.25) that $\rho_{\mathcal{N}}(X)$ factorises through $\mathcal{N}^{c o(h \otimes C)} \otimes_{\mathcal{E} X} \mathbf{h}$, which is denoted by

$$
\rho_{\mathcal{N}}^{\prime}(X): \mathcal{N}(X) \longrightarrow \mathcal{N}^{c o(h \otimes C)} \otimes_{\mathcal{E} X} \mathbf{h} \subseteq \mathcal{N} \otimes_{\mathcal{E} X} \mathbf{h} .
$$

We claim that $\rho_{\mathcal{N}}^{\prime}: \mathcal{N} \longrightarrow \mathcal{N}^{c o(h \otimes C)} \otimes_{\mathcal{E}} h$ is an isomorphism in $\mathscr{M}(\psi)_{\mathcal{D}}^{C}$. From the counit property, we know that $\left(\operatorname{id}_{\mathcal{N}} \otimes_{\mathcal{D}} \varepsilon_{h \otimes_{\mathcal{E}} h}\right) \circ \rho_{\mathcal{N}}=\mathrm{id}_{\mathcal{N}}$. Hence, $\rho_{\mathcal{N}}$ is a monomorphism and so is $\rho_{\mathcal{N}}^{\prime}$. It remains to show that $\rho_{\mathcal{N}}^{\prime}(X)$ is an epimorphism for each $X \in \operatorname{Ob}(\mathcal{D})$. For each $X \in \operatorname{Ob}(\mathcal{D})$, we define

$$
\begin{aligned}
\zeta(X): \mathcal{N}^{c o(h \otimes C)} \otimes_{\mathcal{E} X} \mathbf{h} & \longrightarrow \mathcal{N}(X), \\
\sum_{Y \in \mathrm{Ob}(\mathcal{D})} n_{Y} \otimes f_{Y} & \mapsto \sum_{Y \in \mathrm{Ob}(\mathcal{D})} \mathcal{N}\left(f_{Y}\right)\left(n_{Y}\right) .
\end{aligned}
$$

Since $\rho_{\mathcal{N}}^{\prime}$ is a morphism of right $\mathcal{D}$-modules, we now have

$$
\begin{aligned}
\rho_{\mathcal{N}}^{\prime}(X)\left(\zeta(X)\left(n_{Y} \otimes f_{Y}\right)\right) & =\rho_{\mathcal{N}}^{\prime}(X)\left(\mathcal{N}\left(f_{Y}\right)\left(n_{Y}\right)\right) \\
& =\left(\mathcal{N}^{c o(h \otimes C)} \otimes_{\mathcal{E}} h\right)\left(f_{Y}\right)\left(\rho_{\mathcal{N}}^{\prime}(Y)\left(n_{Y}\right)\right) \\
& =\left(\mathcal{N}^{\operatorname{co}(h \otimes C)} \otimes_{\mathcal{E}} h\right)\left(f_{Y}\right)\left(n_{Y} \otimes \operatorname{id}_{Y}\right)=n_{Y} \otimes f_{Y} .
\end{aligned}
$$

This shows that $\mathscr{F} \circ \mathscr{G} \cong \mathrm{id}{\mathscr{M}(\psi)_{\mathcal{D}}^{C}}_{\text {. }}$ 


\section{References}

[1] J. Y. Abuhlail, Dual entwining structures and dual entwined modules, Algebras and Representation Theory 8 (2005), 275-295.

[2] M. Balodi, A. Banerjee and S. Ray, Cohomology of modules over $H$-categories and co$H$-categories, Canadian Journal of Mathematics 72 (2020), 1352-1385.

[3] T. Brzeziński, On modules associated to coalgebra Galois extensions, Journal of Algebra 215 (1999), 290-317.

[4] T. Brzeziński, Frobenius properties and Maschke-type theorems for entwined modules, Proceedings of the American Mathematical Society 128 (2000), 2261-2270.

[5] T. Brzeziński, The structure of corings: induction functors, Maschke-type theorem, and Frobenius and Galois-type properties, Algebras and Representation Theory 5 (2002), $389-410$.

[6] T. Brzeziński and P. M. Hajac, Coalgebra extensions and algebra coextensions of Galois type, Communications in Algebra 27 (1999), 1347-1367.

[7] T. Brzeziński, and S. Majid, Coalgebra bundles, Communications in Mathematical Physics 191 (1998), 467-492.

[8] T. Brzeziński, S. Caenepeel, G. Militaru and S. Zhu, Frobenius and Maschke type theorems for Doi-Hopf modules and entwined modules revisited: a unified approach, in Ring Theory and Algebraic Geometry (León, 1999), Lecture Notes in Pure and Applied Mathematics, Vol. 221, Dekker, New York, 2001, pp. 1-31.

[9] D. Bulacu, S. Caenepeel and B. Torrecillas, Frobenius and separable functors for the category of entwined modules over cowreaths, I: General theory, Algebrs and Representation Theory 23 (2020), 1119-1157.

[10] D. Bulacu, S. Caenepeel and B. Torrecillas, Frobenius and separable functors for the category of entwined modules over cowreaths, II: applications, Journal of Algebra 515 (2018), 236-277.

[11] S. Caenepeel, Galois corings from the descent theory point of view, in Galois Theory, Hopf Algebras, and Semiabelian Categories, Fields Institute Communications, Vol. 43, American Mathematical Society, Providence, RI, 2004, pp. 163-186.

[12] S. Caenepeel and E. De Groot, Modules over weak entwining structures, in New trends in Hopf algebra theory (La Falda, 1999), Contemporary Mathematics, Vol. 267, American Mathematical Society, Providence, RI, 2000, pp. 31-54.

[13] S. Caenepeel, G. Militaru, B. Ion and S. Zhu, Separable functors for the category of Doi-Hopf modules, applications, Advances in Mathematics 145 (1999), 239-290.

[14] S. Caenepeel, G. Militaru and S. Zhu, A Maschke type theorem for Doi-Hopf modules and applications, Journal of Algebra 187 (1997), 388-412.

[15] S. Caenepeel, G. Militaru and S. Zhu, Doi-Hopf modules, Yetter-Drinfel'd modules and Frobenius type properties, Transactions of the American Mathematical Society 349 (1997), 4311-4342.

[16] C. Cibils and A. Solotar, Galois coverings, Morita equivalence and smash extensions of categories over a field, Documenta Mathematica 11 (2006), 143-159.

[17] S. Dăscălescu, C. Năstăsescu and Ş. Raianu, Hopf Algebras, Monographs and Textbooks in Pure and Applied Mathematics, Vol. 235, Marcel Dekker, New York, 2001.

[18] Y. Doi, Unifying Hopf modules, Journal of Algebra 153 (1992), 373-385. 
[19] S. Estrada and S. Virili, Cartesian modules over representations of small categories, Advances in Mathematics 310 (2017), 557-609.

[20] A. Grothendieck, Sur quelques points d'algèbre homologique, Tôhoku Mathematical Journal 9 (1957), 119-221.

[21] L. Jia, The sovereign structure on categories of entwined modules, Journal of Pure and Applied Algebra 221 (2017), 867-874.

[22] B. Mitchell, Rings with several objects, Advances in Mathematics 8 (1972), 1-161.

[23] B. Mitchell, Some applications of module theory to functor categories, Bulletin of the American Mathematical Society 84 (1978), 867-885.

[24] D. Mumford and J. Fogarty, Geometric Invariant Theory, Ergebnisse der Mathematik und ihrer Grenzgebiete, Vol. 34, Springer, Berlin, 1982.

[25] C. Năstăsescu, M. Van den Bergh and F. Van Oystaeyen, Separable functors applied to graded rings, Journal of Algebra 123 (1989), 397-413.

[26] M. D. Rafael, Separable functors revisited, Communications in Algebra 18 (1990), 14451459.

[27] P. Schauenburg, Doi-Koppinen Hopf modules versus entwined modules, New York Journal of Mathematics 6 (2000), 325-329.

[28] H.-J. Schneider, Principal homogeneous spaces for arbitrary Hopf algebras, Israel Journal of Mathematics 72 (1990), 167-195.

[29] B. Stenström, Rings of Quotients, Springer, New York-Heidelberg, 1975.

[30] M. Takeuchi, A correspondence between Hopf ideals and sub-Hopf algebras, Manuscripta Mathematica 7 (1972), 251-270. 